\title{
THE CHEBYSHEV POLYNOMIAL METHOD OF ITERATION
}

JANUARY 1967

CONTRACT AT-11-1-GEN-14

BETTIS ATOMIC POWER LABORATORY, PITTSBURGH, PA., OPERATED FOR THE U. S. ATOMIC ENERGY COMMISSION BY WESTINGHOUSE ELECTRIC CORPORATION 


\section{DISCLAIMER}

This report was prepared as an account of work sponsored by an agency of the United States Government. Neither the United States Government nor any agency Thereof, nor any of their employees, makes any warranty, express or implied, or assumes any legal liability or responsibility for the accuracy, completeness, or usefulness of any information, apparatus, product, or process disclosed, or represents that its use would not infringe privately owned rights. Reference herein to any specific commercial product, process, or service by trade name, trademark, manufacturer, or otherwise does not necessarily constitute or imply its endorsement, recommendation, or favoring by the United States Government or any agency thereof. The views and opinions of authors expressed herein do not necessarily state or reflect those of the United States Government or any agency thereof. 


\section{DISCLAIMER}

Portions of this document may be illegible in electronic image products. Images are produced from the best available original document. 
UC-32: Mathematics and Computers

Special Distribution

CFSTI PRICES

THE CHEBYSHEV POLYNOMIAL METHOD OF ITERATION

L. A. Hageman

H. C. $\$ 2.00: \mathrm{MN} \cdot 65$

This document is PUBLICLY RELWASABLE

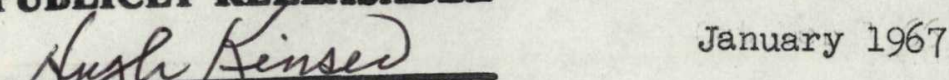

Authotizing Oficial

Date: $10-1-12$

TUCLEAF SCIENCE ABSTRACIS

CONTRACT AT-11-1-GEN-14

For sale by the Clearinghouse for Federal Scientific and Technical Information, National Bureau of Standards, U.S. Department of Commerce, Springfield, Virginia.

This document is an interim memorandum prepared primarily for internal reference and does not represent a final expression of the opinion of Westinghouse. When this memorandum is distributed externally, it is with the express understanding that Westinghouse makes no representation as to completeness, accuracy, or usability of information contained therein.

\section{LEGAL NOTICE}

This report was prepared as an account of Government sponsored work. Neither the United States, nor the Commission, nor any person acting on behalf of the Commission: A. Makes any warranty or representation, expressed or implied, with respect to the accu-
acy, completeness, or usefulness of the information contained in this report, or that the use racy, completeness, or usefulness of the information contained in this report, or that the use
of any information, apparatus, method, or process disclosed in this report may not infringe privately owned rights; or

B. Assumes any liabilities with respect to the use of, or for damages resulting from the use of any information, apparatus, method, or process disclosed in this report.

As used in the above, "person acting on behalf of the Commission" includes any employee or contractor of the Commission, or empioyee of such contractor, to the extent that such employee or contractor of the Commission, or employee of such contractor prepares, disseminates, or provides access to, any information pursuant to his employment or contract with the Commission, or his employment with such contractor. 
This report was prepared as an account of Government sponsored work. Neither the United States, nor the Commission, nor any person acting on behalf of the Commission:

A. Makes any warranty or representation, expressed or implied, with respect to the accuracy, completeness, or usefulness of the information contained in this report, or that the use of any information, apparatus, method, or process disclosed in this report may not infringe privately owned rights; or

B. Assume any liabilities with respect to the use of, or for damages resulting from the use of any information, apparatus, method, or process disclosed in this report.

As used in the above, "person acting on behalf of the Commission" includes any employe or contractor of the Commission, or employe of such contractor, to the extent that such employe or contractor of the Commission, or employe of such contractor, prepares, disseminates, or provides access to any information pursuant to his employment or contract with the Commission, or his employment with such contractor. 
I. INTRODUCTION

II. THE CHEBYSHEV POLYNOMIAL METHOD , 3

1. Introduction

2. The Power Method

3. The Chebysher Polynomial Method

4. Complex Eigenvalues

A. Complex Eigenvalues and the Real Domain 13 Chebysher Polynomial

B. The Complex Domain Chebysher Polynomial 17

5. Rates of Convergence 19

6. An Incomplete Set of Eigenvectors 25

A. Principal Vectors $\quad 25$

B. The Power Method 30

C. The Chebysher Polynomial Method 32

D. Principal Vectors of Grade Greater than Two 36

III. THE ESTTMATION OF THE DOMTNANCE RATIO d AND THE TERMINATION 40 OF THE ITERATIVE PROCESS

1. The Estimation of $a$ and $b \quad 40$

2. Chebyshew Strategy 45

3. Terminating the Iterative Procedure 49

IV. NYMERICAL EXAMPLES

APPENDIX A: The Real Domain Chebysher Polynomial and 84 Complex Eigenvalue

APPENDIX B: The Inhomogeneous Problem 90

1. The Cyclic Chebysher Method 94

$\begin{array}{ll}\text { REFERENCES } & 98\end{array}$

$\begin{array}{ll}\text { ACKNOWLEDGEMENT } & 99\end{array}$ 
In this report the prectical use of the Chebysher polynomial method of iteration is discussed. The convergence behavior of the Chebyshev method is given and a numerical strategy is described which can be used to estimate the required acceleration parameters. Numerical examples are given and discussed.

\section{TBE CHEBYSHEV POLYNOMIAL METHOD OF IRERAMION}

\section{A. Hageman}

\section{INTRODUCTION}

If the eigenvalues $\left\{\sigma_{i}\right\}_{i=1}^{i=n}$ or a real nxn matrix $G$ are ordered such that $\left|\sigma_{n}\right| \leq\left|\sigma_{n-1}\right| \leq \ldots \leq\left|\sigma_{2}\right| \leq\left|\sigma_{1}\right|$, then $\sigma_{1}$ is called the dominant eigenvalue of $G$ if $\left|\sigma_{1}\right|>\left|\sigma_{2}\right|$. Many practical problems in applied mathematics require knowledge of this dominant eigenvalue and its associated eigenvector.

A standara iterative method for finding the dominant eigenvalue and its associated eigenvector is the well-known power method. For any matrix $G$ with a dominant eigenvalue, the power method is a convergent process provided, of course, that the iniciel guess vector has a nonzero component of the dominant eigenvector. However, when the dominance ratio $\bar{\sigma} \equiv\left|\sigma_{2}\right| /\left|\sigma_{1}\right|$, of the matrix $G$ is close to unity, the rate of convergence of the power method is very slow. Whus, one would like to sint ways to excelerate the convergence rate of the basic power me"hod.

One such accelergtion scheme is the Chebyshew polynomial extrapolation method. The improvement achieved by chebyshev polynomial extrapolation depends strongly on the properties of the eigenvalues and elgentedtors of the matrix G. Normally, in applying Chebyshev polynomials, it is sssumed that the eigenwectors of $G$ span the assoclated rector space $V_{n}(c)$ of $q$ and that the eigenvalues of G are rea?. Often, however, Chebyshev extrapolation improwes the rate of convergence even though the eigenvalues are not real and/or the 
eigenvectors do not span the vector space. For this case, though, the acceleration achieved may be small.

The convergence rate of the power method is uniquely determined by the properties of the matrix $G$ and the initial guess yector; whereas, the convergence rate of the Chebysher extrapolation method also depends on the choice of three parameters. The optimum parameter values, i.e., those values for the parameters which maximize the rate of convergence, are functions of the domain in the complex plane which contains the eigenvalues of $G$. Generally, the eigenvalue domain, and hence also the optimum parameters, is not known a priori. Thus, estimating the optimum parameter values is an important but often neglected froblem in the practical application of the Chebysher extrapolation method.

The purpose of this report is to discuss the practical use of the Chebyshev polynomial method of iteration. First, we define the method and give the well-known convergence properties of the Chebysher iteration method assuming that the eigenvectors span the associated vector space and that the eigenvalues are real. We then discuss the convergence behavior of the Chebysher method when these assumptions on the eigenvalues and eigenvectors are relaxed. Practical numerical means by which to estimate the needed parameters are described and a numerical strategy given. Finally, numerical examples are given and discussed.

Although this report is concerned primarily with the solution of the homogeneous eigenvalue problem, much of what is said is valid also for the inhomogeneous problem. The use of the Chebyshev polynomial method of iteration in the solution of the inhomogeneous matrix problem is discussed briefly in Appendix B。 
II. THE CHEBYSHEV POLYNOMIAL METHOD

1. Introduction

Let $G$ be a real nxn matrix with eigenvalues $\left\{\sigma_{i}\right\}_{i=1}^{i=n}$ and eigenvectors $\left\{\underline{x}_{i}\right\}_{i=1}^{i=n}$. We assume that the matrix $G$ has a dominant eigenvalue which is positive and that, the eigenvalues of $\mathrm{G}$ are ordered such that

$$
\left|\sigma_{n}\right| \leq\left|\sigma_{n-1}\right| \leq \cdots \leq\left|\sigma_{3}\right| \leq\left|\sigma_{2}\right|<\sigma_{1} .
$$

We let $x_{i}$ be the eigenvector associated with the eigenvalue $\sigma_{i}$, i。e., $\mathrm{x}_{\mathrm{i}}=\sigma_{i} \underline{x}_{i}$ 。

Unless the contrary is explicitly stated, we also assume that $\sigma_{2}$ is real and positive and that $\sigma_{2}>\left|\sigma_{i}\right|$ for $i \geq 3$.

In this chapter, we are concerned with the problem of solving the homogeneous equation

$$
\underline{G x}=\sigma \underline{X}
$$

for the dominant eigenvalue $\sigma_{I}$ and its corresponding eigenvector $x_{I}$.

\section{Whe Fower Method}

One may iteratively solve the eigenvalue problem (2.1) using the well-known power methok. Given the real initial vector $x(0)$ and eigenvalue $o(0)$, the power method generates successive estimates for the eigenvector $\underline{x}_{1}$ and eigenvalue $\sigma_{1}$ by the process

$$
\begin{aligned}
& \mathbb{E}(k)=\frac{G}{\mathbb{C}^{(k-1)}} \underline{x}(k-1) \\
& \sigma(k)=\sigma(k-1) \frac{[\underline{v}(k), \underline{v}(k)]}{[\underline{\underline{v}(k), \underline{x}(k-1)]}} \\
& \underline{x}(k)=\underline{v}(k),
\end{aligned}
$$


where $[\underline{r}, \underline{s}]$ denotes the scalar product of the vector $\underline{r}$ with the vector $\underline{s}$,

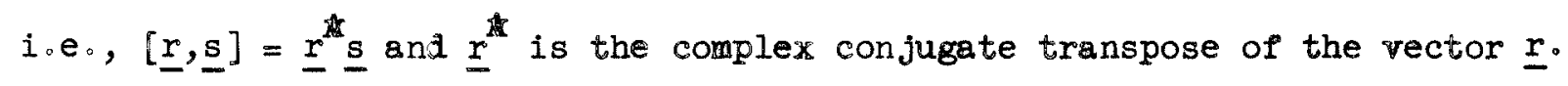
The integer $\mathrm{k}$ in $(2.2)$ is the iteration index number.

There are many ways by which the eigenvalue may be estimated in the power method. The eigenvalue estimate $\sigma(k)$ in $(2.2)$ is obtained by the socalled modified Rayleigh quotient [Bilodeau and Hageman (1957)]。 other techniques which may be used to estimate the eigenvalue are the Rayleigh quotient, the component sum, and the single component techniques. Unless the matrix $G$ is symmetric ${ }^{I}$, it usually makes very little difference which technique is used to estimate the eigenvalue.

For the power method of iteration, the eigenvector is more crucial and more evasive than the eigenvalue. Intuitively, this may be seen by considering the eigenvalue problem (2.1)。Given the eigenvalue $\sigma_{1}$, it is still a difficult, task to determine $\underline{x}_{1}$; whereas, given the eigenvector $\underline{x}_{1}$, it is easy to calculate $\sigma_{1}$. Thus, for the most part, we shall concentrate on the convergence of the eigenvector.

For any iterative process, the answers to several questions must be considered. For example,

1. Does the iterative process converge?

2. If the process is convergent, how fast or at what rate does it converge?

3. What practical criterion may be used to terminate the iterative process?

In this chapter, we shall be concerned with answers to the first two questions. The third question will be discussed in a later chapter.

If the matrix $G$ is symetric, the Rayleigh quotient and the modified Rayleigh quotient have certain adwantages over the other techniques. 
Since the matrix $G$ has a dominant eigenvalue, it follows [Faddeev and Faddeeva (1963) ] that the power iterative method (2.2) is convergent, 1.e.,

$$
\lim _{k \rightarrow \infty} \sigma(k)=\sigma_{1} \text { and } \lim _{k \rightarrow \infty} x(k)=\underline{x}_{1}
$$

The rate of convergence of the power method depends primarily on how well separated the dominant eigenvalue $\sigma_{1}$ is from the other eigenvalues of $G$. To see why this is true, let us first assume ${ }^{l}$ that the eigenvectors of $G$ span $v_{n}(c)$. Thus, the eigenpector estimate $x\left(k_{1}\right)$ after $k_{1}$ iterations may be written as

$$
\underline{x}\left(k_{1}\right)=\underline{x}_{1}+\sum_{i=2}^{n} c_{i} \underline{x}_{i}
$$

where the $c_{i}$ are scalars. whe corresponding error vector $\underline{E}\left(k_{1}\right)$ for iteration $k_{1}$ can be expressed by

$$
E\left(k_{1}\right) \equiv x_{1}\left(k_{1}\right)-\underline{x}_{1}=\sum_{i=2}^{n} c_{i-i}
$$

For iteration $\left(k_{1}+1\right)$, we have

$$
\underline{x}\left(k_{1}+1\right)=\frac{G}{\sigma\left(k_{1}\right)} \underline{x}\left(k_{1}\right)=\frac{\sigma_{1}}{\sigma\left(k_{1}\right)} x_{1}+\sum_{i=2}^{n}\left(\frac{\sigma_{i}}{\sigma\left(k_{1}\right)}\right) c_{i} x_{i} .
$$

If we now assume that $k_{1}$ is large enough so that the eigenvalue estimates $\sigma\left(k_{1}+r\right), r \geq 0$, are sufficiently close to $\sigma_{1}$, then for iteration $\left(k_{1}+r\right)$, we have

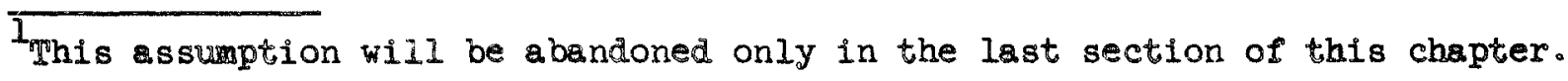




$$
\underline{x}\left(k_{1}+r\right) \approx\left(\frac{G}{\sigma_{1}}\right)^{r} \underline{x}\left(k_{1}\right)=\underline{x}_{1}+\sum_{i=2}^{n}\left(\frac{\sigma_{i}}{\sigma_{1}}\right)^{r} c_{i} \underline{x}_{i}
$$

and

$$
E\left(k_{1}+r\right) \approx \sum_{i=2}^{n}\left(\frac{\sigma_{i}}{\sigma_{1}}\right)^{r} c_{i=1}
$$

Thus, the rate at which the error vector $E(k)$ approsches the null vector or equivalently the rate at which $\underline{x}(k)$ approaches $\underline{x}_{1}$ depends on how well separated the dominant eigenvalue $\sigma_{1}$ is from the other eigenvalues of $G$.

If the dominance ratio $d$ of the matrix $G$ is defined by

$$
\mathrm{d} \equiv \max _{i \neq 1} \frac{\left|\sigma_{i}\right|}{\left|\sigma_{1}\right|}=\frac{\left|\sigma_{2}\right|}{\left|\sigma_{1}\right|}
$$

then the most slowly decaying contribution to the error vector is multiplied by a scalar of modulus d each iteration. Thus, $d$ may be taken as the average reduction factor per iteration for successive error vectors. We define the average rate of convergence $R$ for the power method as

$$
\mathrm{R} \equiv-\ln \mathrm{d}
$$

Roughly speaking, the reciprocal of $R$ is a measure of the number of iterations required to reduce the initial error vector by a factor $e$, where $e$ is the base of the natural logarithms. Thus, a natural criterion for the comparison of different iterative methods is the size of their respective rates of convergence. For a more detaịled discussion on convergence rates, see varga (1962), page 62 . 
In the next section we shall describe the Chebyshev polynomial extrapolation method which often may be used to accelerate the convergence rate of the basic power method.

\section{The Chebyshey Polynomial Extrapolation Method}

From Eq. (2.5) we see that the performance of $r$ power iterations results in the most slowly decaying contribution to the error vector being multiplied by a factor of $d^{r}$. We note that these $r$ power iterations correspond to applying the marrix operstor $\left(\frac{G}{\sigma_{1}}\right)^{r}$ to the vector $\underline{x}\left(k_{1}\right)$. Now if a $r$-th degree matrix polynomial $a_{r}\left(\frac{G}{\sigma_{1}}\right)$ were used to operate on $\underline{x}\left(k_{1}\right)$, we could express ${ }^{2}$ $\underline{x}\left(k_{1}+r\right)$ as

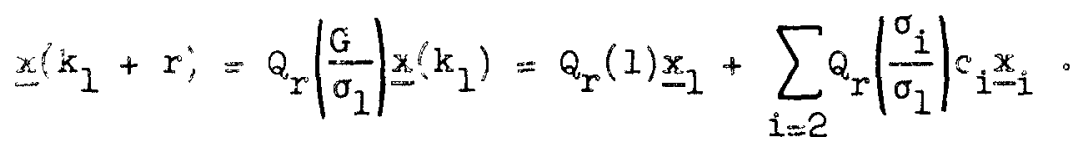

Hence, if we could choose the polynomial $Q_{r}(y)$ such that $Q_{r}(1)=1$ and $\sum_{i=2} Q_{r}\left(\frac{\sigma_{i}}{\sigma_{l}}\right) c_{i} \underline{x}_{i}=\underline{0}$, then we would have $\underline{x}\left(k_{1}+r\right)=\underline{x}_{1}$. Even if such a polynomial existed, it woula be a sunction of the $c_{i}$, $x_{i}$, and $c_{1}$, which generally are not known for all i. "Therefore, such a special polynomial is usually out of the question.

Suppose, however, that the eigenvalues $\sigma_{i}$ of $G$ are real and satisfy $\mathrm{b} \leq \sigma_{i} / \sigma_{1} \leq \mathrm{a}$ for $i \geq 2.3$ then we can try to choose for $Q_{r}(y)$ that polynomial $P_{r}(y)$ having the leas msximum modulus over the range $b \leq y \leq d$ and such that

If $Q_{r}(y)=\sum_{k=0}^{r} b_{k} y^{k}$ is of polynomial of degree $r$ in $y$, then the matrix polynomial $Q_{r}(B)$ in the matrix $B$ is defined as $Q_{r}(B)=\sum_{k=0}^{r} b_{k} B^{k}$.

We are still assuming that the eigenvectors $x_{i}$ of $G \operatorname{span} v_{n}(C)$. In keeping with the assumption made in section 1 of this chapter, we assume that $d>\mid b !$. 
$P_{r}(1)=1$. Such a polynomial exists [Flanders and Shortley (1950)] and can be given explicitly in terms of Chebysher polynomials by

$$
P_{r}(y) \equiv \frac{T_{r}\left(\frac{2 y-d-b}{d-b}\right)}{T_{r}\left(\frac{2-d-b}{d-b}\right)}
$$

where $T_{r}(w)$ is the Chebysher polynomial of degree $r$. For $r>0$, $\mathbb{T}_{r}(w) \equiv \cos \left[r \cos ^{-1} w\right]$ if $|w| \leq 1$ and $T_{r}(w) \equiv \cosh \left[r \cosh ^{-1} w\right]$ if $|w| \geq 10^{1}$ With $Q_{r}(y)=P_{r}(y)$, the polynomial method of (2.9) is called the Chebyshev polynomial method and the matrix $\left(\frac{G}{\sigma_{I}}\right)$ is called the argument matrix. The well known recurrence relation for Chebysher polynomials

$$
T_{r}(w)=2 w T_{r-1}(w)-T_{r-2}(w), r \geq 2,
$$

where $T_{0}(w)=1$ and $T_{1}(w)=w$, enables one to successively generate the polynomials $P_{r}(y)$ in a straightforward way [see, for example, Hageman (1963) p. 27]. Starting with $\underline{x}\left(k_{1}\right)$, one may generate successively

$$
\begin{gathered}
\underline{x}\left(k_{1}+1\right)=P_{1}\left(\frac{G}{\sigma_{1}}\right) \underline{x}\left(k_{1}\right) \\
\underline{x}\left(k_{1}+2\right)=P_{2}\left(\frac{G}{\sigma_{1}}\right) \underline{x}\left(k_{1}\right) \\
\vdots \\
\underline{x}\left(k_{1}+r\right)=P_{r}\left(\frac{G}{\sigma_{1}}\right) \underline{x}\left(k_{1}\right)
\end{gathered}
$$
${ }_{\text {If }} w$ is any complex number, then $T_{r}(w)$ may be expressed [Forsythe and Wasow
$(1960)$ ] as

$$
T_{r}(w) \equiv 1 / 2\left[\left(w+\sqrt{w^{2}-1}\right)^{r}+\left(w-\sqrt{w^{2}-1}\right)^{r}\right] \text {. }
$$


using the procedure

$$
\left\{\begin{aligned}
\underline{v}\left(k_{1}+t\right)= & \frac{G}{\sigma\left(k_{1}+t-1\right)} \underline{x}\left(k_{1}+t-1\right) \\
\underline{x}\left(k_{1}+t\right)=\underline{x}\left(k_{1}+t-1\right) & +\alpha_{k_{1}+t}\left[\underline{v}\left(k_{1}+t\right)-\underline{x}\left(k_{1}+t-1\right)\right] \\
& +\beta_{k_{1}+t}\left[\underline{x}\left(k_{1}+t-1\right)-\underline{x}\left(k_{1}+t-2\right)\right] \\
\sigma\left(k_{1}+t\right)=\sigma\left(k_{1}+t-1\right) & \frac{\left[\underline{v}\left(k_{1}+t\right), \underline{v}\left(k_{1}+t\right)\right]}{\left[\underline{v}\left(k_{1}+t\right), \underline{x}\left(k_{1}+t-1\right)\right]}
\end{aligned}\right.
$$

for $t=1,2,3, \ldots$ (The $\sigma$ calculation is included in (2.12) to take into account the fact that $\sigma\left(k_{1}\right)$ is not exactly equal to $\left.\sigma_{1} \cdot\right) \alpha_{k_{1}+t}$ and $\beta_{k_{1}+t}$ are functions of $d, b$, and $t$ and are given by

$$
\left\{\begin{array}{l}
\alpha_{k_{1}+1}=\frac{2}{2-d-b} ; \beta_{k_{1}+1}=0 \text { and for } t \geq 2 \\
\alpha_{k_{1}+t}=\frac{4}{d-b} \frac{T_{t-1}\left(\frac{2-d-b}{d-b}\right)}{T_{t}\left(\frac{2-d-b}{d-b}\right)} ; \beta_{k_{1}+t}=\frac{T_{t-2}\left(\frac{2-d-b}{d-b}\right)}{T_{t}\left(\frac{2-d-b}{d-b}\right)}
\end{array}\right.
$$

Since

$$
\max _{\substack{b \leq y \leq d}}\left|P_{r}(y)\right|=P_{r}(d)=\frac{1}{\left.T_{r} \mid \frac{2-d-b}{d-b}\right)},
$$

we see that the most slowly decaying contributions to the error vector are multiplied by a factor of modulus $P_{r}(d)$ in $r$ iterations. For d close to unity, the Chebyshev polynomial method of iteration is an order of magnitude faster than the power method. Table 2.1 shows as a function of $d$ the gain in speed of convergence one may obtain using the Chebyshev polynomial method of iteration compared to the power method. For Table 2.1, b is assumed to be zero. 


\begin{tabular}{|c|c|c|c|c|c|c|c|c|}
\hline \multirow{2}{*}{$d$} & \multicolumn{2}{|c|}{1 ITERATION } & \multicolumn{2}{|c|}{3 ITERATIONS } & \multicolumn{2}{|c|}{5 ITERATIONS } & \multicolumn{2}{|c|}{10 ITERATIONS } \\
\cline { 2 - 9 } & $(d)^{1}$ & $P_{1}(d)$ & $(d)^{3}$ & $P_{3}(d)$ & $(d)^{5}$ & $P_{5}(d)$ & $(d)$ & $P_{10}(d)$ \\
\hline .6 & .6 & .429 & .216 & .023 & .078 & .001 & .006 & $\ldots$ \\
\hline .7 & .7 & .538 & .343 & .049 & .168 & .004 & .028 & $\ldots$ \\
\hline .8 & .8 & .667 & .512 & .111 & .328 & .016 & .108 & $\ldots$ \\
\hline .9 & .9 & .818 & .729 & .276 & .590 & .076 & .349 & .003 \\
\hline .95 & .95 & .905 & .857 & .481 & .774 & .204 & .599 & .021 \\
\hline .97 & .97 & .942 & .913 & .624 & .859 & .337 & .737 & .060 \\
\hline .985 & .985 & .970 & .956 & .778 & .927 & .539 & .860 & .170 \\
\hline .99 & .99 & .980 & .970 & .843 & .951 & .647 & .904 & .266 \\
\hline .992 & .992 & .984 & .976 & .871 & .961 & .700 & .923 & .325 \\
\hline .995 & .995 & .990 & .985 & .917 & .975 & .792 & .951 & .457 \\
\hline .998 & .998 & .996 & .994 & .965 & .990 & .908 & .980 & .700 \\
\hline
\end{tabular}

TABLE 2.1

From $(2.14)$ we see that $\left[P_{r}(d)\right]^{l / r}$ is the average reduction factor per iteration for successive error vectors of the Chebysher polynomial method. Thus, the average rate of convergence for $r$ iterations of the Chebyshev polynomials method is defined [Varga (1962), page 134] as

$$
R_{r}\left[P_{r}\right] \equiv-\ln \left[P_{r}(d)\right]^{I / r}
$$

From Eq. (2.14), it follows that $R_{r}\left[P_{r}\right]$ increases monotonically with $r$ and that [Varga (1962), page 139] 


$$
R_{\infty}\left[P_{r}\right] \equiv \lim _{r \rightarrow \infty} R_{r}\left[P_{r}\right]=\cosh ^{-1}\left(\frac{2-d-b}{d-b}\right)
$$

In a later section, we shall discuss convergence rates in more detail.

Thus far, we have assumed that the eigenvalue bounds $d$ and $b$ are known. This, of course, is not a realistic assumption. If the estimates for $d$ and $b$ are denoted by $d_{0}$ and $b_{0}$, then we shall take $P_{r, \sigma_{0}}(y)$ to be the Chebyshev polynomial of degree $r$ in which $a_{0}$ and $b_{0}$ are used as estimates for $\mathrm{d}$ and $\mathrm{b}$. From Eq. (2.10), we see that $\mathrm{P}_{\mathrm{r}, \mathrm{d}_{0}}(\mathrm{y})$ may be written as

$$
P_{r, a_{0}}(y)=\frac{T_{r}\left(\frac{2 y-a_{0}-b_{0}}{d_{0}-b_{0}}\right)}{T_{r}\left(\frac{2 y-d_{0}-b_{0}}{a_{0}-b_{0}}\right)}
$$

and hence

$$
\max _{b \leq y \leq d}\left|P_{r, d_{0}}(y)\right|=\frac{\left.\max \left|T_{r}\right| \frac{2 y-d_{0}-b_{0}}{a_{0}-b_{0}}\right) \mid}{\left.T_{r} \mid \frac{2-a_{0}-b_{0}}{d_{0}-b_{0}}\right)} .
$$

From the min-max property of Chebyshev polynomials or by directly comparing $(2.14)$, and $(2.18)$, we have

$$
\max _{b \leq y \leq d}\left|P_{r}(y)\right| \leq \max _{b \leq y \leq d}\left|P_{r, d_{0}}(y)\right|
$$

with equality only if $\mathrm{d}_{0}=\mathrm{d}$ and $\mathrm{b}_{0}=\mathrm{b}$.

To illustrate how the effectiveness of the Chebysher polynomial method depends on the estimate of $d$, let us consider a matrix $G$ for which 
$d=.9$ and $b=0$. If the estimates for $d$ and $b$ were correct, $i_{0} e_{\circ}, a_{0}=d=.9$ and $b_{0}=b=0$, then from Table 2.1 we have $P_{5}(d)=.076$; whereas, with $d_{0}=.8$ and $b_{0}=0$, we have from (2.18) that $\max _{b \leq y \leq d}\left|P_{5, d_{0}}(y)\right|=P_{5, d_{0}}(d) \approx .25$. Thus, in 5 iterations the most slowly conver $\bar{g} \bar{n}$ g contributions to the error vector are multiplied by a factor of modulus .076 in the optimum parameter case as compared to .25 in the non-optimum parameter case. Fence, the use of non-optimum values for $\mathrm{d}$ and $\mathrm{b}$ can result in a sizable redustion in the convergence rate of the Chebyshev method of iteration. Fortunately, as we shall see in tie next chapter, practical numerical means exist for estimating these unknown constants.

In addition to the basic assumption that; $G$ has a dominant eigenvalue $\sigma_{1}$, we have assumed, thus far, that the eigenvalues of $G$ are real and that the eigenvectors of $G$ span the associated vector space of $G_{0}{ }^{1}$ In the next section we shall relax the assumption that the eigenvalues of $G$ be real.

\section{Complex Eigenvalues}

In this section we will again assume that the eigenvectors $\left\{x_{i}\right\}_{i=1}^{i=n}$ of $G$ span the vector space $V_{n}(C)$ but the assumption that all the eigenvalues of $\mathrm{G}$ are real will be relaxed. We shall assume only that the dominant eigenvalue $\sigma_{1}$ is real and positive.

We will present two approaches which, hopefully, will illustrate the effect of complex eigenvalues on the Chebyshev polynomial method of iteration. The first approach will be to show the effect of complex eigenvalues on the convergence rate when the Chebyshey polynomial of Eq. (2.10) is applied. We

\footnotetext{
We have also assumed that $\sigma_{1}$ and $\sigma_{2}$ are positive and that $\left.\sigma_{2}\right\rangle\left|\sigma_{i}\right|$ for $i \geq 3$. These assumptions, however, were made merely for reasons of simplicity and are not restricting.
} 
note that the Chebyshev polynomial given in Eq. (2.10) is based on the assumption that the eigenvalues of $G$ are real. The second approach will be to change the argument of the Chebysher polynomial so that the min-max property of these polynomials will be valid over part of the complex plane. ${ }^{1}$

\section{A. Complex Eigenvalues and the Real Domain Chebyshev Polynomial}

Let the eigenvalues of $G$ be denoted by $\left\{\sigma_{i}\right\}_{i=1}^{i=n}$. As before, we take the dominant eigenvalue $\sigma_{1}$ to be real and positive but now we assume only that the quantities $\left\{\frac{\sigma_{1}}{\sigma_{1}}\right\}_{i=2}^{i=n}$ are contained in a connected region $D$ in the complex plane. The dominance ratio is again given by $d=\left|\sigma_{2}\right| / \sigma_{1}$.

Now suppose that the Chebyshev polynomial defined by Eq. (2.10) is used in the polynomial method of (2.9). If $d_{0}$ and $b_{0}$ are used as estimates for $d$ and $b$ in (2.10) then, as in Eq. (2.17), we let

$$
P_{r, d_{0}}(z)=\frac{T_{r}\left(\frac{2 z-d_{0}-b_{0}}{d_{0}-b_{0}}\right)}{T_{r}\left(\frac{2-d_{0}-b_{0}}{d_{0}-b_{0}}\right)}
$$

Thus, from Eq. (2.9) we see that in $r$ iterations the most slowly decaying contributions to the error vector are multiplied by at most a factor of modulus $f_{r}(D)$, where

$$
f_{r}(D) \equiv \max _{z \in D}\left|P_{r, d_{0}}(z)\right|=\frac{\max \left|T_{r}\right| \frac{2 z-d_{0}-b_{0}}{d_{0}-b_{0}} \mid}{T_{r}\left(\frac{2-d_{0}-b_{0}}{a_{0}-b_{0}}\right)} .
$$

\footnotetext{
The effect of complex eigenvalues on Chebyshev extrapolation is also dis-
} cussed by Wachspress (1966) and Wrigley (1963). 
The average reduction factor per iteration is then

$$
F_{r}(D) \equiv\left[f_{r}(D)\right]^{1 / r}
$$

We now wish to determine how $F_{Y}(D)$ is affected by the region $D$.

If the eigenvalues of $G$ are real and if $d_{0}$ and $b_{0}$ satisfy $d_{0} \geq d$ and $b_{0} \leq b$, then the region $D$ may be chosen to be the closed interval $\left[b_{0}, d_{0}\right]$ and for this case we have

$$
\left\{F_{r}\left(\left[b_{0}, d_{0}\right]\right)\right\}^{r}=\max _{z \in\left[b_{0}, d_{0}\right]}\left|P_{r, d_{0}}(z)\right|=\frac{1}{T_{r}\left(\frac{2-d_{0}-b_{0}}{a_{0}-b_{0}}\right)}
$$

Without more knowledge concerning the eigenvalues of $G, F_{r}\left(\left[b_{0}, d_{0}\right]\right)$ is the smallest average reduction factor which can be achieved by the Thebyshev poly nomial method of iteration. Thus, in seeing how $F_{r}(D)$ is affected by the region $D$, we shall use $F_{r}\left(\left[b_{0}, d_{0}\right]\right)$ as the norm. In what follows, we shall denote $F_{r}\left(\left[b_{0}, d_{0}\right]\right)$ simply by $F_{r}$.

Let $I_{r}(c)$ be the set of points in the complex $z$ plane such that the inequality $\left|P_{r, d_{0}}(z)\right| \leq\left\{c F_{r}\right\}^{r}$ is satisfied, i.e.,

$$
D_{r}(c) \equiv\left\{z:\left|P_{r, d_{0}}(z)\right| \leq\{c F r\}\right.
$$

Thus, if all eigenvalues of $\left(\frac{G}{\sigma_{1}}\right)$ except unity, are contained in $D_{r}(c)$, then the average reduction factor per iteration achieved by the Chebyshev polynomial method will be less than or equal to $\mathrm{CF}_{\mathrm{r}}$. We are only interested in $c$ over the range $1 \leq c \leq 1 / F_{r}$. For if $c \geq 1 / F_{r}$, the Chebyshev method of iteration is divergent. If $c<1$, the set $D_{r}(c)$ consists of $r$ separated regions which are centered about the $r$ real zeros of the polynomial $T_{r}\left(\frac{2 z-d_{0}-b_{0}}{d_{0}-b_{0}}\right)$ and 
thus not generally of practical interest.

Obviously, if $c$ is fixed, then $D_{r}(c)$ is a function of $r$. If $r=1$, the region $D_{1}(c)$ consists of all points on or interior to a circle with center at $\left(\frac{a_{0}+b_{0}}{2}, 0\right)$ and radius $c\left(\frac{d_{0}-b_{0}}{2}\right)$. See Figure 2.1 .

The region $D_{2}(c)$ consists of all points on or interior to the ovals of Cassini. A proof of this together with the region $\mathrm{D}_{2}(\mathrm{c})$ for an arbitrary $c$ is given in Appendix $A$. The regions $D_{2}(c)$ for $c=1$ and $c=1 / F_{2}$ are given in Figure 2.2. Thus, if we choose to cyclically apply ${ }^{1}$ the polynomial $P_{2, d_{0}}(z)$ and if all the eigenvalues of $\left(\frac{G}{\sigma_{1}}\right)$ except unity, were contained in $\mathrm{D}_{2}(\mathrm{c})$, then the average reduction factor per iteration would be no greater than $\mathrm{CF}_{2}$.

In the limit as $r$ approaches infinity, the region $D_{\infty}(c)$ consists of all points on or interior to the ellipse

$$
\frac{\left[x-\left(\frac{a_{0}+b_{0}}{2}\right)\right]^{2}}{\left[\left(\frac{a_{0}-b_{0}}{4}\right)\left(c+\frac{1}{c}\right)\right]^{2}}+\frac{y^{2}}{\left[\left(\frac{a_{0}-b_{0}}{4}\right)\left(c-\frac{1}{c}\right)\right]^{2}}=1 .
$$

A proof of this is given in Appendix A. The regions $D_{\infty}(c)$ for $c=1$ and ${ }^{2}$ $c=1 / F_{\infty}$ are given in Figure 2.3 . Note that $D_{\infty}(1)$ is simply the line segment $b_{0}<z<a_{0}$

We now shall give a more basic approach to the complex eigenvalue problem.

$\overline{I n s t e a d}$ of letting $r$ tend to infinity.

$2^{2}$ The limit of $F_{r}$ as $r \rightarrow \infty$ can be expressed as

$$
F_{\infty}=\frac{d_{0}-b_{0}}{2-\left(d_{0}+b_{0}\right)+2 \sqrt{\left(1-d_{0}\right)\left(1-b_{0}\right)}} .
$$

This will be discussed in more detail in section 5 of this chapter. 


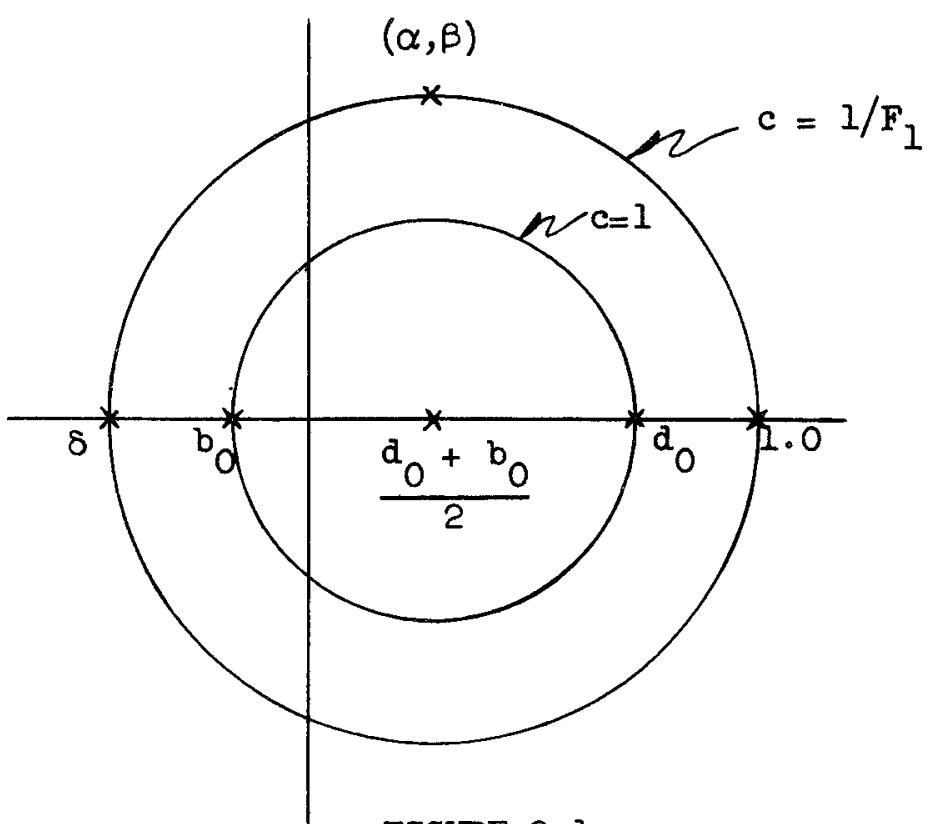

FIGURE 2.1

$D_{1}$ Region

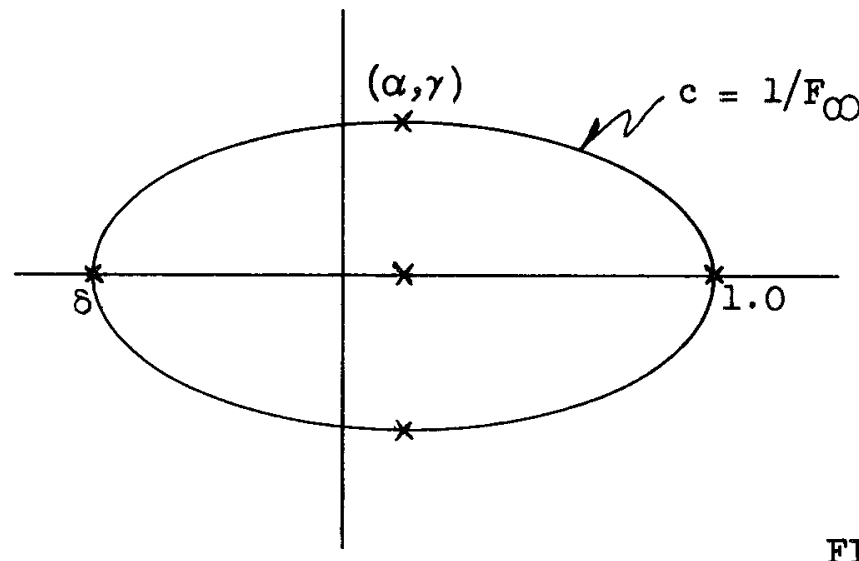

FIGURE 2.3

$\mathrm{D}_{\infty}$ Region

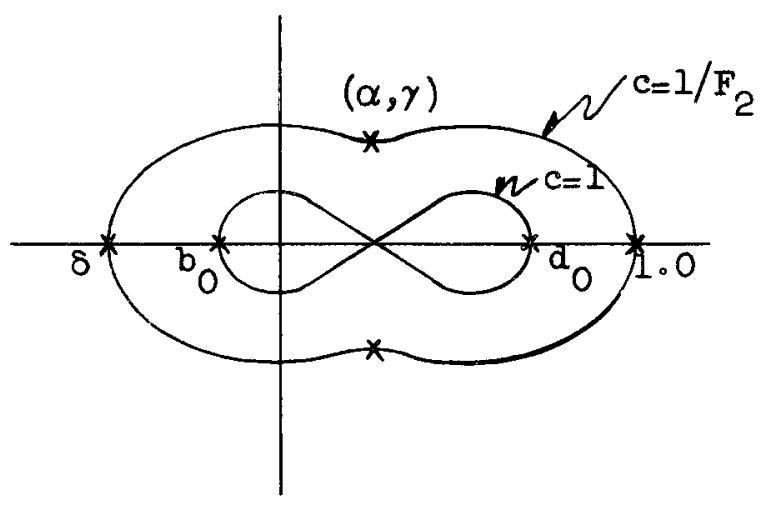

FIGURE 2.2

$\mathrm{D}_{2}$ Region

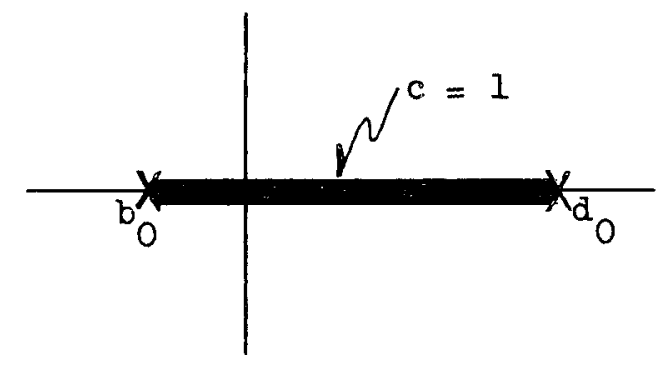
$\alpha=\frac{d_{0}+b_{0}}{2}$
$\gamma=\sqrt{\left(1-d_{0}\right)\left(1-b_{0}\right)}$
$\beta=1-\left(\frac{a_{0}+b_{0}}{2}\right)$
$\delta=-1+\left(a_{0}+b_{0}\right)$ 


\section{B. The Complex Domain Chebyshev Polynomial}

Suppose now that the quantities $\left\{\sigma_{i} / \sigma_{1}\right\}_{i=2}^{i=n}$ are contained in the ellipse (see Figure 2.4)

$$
\frac{\left[x-\left(\frac{a+b}{2}\right)\right]^{2}}{\left(\frac{d-b}{2}\right)^{2}}+\frac{y^{2}}{\epsilon^{2}}=1,
$$

where $0<\epsilon<\frac{d-b}{2}$. Thus, the real eigenvalue premise used in obtaining

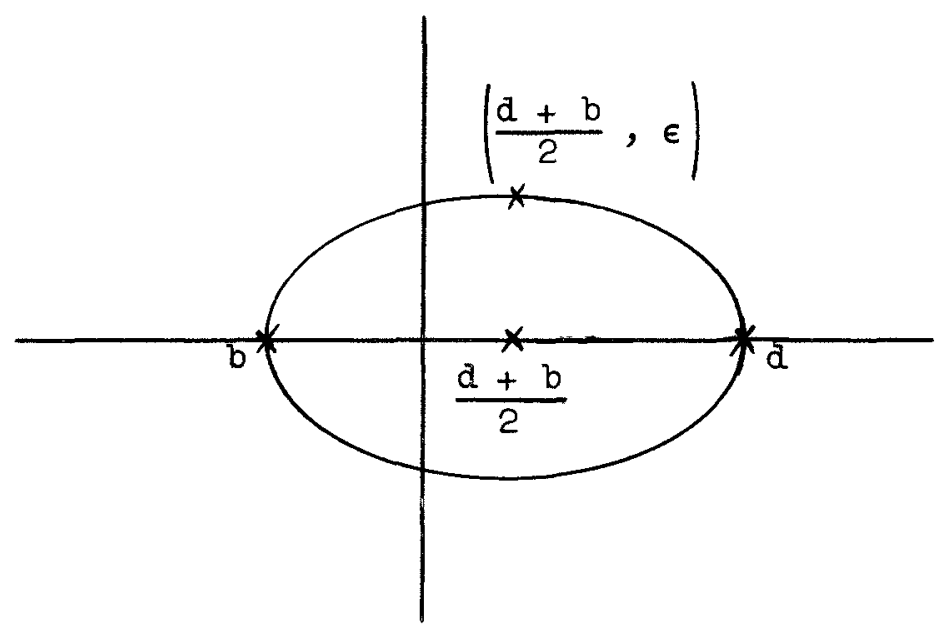

Figure 2.4

the polynomial (2.10) is not valid. For the complex case one would like to choose for $Q_{r}(y)$ in $E q$. (2.9) that polynomial $\widetilde{P}_{r}(y)$ having the least maximum modulus over the ellipse (2.25) and its interior. Clayton (1963) has shown that such a polynomial exists and that it is unique, real, and can be expressed in terms of Chebyshev polynomials by 


$$
\tilde{P}_{r}(y) \equiv \frac{T_{r}\left(\frac{2 y-d-b}{\left[(d-b)^{2}-4 \epsilon^{2}\right]^{1 / 2}}\right)}{T_{r}\left(\frac{2-a-b}{\left[(d-b)^{2}-4 \epsilon^{2}\right]^{1 / 2}}\right)}
$$

where the $T_{r}(w)$ are again Chebysher polynomials of degree $r$. When $\epsilon=0$, $\tilde{P}_{r}(y)$ reduces to $P_{r}(y)$. We shall refer to the polynomial method of (2.9) as the complex Chebysher polynomial method when $Q_{r}(y)=\tilde{P}_{r}(y)$.

The vectors $\underline{x}\left(k_{1}+r\right)=\widetilde{P}_{r}\left(\frac{G}{\sigma_{1}}\right) \underline{x}\left(k_{1}\right)$ for the complex Chebysher polynomial method may be generated successively using the same procedure (2.12) as described for the real Chebyshev case. For the complex case, however, the parameters $\alpha$ and $\beta$ are given by

$$
\alpha_{k_{1}+1}=\frac{2}{2-d-b} ; \beta_{k_{1}+1}=0 \text { and for } t \geq 2
$$

$\alpha_{k_{1}+t}=\frac{4}{\left[(d-b)^{2}-4 \epsilon^{2}\right]^{1 / 2}} \frac{T_{t-1}\left(\frac{2-d-b}{\left[(d-b)^{2}-4 \epsilon^{2}\right]^{1 / 2}}\right)}{T_{t}\left(\frac{2-d-b}{\left[(d-b)^{2}-4 \epsilon^{2}\right]^{1 / 2}}\right)} ; \beta_{k_{1}+t}=\frac{T_{t-2}\left(\frac{2-d-b}{\left[(d-b)^{2}-4 \epsilon^{2}\right]^{1 / 2}}\right)}{T_{r}\left(\frac{2-d-b}{\left[(d-b)^{2}-4 \epsilon^{2}\right]^{1 / 2}}\right)}$.

If we let $D$ denote the ellipse (2.25) and its interior, then one may easily show that

$$
\max _{z \in D}\left|\tilde{P}_{r}(z)\right|=\tilde{P}_{r}(d)=\frac{T_{r}\left|\frac{d-b}{\left[(d-b)^{2}-4 \epsilon^{2}\right]^{1 / 2}}\right|}{T_{r}\left(\frac{2-d-b}{\left[(a-b)^{2}-4 \epsilon^{2}\right]^{1 / 2}}\right)}
$$

so that $\left[\tilde{\mathrm{P}}_{r}(\mathrm{~d})\right]^{1 / \mathrm{r}}$ is the average reduction factor per iteration for successive error vectors of the complex Chebyshev polynomial method. 
As before, we define the average rate of convergence for $r$ iterations of the complex Chebyshev method to be

$$
R_{r}\left[\tilde{P}_{r}\right] \equiv-\ln \left[\tilde{P}_{r}(d)\right]^{I / r}
$$

From Eq. (2.27) one may show that $R_{r}\left[\tilde{P}_{r}\right]$ increases monotonically with $r$ and that

(2.29) $R_{\infty}\left[\tilde{P}_{r}\right) \equiv \lim _{r \rightarrow \infty} R_{r}\left[\tilde{P}_{r}\right]=\cosh ^{-1}\left(\frac{2-d-b}{\left[(d-b)^{2}-4 \epsilon^{2}\right]^{1 / 2}}\right)-\cosh ^{-1}\left(\frac{d-b}{\left[(d-b)^{2}-4 \epsilon^{2}\right]^{1 / 2}}\right)$

Since $\widetilde{P}_{r}(y)$ belongs to the set of polynomials from which $P_{r}(y)$ was chosen, we must have that $P_{r}(d) \leq \tilde{P}_{r}(d)$ and hence $R_{r}\left[\tilde{P}_{r}\right] \leq R_{r}\left[P_{r}\right]$ with strict inequality for all $r \geq 2$. Thus, the complex Chebyshev polynomial method does not achieve as great an improvement over the straight power method as does the real Chebysher polynomial method. In the next section we will compare the quantities $R, R_{\infty}\left[P_{r}\right]$, and $R_{\infty}\left[\tilde{P}_{r}\right]$ when $d$ is close to unity.

\section{Rates of Convergence}

As given previously, the average rate of convergence for the complex Chebyshev polynomial method increases monotonically with $\mathbf{r}$ to the limit $R_{\infty}\left[\tilde{\mathrm{P}}_{\mathrm{r}}\right]$, where

$$
R_{\infty}\left[\tilde{P}_{r}\right]=\cosh ^{-1}\left(\frac{2-a-b}{\left[(a-b)^{2}-4 \epsilon^{2}\right]^{1 / 2}}\right)-\cosh ^{-1}\left(\frac{a-b}{\left[(d-b)^{2}-4 \epsilon^{2}\right]^{1 / 2}}\right)
$$

Since $\cosh ^{-1}(y)=\ln \left[y+\sqrt{\left.y^{2}-1\right]}\right.$, we have

$$
R_{\infty}\left[\tilde{P}_{r}\right]=\ln \left\{\frac{(2-a-b)+\left[(2-d-b)^{2}-(a-b)^{2}+4 \epsilon^{2}\right]^{1 / 2}}{d-b+2 \epsilon}\right\}
$$


or equivalently

$$
R_{\infty}\left[\tilde{F}_{r}\right]=-\ln \left\{\frac{d-b+2 \varepsilon}{2-d-b+2\left[(1-d)(1-b)+\epsilon^{2}\right]^{1 / 2}}\right\} .
$$

Similarly, for the real Chebyshev polynomial method we have

$$
R_{\infty}\left[P_{r}\right]=-\ln \left\{\frac{d-b}{2-a-b+2[(1-d)(1-b)]^{1 / 2}}\right\} .
$$

We recall that the quantities $d, b$, and $e$ are assumed to satisfy

$$
\mathrm{d}<1,|\mathrm{~b}|<\mathrm{d} \text {, and } \epsilon<\frac{\mathrm{d}-\mathrm{b}}{2} \text {. }
$$

The average rate of convergence for the power method does not depend on $r$ and from Eq. (2.8) is given by

$$
R=-\ell n d
$$

We will now compare these convergence rates when d is near unity or equivalently when $\delta$, where

$$
8=1-a,
$$

is near zero. Since $-\ln y=(1-y)+\frac{(1-y)^{2}}{2}+\frac{(1-y)^{3}}{3}+\ldots$ for $0<y \leq 1$, we may write $\mathrm{Eq} \cdot(2.33)$ as

$$
R=8+\frac{\delta^{2}}{2}+\frac{\delta^{3}}{3}+\cdots
$$


Thus, for small 8, a good approximation for the rate of convergence of the power method is

Similarly, $R_{\infty}\left[P_{r}\right]$ may be expressed as

$$
R_{\infty}\left[P_{r}\right]=\delta\left[P_{r}\right]+\frac{\left\{\delta\left[P_{r}\right]\right\}^{2}}{3}+\frac{\left\{\delta\left[P_{r}\right]\right\}^{3}}{3}+\cdots
$$

where

$$
\delta\left[P_{r}\right]=1-\frac{d-b}{2-d-b+2[(1-d)(1-b)]^{1 / 2}}=2\left[\frac{\delta+[\delta(1-b)]^{1 / 2}}{\delta+(1-b)+2[\delta(1-b)]^{1 / 2}}\right] .
$$

Since $I-b>0$, we may write $\delta\left[P_{r}\right]$ as

$$
\delta\left[\mathrm{P}_{r}\right]=\frac{2 \sqrt{\frac{\delta}{1-b}}+2\left(\frac{\delta}{1-b}\right)}{1+2 \sqrt{\frac{\delta}{1-b}}+\frac{\delta}{1-b}} \text {. }
$$

In most practical applications $b$ will range from 0 to $-d$ so that $I-b$ usually varies from 1 to 2 . Thus, for small $\delta$ it is reasonable to assume that $\delta /(1-b)$ is also small. Hence, for small $\delta$, a good approximation for the rate of convergence of the real Chebyshev polynomial method is

$$
R_{\infty}\left[P_{r}\right] \approx 2 \sqrt{\frac{\delta}{1-b}}
$$

Note that as $b$ varies from 0 to $-d, R_{\infty}\left[P_{r}\right]$ varies only from $2 \sqrt{\delta}$ to $\sqrt{2} \cdot \sqrt{\delta}$. Thus $R_{\infty}\left[P_{r}\right]$ is not greatly affected by the value of $b$. 
For the complex Chebyshey polynomial, $R_{\infty}\left[\tilde{\mathrm{P}}_{\mathrm{r}}\right]$ inay be expressed as

$$
R_{\infty}\left[\tilde{P}_{r}\right]=\delta\left[\tilde{P}_{r}\right]+\frac{\left\{\delta\left[\tilde{P}_{r}\right]\right\}^{2}}{2}+\frac{\left\{\delta\left[\tilde{P}_{r}\right]\right\}^{3}}{3}+\ldots,
$$

where

$\delta\left[\tilde{P}_{r}\right]=1-\frac{a-b+2 \epsilon}{2-a-b+2\left[(1-a)(1-b)+\epsilon^{2}\right]^{1 / 2}}=2\left[\frac{\delta-\epsilon+\left[\delta(1-b)+\epsilon^{2}\right]^{1 / 2}}{\delta+(1-b)+2\left[\delta(1-b)+\epsilon^{2}\right]^{1 / 2}}\right]$.

If we let $\mathrm{K}^{2} \equiv \epsilon^{2} /\left[(1-b ; \delta]\right.$, then $\delta\left[\widetilde{\mathrm{P}}_{\mathrm{r}}\right]$ may be written as

$$
\delta\left[\tilde{P}_{r}\right]=\frac{2 \sqrt{\frac{\delta}{1-b}} \sqrt{1+K^{2}}-K+2 \frac{\delta}{1-b}}{1+2 \sqrt{\frac{\delta}{1-b}} \sqrt{1+K^{2}}+\frac{\delta}{1-b}} .
$$

Now $\epsilon$ must satisfy $0 \leq \epsilon \leq \frac{a-b}{2}$ so that $K$ must satisfy $0 \leq K<\frac{1}{\sqrt{8}}\left[\frac{a-b}{2 \sqrt{1-b}}\right]$. Thus, for small $\delta$, $K$ may take on large values. For $\varepsilon=0(K=0), \delta\left[\tilde{P}_{r}\right]$ is the same as $\delta\left[\mathrm{P}_{\mathrm{r}}\right]$ and thus for small $\delta$

$$
\mathrm{R}_{\infty}\left[\tilde{\mathrm{P}}_{r}\right]_{\varepsilon=0} \approx 2 \sqrt{\frac{\delta}{1-b}}
$$

For $\varepsilon=\sqrt{\delta(1-b)}(K=1)$, we have for small $\delta$

$$
R_{\infty}\left[\tilde{P}_{r}\right]_{\varepsilon}=\sqrt{\delta(1-b)} \approx .828 \sqrt{\frac{\delta}{1-b}}
$$

Thus, as $\epsilon$ varies from 0 to only $\sqrt{\delta(l-b)}$, the rate of convergence of the complex Chebyshev method varies by more than a factor of 2 . We now shall see what happens to $R_{\infty}\left[\tilde{P}_{r}\right]$ as $\varepsilon$ approaches $\frac{d-b}{2}$ or equiwalently as $K$ approaches 
$\frac{1}{\sqrt{\delta}} \frac{a-b}{2 \sqrt{1-b}}$

With $\epsilon>0$ and using the fact that $\epsilon=K \sqrt{\delta(1-b)}, E q \cdot(2.41)$ may be written as

$$
\delta\left[\tilde{P}_{r}\right]=\frac{\delta}{\epsilon}\left\{\frac{2 K\left[\sqrt{1+K^{2}}-K\right]+2 K \sqrt{\frac{\delta}{1-b}}}{1+2 \sqrt{\frac{\delta}{1-b}} \sqrt{1+K^{2}}+\frac{\delta}{1-b}}\right\} \equiv \frac{\delta}{\epsilon}\{f(K)\} .
$$

For $K>0, f(K)$ is an increasing function ${ }^{1}$ of $K$ and $f\left(\frac{1}{\sqrt{\delta}}\left[\frac{d-b}{2 \sqrt{1-b}}\right]\right)=\frac{d-b}{1-b+\delta}$. Thus, $f(K)<I$ for $a l l o<K<\frac{1}{\sqrt{\delta}}\left[\frac{d-b}{2 \sqrt{1-b}}\right]$. Hence, for $\epsilon>0$ and small $\delta$ we have

$$
\mathrm{R}_{\infty}\left[\tilde{\mathrm{P}}_{\mathrm{r}}\right]<\frac{\delta}{\epsilon}
$$

As $\epsilon$ approaches $\frac{a-b}{2}$, we have from $(2.44)$ and the above that

$$
R_{\infty}\left[\tilde{\mathrm{P}}_{r}\right]_{\epsilon \rightarrow \frac{\mathrm{d}-\mathrm{b}}{2}} \approx \frac{\delta}{\epsilon} \frac{\mathrm{d}-\mathrm{b}}{1-\mathrm{b}+\delta} \approx \frac{2 \delta}{1-\mathrm{b}+\delta}
$$

If $b=-d$, note that $R_{\infty}\left[\tilde{P}_{r}\right]$ approaches the convergence rate of the power method as $\epsilon$ approaches d. This agrees with the well-known result [Varga (1957)] that as the ellipse containing the normalized eigenvalues $\left\{\sigma_{i} / \sigma_{1}\right\}_{i=2}^{n}$ tends to a circle, the min-max polynomial defined by (2.26) tends to $\left(y-\left.\left(\frac{a+b}{2}\right)\right|^{r}\right.$ or just $y^{r}$ when $b=-d$.

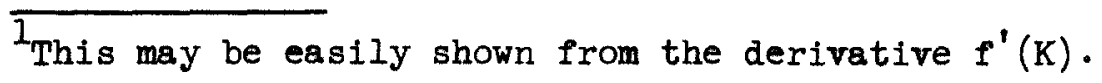


From expressions $(2.36),(2.39)$, and $(2.45)$ we have for $\delta=1$ - d

close to zero

$(2.47)$

$$
R_{\infty}\left[P_{r}\right] \approx \frac{2}{\sqrt{\delta(1-b)}} R
$$

and for $\epsilon>0$

$(2.48)$

$$
\mathrm{R}_{\infty}\left[\tilde{\mathrm{P}}_{\mathrm{r}}\right] \lesssim \frac{\mathrm{R}}{\epsilon}
$$

Table 2.2 indicates how the different convergence rates vary as a function of $d, b$, and $\epsilon$.

\begin{tabular}{|c|c|c|c|c|c|c|}
\hline \multirow{2}{*}{$\mathrm{d}$} & \multirow{3}{*}{$\mathrm{R}$} & $\mathrm{3}$ & \multicolumn{2}{|c|}{$\mathrm{R}_{\infty}\left[\mathrm{P}_{r}\right]$} & $\left.\tilde{\mathrm{P}}_{r}\right]$ \\
\cline { 3 - 7 } & $\mathrm{b}=0$ & $\mathrm{~b}=-.1$ & $\mathrm{~b}=-.3$ & $\begin{array}{l}\mathrm{b}=-.1 \\
\epsilon=.166\end{array}$ & $\begin{array}{l}\mathrm{b}=-.3 \\
\epsilon=.4\end{array}$ \\
\hline .8 & .223 & .963 & .911 & .829 & .621 & .386 \\
.9 & .104 & .654 & .622 & .569 & .376 & .213 \\
.99 & .051 & .455 & .433 & .397 & .225 & .114 \\
.995 & .010 & .200 & .191 & .176 & .055 & .024 \\
& .002 & .146 & .136 & .125 & .012 & .005 \\
\hline
\end{tabular}

TABLE 2.2

VARIATION IN CONVERGENCE RATES WITH $\mathrm{d}, \mathrm{b}$, AND $\epsilon$ 
Thus, when the eigenvalues are real and d close to unity, the real Chebyshev polynomial method is an order of magnitude faster than the power method. For the complex eigenvalue case, the complex Chebyshev polynomial method is likely to achieve a much smaller, though still welcome, increase in convergence rate over the power method.

We remark that the comparisons given above are based on $R_{\infty}$ and not $R_{r}$. As mentioned previously, $R_{r}$ increases monotonically with $r$ and is bounded by $R_{1} \leq R_{r} \leq R_{\infty}$. For $r=1$ and for $d$ close to unity we have

$$
\left\{\begin{array}{l}
\mathrm{R}_{1}\left[\mathrm{P}_{1}\right] \approx \frac{2}{1-\mathrm{b}+\delta} \mathrm{R} \text { and } \\
\mathrm{R}_{1}\left[\tilde{\mathrm{P}}_{1}\right] \approx \mathrm{R}_{1}\left[\mathrm{P}_{1}\right] .
\end{array}\right.
$$

In the next section we will discuss the case when the eigenvectors do not span the associated vector space of $G$.

\section{An Incomplete Set of Eigenvectors}

In the previous material we have assumed that the set of elgenvectors of $G$ spans the associated vector space of $G$. In this section we abandon this requirement. Before proceeding, we first give a preliminary discussion on the concept of principal vectors.

\section{A. Principal Vectors}

As used in this report, a vector is simply an ordered collection of $\mathrm{n}$ complex numbers. The totality of all such vectors with $n$ elements or components is called the n-dimensional vector space over the complex number field and is denoted by $V_{n}(C)$. Since the nxn matrix G with complex elements operating on a 
vector $\underline{x}$ in $v_{n}(C)$ merely transforms $\underline{x}$ into another vector $\underline{y}$ in $v_{n}(C)$, we say that $V_{n}(C)$ is the vector space associated with the matrix $A$.

The set of vectors $\left\{\underline{y}_{i}\right\}_{i=1}^{i=t}$ are said to span the vector space $v_{n}(C)$ if every vector in $v_{n}(C)$ can be written as a linear combination of $\underline{y}_{i}$. If the $\operatorname{set}\left\{\underline{y}_{i}\right\}_{i=1}^{i=t}$ spans $v_{n}(c)$, then [Perlis (1952)] $t \geq n$ and the set $\left\{\underline{y}_{i}\right\}_{i=1}^{i=t}$ contains precisely $n$ linearly independent vectors, $i_{0} e_{0}$, any set of $n+1$ vectors from $\left\{\underline{y}_{i}\right\}_{i=1}^{i=t}$ is dependent.

If the set of $n$ vectors $\left\{\underline{y}_{i}\right\}_{i=1}^{i=n}$ is Iinearly independent, then this set spans $v_{n}(C)$ and is said to form a basis for $v_{n}(c)$. Thus, any set of $n$ linearly independent vectors forms a basis for $V_{n}(C)$ and hence also spans $V_{n}(c)$. We now wish to define a basis for $v_{n}(c)$ in terms of the eigenvectors and principal vectors of the matrix $G$ 。

An $n \times n$ matrix $G$ with complex elements has precisely $n$ eigenvalues associated with 1t. These eigenvalues are defined to be the $\mathrm{n}$ roots of the characteristic equation ${ }^{1}$

$$
|G-z I|=z^{n}+h_{n-1} z^{n-1}+\cdots+h_{1} z+h_{0}=0
$$

The roots of (2.50) are not necessarily distinct. The number of roots to (2.50) which have the same value is called the multiplicity of that root or eigenvalue. For what follows, let the eigenvalues of $G$ be denoted by

$$
\left(\sigma_{1}\right)_{m_{1}},\left(\sigma_{2}\right)_{m_{2}}, \ldots,\left(\sigma_{i}\right)_{m_{i}}, \ldots,\left(\sigma_{t}\right)_{m_{t}},
$$

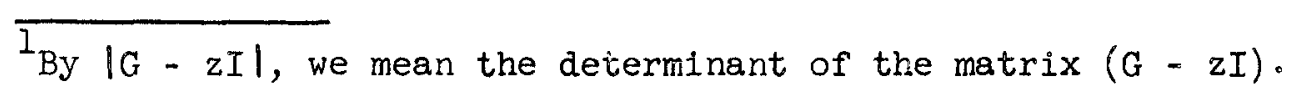


where $\sigma_{1}, \sigma_{2}, \ldots, \sigma_{t}$ are all distinct and $m_{i}$ is the multiplicity of the eigenvalue $\sigma_{i}$ and where $\sum_{i=1}^{t} m_{i}=n$.

With each eigenvalue $\sigma_{1}$ of $G$ we may associate at least one nonzero vector $\underline{x}_{i}$ which satisfies the homogeneous equation

$$
G \underline{x}_{i}=\sigma_{i} \underline{x}_{i}
$$

or equivalently

$$
\left(G-\sigma_{i} I\right) \underline{x}_{i}=\underline{0} .
$$

The existence of at least one nonzero vector $\underline{x}_{i}$ is assured since $|G-\lambda I|=0$. Any nonzero vector which satisfies $(2.52)$ is called an eigenvector of G corresponding to the eigenvalue $\sigma_{1}$. From the set of eigenvectors for $G$, we would like to pick $n$ linearly independent vectors to form a besis for $v_{n}(C)$. But, as we shall see, this is not always possible.

If the matrix G is normal ${ }^{1}$, then it is known [Perlis (1952)] that it is possible to find a basis for $V_{n}(C)$ consisting of eigenvectors of $G$. (In fact, one may choose the vectors of this basis to be mutually orthogonal.) If the matrix $G$ is not normal, it may not be possible ${ }^{2}$ to find a basis for $v_{n}(C)$ from the set of eigenvectors of $G$. However, it is always possible to find a basis for $V_{n}(C)$ from the set of principal vectors of $G$.

The matrix $G$ is normal if $G^{k} G=G G^{*}$, where $G$ is the conjugate transpose of $G$. Note that all Hermitian, skew-Hermitian, real symmetric, and real skew matrices are normal.

${ }^{2}$ One need only consider the matrix $\left(\begin{array}{l}01 \\ 00\end{array}\right)$ to show this. 
Any nonzero vector $\underline{y}_{i}$ which satisfies

$$
\left(G-\sigma_{i} I\right)^{p_{y_{i}}}=\underline{0}
$$

but for which

$$
\left(G-\sigma_{i} I\right)^{p-1} \underline{y}_{i} \neq \underline{0}
$$

is called [Householder (1953), page 32] a principal vector of grade $p$ corresponding to the eigenvalue $\sigma_{\dot{1}}$. Note that the set of eigenvectors is included in the set of principal vectors since eigenvectors are principal rectors of grade 1 .

The following theorem is a restatement of results given in sections 57 and 58 of a book by Halmos (1957).

Theorem 2.1: For each of the distinct eigenvalues $\sigma_{i}$ of (2.51), there exists positive integers $\mathrm{q}, \mathrm{p}_{1}, \mathrm{p}_{2}, \ldots, \mathrm{p}$ and nonzero vectors $\underline{\mathrm{y}}_{1}, \underline{\mathrm{y}}_{2}, \ldots, \underline{\mathrm{y}}_{\mathrm{q}}$ such that the $m_{i}$ vectors

$$
\left\{\begin{array}{c}
\underline{y}_{1},\left(G-\sigma_{i} I\right) \underline{y}_{1}, \ldots,\left(G-\sigma_{i} I\right)^{p_{1}-I} \underline{y}_{I} \\
\underline{y}_{2},\left(G-\sigma_{i} I\right) \underline{y}_{2}, \ldots,\left(G-\sigma_{i} I\right)^{p_{2}-I} \underline{y}_{2} \\
\vdots \\
\vdots \\
\underline{y}_{q},\left(G-\sigma_{i} I\right) \underline{y}_{q}, \ldots,\left(G-\sigma_{i} I\right)^{p_{q}-I} \underline{y}_{q}
\end{array}\right.
$$

are linearly independent. Moreover,

$$
\left(G-\sigma_{i} I\right)^{p_{1}} \underline{y}_{1},=\left(G-\sigma_{i} I\right)^{p} \underline{y}_{2}=\ldots=\left(G-\sigma_{i} I\right)^{p} \underline{q}_{q_{q}}=0
$$


and $p_{1}+p_{2}+\cdots+p_{q}=m_{i}$, where $m_{i}$ is defined in (2.5l).

It is easily seen that the $m_{i}$ vectors given by (2.56) are principal

vectors of $G$ corresponding to $\sigma_{i}$ and that the $q$ vectors $\left(G-\sigma_{i} I\right)^{p_{j}^{-I}} \underline{y}_{j}$, $j=1, \ldots, q$, are eigenvectors of $G$ corresponding to $\sigma_{i}$. If for each distinct eigenvalue $\sigma_{i}$ we let $Y_{i}$ denote the set of $m_{i}$ vectors given by $(2.56)$, then [Halmos (1957), pg. 113] the set of $n$ vectors $\left\{Y_{i}\right\}_{i=1}^{i=t}$ are linearly independent. Hence, for any matrix $G$, the set of principal vectors must include a basis for $v_{n}(C)$.

The integers $\mathrm{q}, \mathrm{p}_{1}, \mathrm{p}_{2}, \ldots, \mathrm{p}_{\mathrm{q}}$ of $\mathrm{Thm} .2 .1$ may also be given in terms of the elementary divisors of $G$. Corresponding to the eigenvalue $\sigma_{i}$, the matrix $G$ has the $q$ elementary divisors

$$
\left(z-\sigma_{i}\right)^{p_{1}},\left(z-\sigma_{i}\right)^{p_{2}}, \ldots,\left(z-\sigma_{i}\right)^{p_{q}} .
$$

Thus, the $p_{j}{ }^{\prime} s, j=1, \ldots, q$, are simply the degrees of the elementary divisors associated with the eigenvalue $\sigma_{i}$. If all the elementary divisors of $G$ are linear, then the principal vectors of $G$ are also eigenvectors and thus, for this case, the set of eigenvectors includes a basis for $v_{n}(C)$.

For simplicity reasons, we shall limit ourselves mainly to a discussion of the simplest case which illustrates the character of the changes that occur when the matrix $G$ has nonlinear elementary divisors. Suppose that the matrix $G$ has only one nonlinear elementary divisor and that this nonlinear divisor is of degree 2 and is associated with the eigenvalue $\sigma_{s}$. As a basis for $v_{n}(C)$, we shall use

$$
\underline{x}_{1}, \underline{x}_{-2}, \cdots, x_{s}, \underline{y}_{-1} \underline{x}_{s+1}, \cdots, \underline{x}_{n-1},
$$


where the vectors $x_{i}$ are eigenvectors of $G$ with corresponding eigenvalue $\sigma_{i}$ and satisfy $\mathrm{Gx}_{i}=\sigma_{i} \underline{x}_{i}$. The vector $\underline{y}$ is a principal vector of grade 2 corresponding to $\sigma_{s}$ and satisfies

$$
\left(G-\sigma_{s} I\right) \underline{y}=\underline{x}_{s}
$$

The existence of such a basis is guaranteed by Theorem 2.1. In what follows we shall assume that the eigenvalues of $G$ are real and that $\left|\sigma_{1}\right|>\left|\sigma_{2}\right| \geq\left|\sigma_{1}\right|$ for $i \geq 3$. We now shall see how the vector $\underline{y}$ affects the convergence rates of the power and Chebysher polynomial methods of iteration.

\section{B. The Power Method}

The elgenvector estimate $\underline{x}\left(k_{1}\right)$ after performing $k_{1}$ power iterations may be expressed in terms of the basis vectors (2.58) as

$$
\underline{x}\left(k_{1}\right)=\underline{x}_{1}+\sum_{i=2} c_{i} \underline{x}_{i}+h \underline{y},
$$

where $h$ and the $c_{i}$ are scalars. If $k_{1}$ is large enough so that the eigenvalue estimates $\sigma\left(k_{1}+r\right), r \geq 0$, are sufficiently close to $\sigma_{1}$, then for iteration $\left(k_{1}+r\right)$ we have

$$
\underline{x}\left(k_{1}+r\right) \approx\left(\frac{G}{\sigma_{1}}\right)^{r} \underline{x}\left(k_{1}\right)=\underline{x}_{1}+\sum_{i=2}\left(\frac{G}{\sigma_{1}}\right)^{r} c_{i} \underline{x}_{i}+h\left(\frac{G}{\sigma_{1}}\right)^{r} \underline{y} .
$$

The corresponding error vector $\underline{E}\left(k_{1}+r\right)=\underline{x}\left(k_{1}+r\right)-\underline{x}_{1}$ may be expressed as

$$
\underline{E}\left(k_{1}+r\right) \approx \sum_{i=2} c_{i}\left(\frac{G}{\sigma_{1}}\right)^{r} \underline{x}_{i}+h\left(\frac{G}{\sigma_{1}}\right)^{r} \underline{y} .
$$


From (2.59), we have that $G \underline{y}=\sigma_{s} \underline{y}+\underline{x}_{s} \cdot$ Thus,

$$
G^{2} \underline{y}=G\left[\sigma_{s} \underline{y}+\underline{x}_{s}\right]=\left(\sigma_{s}\right)^{2} \underline{y}+2 \sigma_{s} \underline{x}_{s}
$$

and in general

$$
G^{r} \underline{y}=\left(\sigma_{s}\right)^{r} \underline{y}+r\left(\sigma_{s}\right)^{r=1} \underline{x}_{s} .
$$

Hence, $\underline{E}\left(k_{1}+r\right)$ may be expressed as

$$
\underline{E}\left(k_{1}+r\right) \approx \sum_{i=2} c_{i}\left(\frac{\sigma_{i}}{\sigma_{1}}\right)^{r} \underline{x}_{i}+h\left(\frac{\sigma_{s}}{\sigma_{1}}\right)^{r} \underline{y}+\frac{h r}{\sigma_{1}}\left(\frac{\sigma_{s}}{\sigma_{1}}\right)^{r-1} \underline{x}_{s} .
$$

Since $\lim _{r \rightarrow \infty} r \alpha^{r}=0$ if $|\alpha|<1$, we have that, $\underset{r \rightarrow \infty}{\lim _{r \rightarrow \infty}} \underline{E}\left(k_{1}+r\right)=\underline{0}$. Thus, the power method is still a convergent process when principal vectors of grade 2 are present.

The most slowly decaying basis vector in (2.64) will depend on the magnitude of $\left|\sigma_{s} / \sigma_{I}\right|$ and is likely to vary with $r$. After $r$ power iterations, the $\underline{x}_{s}$ vector has a coefficient of

$$
=s\left(\frac{\sigma_{s}}{\sigma_{1}}\right)^{r=1}\left[\frac{\sigma_{s}}{r \sigma_{1}}+\frac{h}{\sigma_{1} c_{s}}\right]
$$

as compared with a coefficient of $c_{s}$ at the beginning of these $r$ iterations. Hence, the $x_{s}$ vector has been multiplied by a factor of

$$
M_{r}=r\left(\frac{\sigma_{s}}{\sigma_{1}}\right)^{r-1}\left[\frac{\sigma_{s}}{r_{1}}+\frac{h}{\sigma_{1} c_{s}}\right]
$$


in $r$ power iterations. The multiplication factor for the other basic vectors in the error vector expansion is, as before, $\left(\sigma_{1} / \sigma_{1}\right)$. We note that the vector $x_{s}$ is being built up if the multiplication factor is greater than unity and being reduced when this factor is less than unity。

Although $\lim _{r \rightarrow \infty} M_{r^{0}}=0$, inftially $M_{r}$ may increase with $r$. For example, if $\left|\sigma_{s} / \sigma_{1}\right|=.99$ and $h / \sigma_{1} c_{s}=1.0$, then $M_{r}$ is an increesing function of $r$ for $r \leq 90$ and is greater than unity for $r \leq 640$. If the ratio $\left|\sigma_{s} / \sigma_{1}\right|$ is small, however, then $M_{r}$ goes to zero very rapialy. For example, if $\sigma_{s}=0$, then $M_{r}=0$ for $r \geq 2$. Thus, for the power method of iteration, the effect of principal vectors of grade 2 depends rather strongly on the value of the corresponding eigenvalue.

\section{The Chebysher Polynomial Method}

Suppose that the $r$-th degree real polynomial given by $(2.10)$ is applied to the eigengector estimate $\underline{x}\left(k_{1}\right)$. We may write this as

$$
\underline{x}\left(k_{1}+r\right)=P_{r}[g(G)] \underline{x}\left(k_{1}\right)
$$

where $g(z)=\frac{2 z}{\sigma_{1}(d-b)}-\frac{d+b}{d-b}$ and $P_{r}[g(z)]=\frac{\left.v_{r}[g ! z)\right]}{T_{r}\left[g\left(\sigma_{1}\right)\right]}$, and where $T_{r}(g)$ is the Chebysher polynomial of degree $r$ in $g$. We note that the notation used in (2.66) is slightly different from that used previously. We have introduced the dependent variable $g$ merely for notational ease later.

$$
\text { With the vector } \underline{x}\left(k_{l}\right) \text { given by }(2.60) \text {, the vector } \underline{x}\left(k_{1}+r\right) \text { can be }
$$
written as

$$
\underline{x}\left(k_{1}+r\right)=\underline{x}_{1}+\sum_{i=2} c_{i} P_{r}[g(G)] \underline{x}_{1}+h P_{r}[g(G)] \underline{y}
$$


and the error vector $E\left(k_{1}+r\right)$ as

$$
\underline{E}\left(k_{1}+r\right)=\sum_{i=2} c_{i} P_{r}[g(G)] \underline{x}_{i}+h P_{r}[g(G)] \underline{y}
$$

Since the $\underline{x}_{i}$ are eigenvectors, the sum $\sum_{i=2} c_{i} P_{r}[g(G)] \underline{x}_{i}$ can be written as $\sum_{i=2} c_{i} P_{r}\left[g\left(\sigma_{i}\right)\right] \underline{x}_{i}$. We now want to see what happens to the term $P_{r}[g(G)] \underline{y}$. Since $P_{F}[g(G)]$ is a polynomial of degree $r$ in $g(G)$, we may write

$$
P_{r}[g(G)]=a_{0}+a_{1}[g(G)]+a_{2}[g(G)]^{2}+\cdots+a_{r}[g(G)]^{r}
$$

Now

$$
g(G) \underline{y}=\left[\frac{2 G}{\sigma_{1}(d-b)}-\frac{d+b}{a-b}\right] \underline{y}=g\left(\sigma_{s}\right) \underline{y}+\frac{2}{\sigma_{1}(d-b)} \underline{x}_{s} .
$$

Thus,

$$
[g(G)]^{2} \underline{y}=\left[g\left(\sigma_{s}\right)\right]^{2} \underline{y}+2\left(\frac{2}{\sigma_{1}(d-b)}\right) g\left(\sigma_{s}\right) \underline{x}_{s}
$$

and in general

$$
[g(G)]^{r} \underline{y}=\left[g\left(\sigma_{s}\right)\right]^{r} \underline{y}+r\left|\frac{2}{\sigma_{1}(\tilde{\alpha}-b)}\right|\left[g\left(\sigma_{s}\right)\right]^{r-1} \underline{x}_{s} 。
$$

Since $r\left|\frac{2}{\sigma_{1}(d-b)}\right|\left[g\left(\sigma_{s} j\right]^{r-I}=\left.\frac{a[g(z)]}{d z}\right|_{z=\sigma_{s}}\right.$, we have from Eqs. (2.69) and $(2.70)$ that

$$
P_{r}[g(G)] \underline{y}=P_{r}\left[G\left(\sigma_{s} j\right] \underline{y}+\frac{d\left\{P_{r}\left[g\left(\sigma_{s}\right)\right]\right\}}{d z} \underline{x}_{s}\right. \text {. }
$$


We also have

(2.72) $\frac{d\left(P_{r}\left[g\left(\sigma_{s}\right)\right]\right\}}{d z}=\frac{\partial g}{\partial z} \frac{d\left[P_{r}(g)\right]}{d g}=\left[\frac{2}{\sigma_{1}(d-b)}\right]\left[\frac{1}{N_{r}\left[g\left(\sigma_{l}\right)\right]}\right] \frac{d\left[T_{r}(g)\right]}{d g}$.

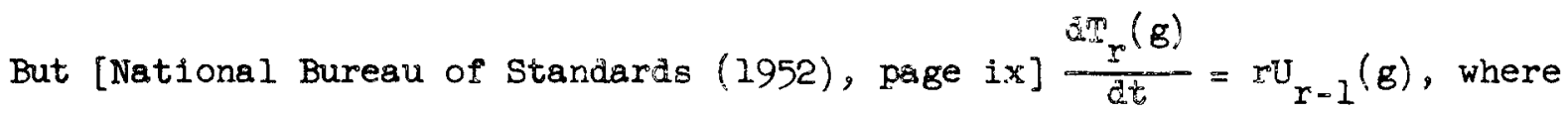
$U_{r-1}(g)$ is the Chebysher polynomial of the second kind. $U_{r-1}(g)$ is a polynomial of degree $r-1$ and is given by

$$
U_{r-1}(g)=\frac{\sin \left(r \cos ^{-1} g\right)}{\sqrt{1-g^{2}}}=\left(\begin{array}{l}
r \\
1
\end{array}\right) g^{r-1}-\left(\begin{array}{l}
r \\
3
\end{array}\right) g^{r-3}\left(1-g^{2}\right)+\left(\begin{array}{l}
r \\
5
\end{array}\right) g^{r-5}\left(1-g^{2}\right)^{2}+\ldots
$$

Thus, using (2.71) we may write $(2.68)$ as

$(2.74) \underline{E}\left(k_{1}+r\right)=\sum_{i=2} c_{i} P_{r}\left[g\left(\sigma_{i}\right)\right] \underline{x}_{i}+h P_{r}\left[g\left(\sigma_{s}\right)\right] \underline{y}+h r\left(\frac{2}{\sigma_{1}(d-b)}\right)_{\frac{u_{r-1}\left[g\left(\sigma_{s}\right)\right]}{w_{r}\left[g\left(\sigma_{1}\right)\right]} \underline{x}_{s}}^{u_{1}}$.

Since $b \leq \sigma_{s} \leq d, g\left(\sigma_{s}\right)$ can lie between - I snd +1 . whis, from Eq. (2.73) we see that $\left|U_{r-1}\left(g\left(\sigma_{s}\right)\right)\right|<r$. Since $1 / \mathbb{T}_{r}\left[g\left(\sigma_{1}\right)\right]$ behayes as $\alpha^{r}$, where $|\alpha|<1$, we have that $\lim E\left(k_{1}+r\right)=0$. Thus, the Chebyshev folynomial method is still a convergent process when principal vectors of grade 2 are present in the set of basis rectors.

The most slowly decaying basis vector in (2.74) will depend on the values of $\sigma_{s}$ and $r$. Except for the vector $\underline{x}_{s}$, ail basis vectors in the expansion (2.74) are modified in the normal Chebyshev way. In spplying the $r$-th degree Chebysher polynomial to $\underline{x}\left(k_{1}\right)$, we see from $E q \cdot(2.74)$ that the $\underline{x}_{s}$ vector has been multiplied by a factor of 


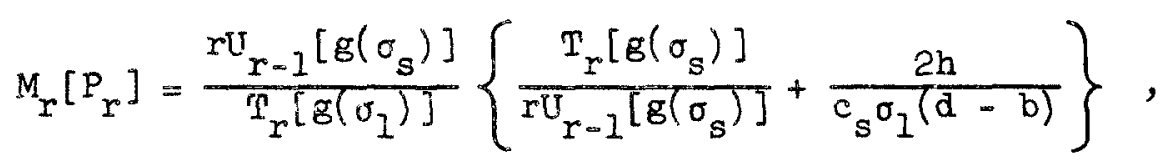

where we assume that $U_{r-1}\left[g\left(\sigma_{s}\right)\right] \neq 0$.

In Table 2.3, we give the values of $M_{r}$ and $M_{r}\left[P_{r}\right]$ when $d=.99$, $\mathrm{b}=0$, and $\mathrm{h} / \mathrm{c}_{\mathrm{s}} \sigma_{1}=1$. In the first case we take $\sigma_{s}=\sigma_{2}$ and in the second case we take $\sigma_{S}=0$. Note that the magnitude of the multiplication factor of the Chebyshev polynomial method did not change much for the two cases ${ }^{1}$ whereas that corresponding to the power method did. Also from Table 2.3, we see that the Chebyshev polynomial iterations would aiverge if a polynomial of degree less than 40 were repeatedly applied.

The presence of principal vectors of grade 2 or higher in the set of basis vectors also makes it very difficult to estimate the parameters needed for the efficient use of the chebysher polynomial method. In general, it is felt that extreme caution should be exercised when using the Chebyshev polynomial method of iteration if the set of eigenvectors for $G$ does not span the associated vector space. We remark that $M_{r}\left[P_{r}\right]$ would be much smaller for the $\sigma_{s}=0$ case if we had taken
$b=-d$. 


\begin{tabular}{|c|c|c|c|c|}
\hline \multirow{2}{*}{$r$} & \multicolumn{2}{|c|}{ CASE $1 ; \sigma_{s}=\sigma_{2}$} & \multicolumn{2}{c|}{ CASE $2 ; \sigma_{s}=0.0$} \\
\cline { 2 - 5 } & $M_{r}$ & $M_{r}\left[{ }_{r}\right]$ & $M_{r}$ & $M_{r}\left[P_{r}\right]$ \\
\hline 1 & 1.990 & 2.960 & 1.000 & 1.000 \\
\hline 2 & 2.960 & 8.396 & .000 & -6.547 \\
\hline 3 & 3.911 & 16.163 & .000 & 14.478 \\
\hline 4 & 4.842 & 24.871 & .000 & -23.378 \\
\hline 5 & 5.754 & 35.878 & & 34.585 \\
\hline 10 & 10.040 & 53.615 & & -53.087 \\
\hline 20 & 17.341 & 29.233 & & -29.161 \\
\hline 40 & 27.698 & 1.277 & & -1.276 \\
\hline 80 & 36.611 & .003 & & -.003 \\
\hline 160 & 32.370 & & & \\
\hline 320 & 12.965 & & & \\
\hline 640 & 1.040 & & & \\
\hline
\end{tabular}

TABLE 2.3

D. Principal Vectors of Grade Greater Than Two

Suppose now that the nonlinear elementary divisor of $G$ is of degree $m+1$, where $m$ is artitrary. For this case we take as a basis for $V_{n}(C)$, the $\mathrm{n}$ vectors

$$
\underline{x}_{1}, \underline{x}_{2}, \ldots, \underline{x}_{s}, \underline{y}_{1}, \underline{y}_{2}, \ldots, \underline{y}_{m}, w_{S+1}, \ldots, \underline{x}_{t},
$$

where the vectors $\underline{x}_{i}$ are eigentectors of $G$ with corresponding eigenvalue $\sigma_{i}$ and the vector $\underline{y}_{j}, j=i, 2, \ldots, m$ is a principal vertor of grade $j+1$ sorresponding 
to the eigenvalue $\sigma_{s}$. The vectors $\underline{y}_{j}$ satisfy the relationship $\underline{y}_{j}=\left(G-\sigma_{s} I\right)^{m-j} \underline{y}_{m}$ or equivalently

$(2.77)$

$$
\left\{\begin{array}{c}
\left(G-\sigma_{s} I\right) y_{m}=y_{m-1} \\
\left(G-\sigma_{s} I\right) y_{n-I}=\underline{y}_{m-2} \\
\vdots \\
\left(G-\sigma_{s} I\right) y_{-2}=y_{-I} \\
\left(G-\sigma_{s} I\right) y_{1}=x_{s}
\end{array}\right.
$$

The existence of such a basis is guaranteed by Theorem 2.1. We also assume that the eigenvalues of $G$ are real and that $\left|\sigma_{1}\right|>\left|\sigma_{2}\right| \geq\left|\sigma_{1}\right|$ for $i \geq 3$. In terms of the besis vectors $(2.75)$, the eigenvector estimate after $\mathrm{k}_{1}$ iterations may be expressed as

$$
\underline{x}\left(k_{1}\right)=\underline{x}_{1}+\sum_{i=2} c_{1} \underline{x}_{i}+\sum_{\substack{j=1 \\ j=1}}^{n} h_{j} \underline{y}_{j}
$$

where we again sssume that the eigenvalue eatimates $\sigma\left(k_{1}+r\right), N \geq 0$, are sufficiently close to $\sigma_{2}$.

For the power method of iteration, the error vector $E\left(k_{1}+r\right)$ may be expressed as

$$
E\left(k_{1}+r\right) \approx \sum_{i=2}\left(\frac{\sigma}{\sigma_{1}}\right)^{r} c_{i} x_{i}+\sum_{j=1}^{m}\left|\frac{G}{\sigma_{1}}\right|^{r} h_{j} \underline{y}_{j}
$$

which after some manipulation may be written as 


$$
\underline{E}\left(k_{1}+r\right) \approx \sum_{i=2}\left(\frac{\sigma_{i}}{\sigma_{1}}\right)^{r} c_{i} \underline{x}_{i}+\left(\frac{\sigma_{s}}{\sigma_{1}}\right)^{r-m}\left[D_{0} \underline{x}_{s}+\sum_{j=1}^{m} D_{j} \underline{y} j\right],
$$

where

$$
D_{j}=\frac{1}{\left(\sigma_{1}\right)^{m}} \sum_{k=0}^{m-j}\left(\begin{array}{l}
r \\
k
\end{array}\right)^{\left(\sigma_{s}\right)^{m-k_{h}}}, \quad j=0,1, \ldots, m,
$$

and where $h_{0}$ is taken to be zero. Since $\sigma_{l}, \sigma_{s}, m$, and the $h_{j}$ 's are independent of $r$ and finite, there exists nonnegative constants $\tilde{D}_{j}$ such that

$$
\left|D_{j}\right| \leq \tilde{D}_{j}\left|\begin{array}{l}
r \\
j
\end{array}\right|, j=0,1, \ldots, m
$$

Thus, since $\lim _{r \rightarrow \infty}(r)^{m}\left(\sigma_{s} / \sigma_{1}\right)^{r-m}=0$, again we have that $\lim _{r \rightarrow \infty} E\left(k_{1}+r\right)=\underline{0}$. From $(2.79)$ and $(2.80)$, we see that the coefficient of the $\underline{x}_{S}$ basis vector in the expansion (2.78) goes to zero as

$$
\left(\begin{array}{l}
r \\
m
\end{array}\right)\left(\frac{\sigma_{s}}{\sigma_{1}}\right)^{r-m}
$$

Thus, for large $\mathrm{m}$, the presence of principal vectors of grade $\mathrm{m}$ may greatly reduce the convergence rate of the power method.

If the eigenvector estimate $\underline{x}\left(k_{1}+r\right)$ had been obtained by applying the r-th degree polynomial $P_{r}[g(G)]$ of $E q .(2.66)$ to $\left.\underline{x}_{\left(k_{1}\right.}\right)$, then the error vector $\underline{E}\left(k_{1}+r\right)$ may be expressed as

$$
\underline{E}\left(k_{1}+r\right) \approx \sum_{i=2} c_{i} P_{r}[g(G)] \underline{x}_{i}+\sum_{j=1}^{m} h_{j} P_{r}[g(G)] \underline{y}_{j}
$$


In a manner similar to that given previously for the case $m=1$, we obtain

$$
\begin{aligned}
\underline{E}\left(k_{1}+r\right) \approx & \sum_{i=2} c_{i} P_{r}\left[g\left(\sigma_{i}\right)\right] \underline{x}_{i}+\sum_{j=1}^{m} h_{j} P_{r}\left[g\left(\sigma_{s}\right)\right] \underline{y}_{j} \\
& +\left\{\sum_{j=1}^{m} \frac{h_{j}}{j !} \frac{d^{j}\left\{P_{r}\left[g\left(\sigma_{s}\right)\right]\right\}}{d z}\right\}_{-s}+\left\{\sum_{j=1}^{m-1} \frac{h_{j+1}}{j !} \frac{d{ }^{j}\left[P_{r}\left[g\left(\sigma_{s}\right)\right]\right\}}{d z^{j}}\right\} \underline{y}_{1} \\
& +\ldots+h_{m} \frac{d\left\{P_{r}\left[g\left(\sigma_{s}\right)\right]\right\}}{d z} \underline{y}_{m=l} .
\end{aligned}
$$

To show that the error vector given by (2.83) approaches the null vector as $r$ approaches infinity, one needs to show that

$$
\lim _{r \rightarrow \infty} \frac{d^{j}\left\{P_{r}\left[g\left(\sigma_{s}\right)\right]\right\}}{d z^{j}}=0
$$

for $j=0,1, \ldots$, m. With $g\left(\sigma_{1}\right) \geq 2.0$ or equivalently with $d \leq \frac{2}{3}+\frac{b}{3}$, one may easily show that $(2.84)$ is true. Wish $d<1.0$, we conjecture that $(2.84)$ is true.

We now turn to the practical problems of estimating the constants $a$ and $b$, and of terminating the iterative process. 
III. THE ESTIMATION OF THE DOMINANCE RATIO A AND MHE ITERMINATION OF THE ITERATTVE PROCESS

In this chapter we shall give a criterion for terminating the iterative process (2.12) and shall specify a numerical means by which to estimate the dominance ratio $d$. For the purposes of this chapter we shall assume that the eigenvalues of the $n \times n$ matrix $G$ are real and are ordered such that

$$
\sigma_{n} \leq \nabla_{n=1} \leq \cdots \leq \sigma_{3} \leq \sigma_{2} \leq \sigma_{1}
$$

and that the set of eigenvectors for $G$ inciules a basio for $v_{n}(C)$. We also assume that $\sigma_{1}>\sigma_{2}>\left|\sigma_{i}\right|$ for $i \geq 3$.

1. The Estimation of d and b

As mentioned previously, the use of non-optimum ilues for $d$ and $b$ can result in a sizable reduction in the convergense rate of the Chebyshev method of iteration. Thus, one is saced with the problem of determining these unknown constants in order to use the Chebyshev polynomial method efficiently。

Since we have assumed that $\mathrm{d}\rangle|\mathrm{b}|$, the rate of convergence of the Chebyshev polynomial method will be governed primarily by the vaiue of d. Thus, the estimate for a appears to be the more important estimate. Hen e, in what follows we assume that enough is known about the eigenvalues of $a$ so that an estimate $b_{C}$ for $b$ may be picked to sarisfy $b_{0}<b$ and $\left|b_{0}\right|<d$. We remark that these conditions on the choice of $b_{0}$ are not impracsical. Sor if the eigenvalues of $G$ are all positive, then $b>0$ and $b_{0}-0$ is a satisfactory choice. If nothing is known about $b$, then one may apply the chebysher polynomial method with the argument matrix $G^{2}$ instead of $G$. Since the eigenwalues 
of $G^{2}$ must be nonnegative, zero is a lower bound for the eigenvalues of $G^{2}$. We remark that the effective convergence rate of the Chebyshev polynomial method with argument matrix $G^{2}$ differs very little from that with argument matrix $G$. In fact, if $b=-d$, then the effective convergence rates of the two are identical. This follows from the identity $T_{2 r}(x)=m_{r}\left(2 x^{2}-1\right)$. The rate of convergence will not be critically affected by the estimate $b_{0}$ if $b_{0}$ satisfies the above two conditions. This then is why we feel justified in assuming that $b_{0}$ satisfy only the two conditions given above and that an accurate estimate for $b$ is not essential. ${ }^{1}$ Thus, henceforth, we will be concerned only with estimates for $d$.

In order to obtain an accurate estimate for $d$, we propose the following strategy. Before starting the Chebyshev method of iteration, do a few (say 5 or 10) power iterations in order to obtain an initial estimate for $a$. (These initial power iterations also provide a reasonable estimate for $\sigma_{1}$ for use in the initial Chebyshev iterations.) Then apply repeatedly low degree Chebysher polynomials so that the estimates for a may be continuously updated. After a good estimate for d is obtained high degree polynomials may be applied, if needed.

Numerical estimates for $d$ may be obtained by observing the decay rate of the residual vector $\underline{y}(k)=\underline{v}(k)-\underline{x}(k-1)$, where $\underline{v}(k)$ and $\underline{x}(k-1)$ are defined by (2.12). We define the residual vector quotient as

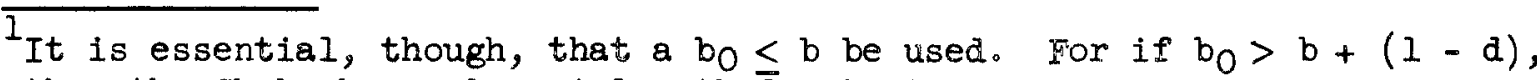
then the Chebyshev polynomial metho $\bar{d}$ will diverge.
} 


$$
Q(k)=\frac{\|\underline{y}(k)\|}{\|\underline{y}(k-1)\|}
$$

where |||| denotes some suitable vector norm. For the power method, it is known that $\lim _{k \rightarrow \infty} Q(k)=d$. To see why this is true, let $\underline{x}(0)$ be expanded in terms of the eigenvectors of $G$ as

$$
\underline{x}(0)=\underline{x}_{1}+\sum_{i=2}^{n} c_{i=1} \underline{x}_{i} .
$$

Thus, we have

$$
\begin{aligned}
& \underline{v}(k)-\underline{x}(k-1)=\frac{\left(\sigma_{1}\right)^{k-1}}{\sigma(0) \cdots \sigma(k-2)}\left\{\left|\frac{\sigma_{1}}{\sigma(k-1)-1}\right| \underline{x}_{1}+\left|\frac{\sigma_{2}}{\sigma(k-1)}-1\right| d^{k-1} c_{2} x_{2}\right. \\
& \left.+\sum_{i=3}^{n}\left(\frac{\sigma_{i}}{\sigma(k-1)}-1\right)\left(\frac{\sigma_{i}}{\sigma_{1}}\right)^{k-1} c_{i-i} \underline{x}_{i}\right\} \text {. }
\end{aligned}
$$

Since $\lim _{k \rightarrow \infty} \sigma(k)=\sigma_{1}$ and $\left|\sigma_{i}\right| / \sigma_{1}<d$ for $i \geq 3$, we have that $\lim _{k \rightarrow \infty} Q(k)=d$. Thus, an initial estimate for $\alpha$ may be obtained by doing a few power iterations before starting the use of Chebyshev polynomials.

Estimates for d may also be obtained every Chebysher iteration by comparing the convergence rate actually being obtained with the theoretical convergence rate one would obtain if the $d$ being used were correct. If a Chebyshev polynomial using $d_{0}$ as the estimate for $d$ is started on iteration $k_{1}+1$ and if 
(3.2) $\left\{\begin{array}{cc}\text { (i) } \quad \sigma\left(k_{1}+r\right) \text { is sufficiently close to } \sigma_{1} \text { for all } r \geq 0 \\ \quad \text { and } \\ \text { (ii) }\left\|\sum_{i=3}^{n} P_{r, d_{0}}\left(\sigma_{i} / \sigma_{1}\right) c_{i} \underline{x}_{1}\right\| \text { is smail relative toll } P_{r, d_{0}}(d) c_{2} \underline{x}_{2} \|,\end{array}\right.$

then from Eq. (2.9) we may approximate $\underline{x}\left(k_{1}+r\right)$ by

$$
\underline{x}\left(k_{1}+r\right) \approx P_{r, \dot{a}_{0}}(I)\left\{\underline{x}_{1}+\frac{P_{r, d}(d)}{P_{r, d_{0}}(I)} c_{2} \underline{x}_{2}\right\},
$$

where $\mathrm{P}_{\mathrm{r}, \mathrm{d}_{0}}(\mathrm{y})$ is given by Eq. (2.17). Since $\mathrm{P}_{\mathrm{r}_{,} \mathrm{d}_{0}}(1)=1$, the residual vector $\underline{\mathrm{y}}\left(\mathrm{k}_{1}+\mathrm{r}+\mathrm{l}\right)$ may be approximated by $\underline{\mathrm{y}}\left(\mathrm{k}_{1}+\mathrm{r}+1\right) \approx(\mathrm{d}-1) \mathrm{P}_{\mathrm{r}, \mathrm{d}_{0}}(\mathrm{~d}) \mathrm{c}_{2} \underline{x}_{2}$ and the residual vector quotient by

$$
Q\left(k_{1}+r+1\right) \approx\left|\frac{P_{r, d}(d)}{P_{r-1, d_{0}}(d)}\right| \text {, }
$$

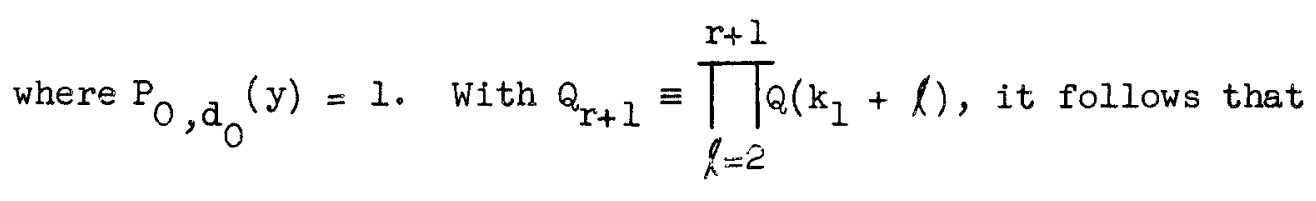

$$
Q_{r+1} \approx\left|P_{r, d_{0}}(d)\right|
$$

Thus, $Q_{r+1}$ gives a measure of $\left|P_{r_{2} d_{0}}(d)\right|$ and one may obtain a new estimate for the dominance ratio by solving (3.4) for $\alpha$. We now shall describe how one may obtain a new estimate for d from (3.4).

Case 1:

$$
I>Q_{r+1}>P_{r, a_{0}}\left(d_{0}\right)
$$

From Figure 3.1, this case implies that $d>d_{0}$ and we are not obtaining the expected convergence rate from the present estimate $d_{0}$. Thus, a new estimate 
for $d$ should be obtained for possible use in the generation of a new Chebyshev polynomial. To obtain this new estimate for d we make use of (3.4). Using $P_{r, d_{0}}(d)$ as defined in $(2.17)$, we may express $(3.4)$ as

$$
Q_{r+1} \approx \frac{T_{r}\left(\frac{2 d-d_{0}-b_{0}}{d_{0}-b_{0}}\right)}{T_{r}\left(\frac{2-d_{0}-b_{0}}{d_{0}-b_{0}}\right)}
$$

or equivalently since $P_{r, d_{0}}\left(a_{0}\right)=1.0 / T_{r}\left(\frac{2-d_{0}-b_{0}}{d_{0}-b_{0}}\right)$

$$
T_{r}\left(\frac{2 d-d_{0}-b_{0}}{d_{0}-b_{0}}\right) \approx \frac{Q_{r+1}}{P_{r, d_{0}}\left(d_{0}\right)}
$$

The right side of (3.6) is greater than one so that the largest positive solution to (3.6) can be expressed as

$$
\mathrm{d} \approx\left(\frac{\mathrm{d}_{0}-\mathrm{b}_{0}}{2}\right)\left\{\cosh \left[\frac{\cosh ^{-1}\left\{\frac{\mathrm{Q}_{r+1}}{\mathrm{P}_{r, d_{0}}\left(\mathrm{a}_{0}\right)}\right\}}{\mathrm{r}}\right]+\left(\frac{\mathrm{d}_{0}+\mathrm{b}_{0}}{\mathrm{~d}_{0}-\mathrm{b}_{0}}\right)\right\}
$$

This solution may then be used as the new estimate for do One may easily show that the d given by (3.7) satisfies the inequality $d_{0}<d<1$.

Case 2:

$$
Q_{r+1}<P_{r_{,} d_{0}}\left(d_{0}\right)
$$

From Figure 3.1 , we see that this case implies that $d<d_{0}$ and we are getting a convergence rate which is greater than that expected from using $\mathrm{d}_{0}$. For this case the right side of $(3.6)$ is less than one and a solution to $(3.6)$ is 


$$
a \approx\left(\frac{a_{0}-b_{0}}{2}\right)\left\{\cos \left[\frac{\cos ^{-1}\left\{\frac{a_{r+1}}{P_{r, d_{0}}\left(d_{0}\right)}\right\}}{r}\right]+\left(\frac{a_{0}+b_{0}}{a_{0}-b_{0}}\right)\right\}
$$

If the principal value is used for the inverse cosine, then the resulting d estimate satisfies $\mu \leq d<d_{0}$, where $\mu$ is the largest positive root of $P_{r, \alpha_{0}}(y)=0 . \quad$ (See Figure 3.1.)

Case 3: $\quad Q_{1+1}>1.0$

This case implies that there has been no error reduction. If the error is not being reduced, then one or more of our assumptions are not being satisfied. This can happen

(a) if $\sigma\left(k_{1}+r\right)$ is not a sufficiently good approximation of $\sigma_{1}$,

(b) if the set of eigenvectors of $G$ does not span $V_{n}(C)$,

(c) If the eigenvalues of $G$ are not all real, or

(d) if $b<b_{0}$.

If the assumptions given in (3.2) are valid, expressions (3.7) and (3.8) normally will give a good estimate for $d$. Obviously, these assumptions do not always hold. However, they may be reasonable under certain conditions. The Chebyshev strategy given below is designed toward this end.

\section{Chebyshev Strategy}

Basically, the Chebyshev strategy can be divided into three parts, as follows: 
5

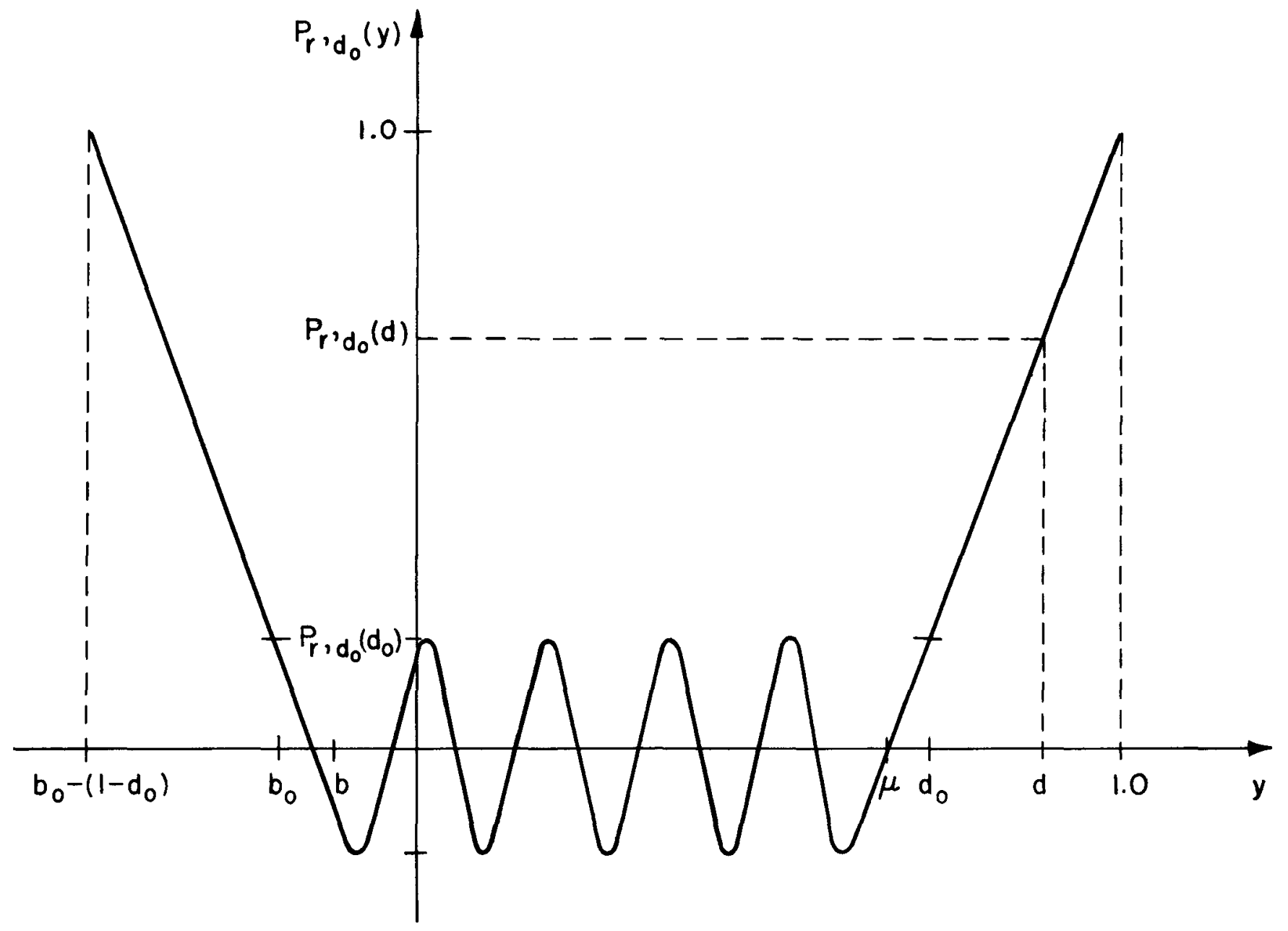

FIG. 3.1 
(a) Initially, at least four iterations of the power type are carried out in order to obtain an initial estimate $d_{0}$ for $a$ and a reasonable estimate for $\sigma_{1}$. We note that these power iterations wlll practically eliminate from the elgenvector guess $\underline{x}(0)$ those eigenvector modes corresponding to the smaller eigenvalues. (see Eq. (2.5))。

(b) The use of thebyshev poiynomials is then started on iteration 5 , say, using $d_{0}$ as the estimate for $d$. Low degree Chebysher polynomials are repeatedly applied with the estimates for the dominance ratio being continuousiy updated. If the low degree Chebyshev polynomials are generated with the dominance ratio under-estimated, these polynomials will greatly reduce all the eigenvector modes in the guess vector $\underline{x}(0)$ except those with the larger eigenvalues. For example, if $d=.889$ and if a 5-th degree Chebyshev polynomial is generated with $d_{0}=.8$, then all eigenvector modes $\underline{x}_{i}$ with $\left(\sigma_{i} / \sigma_{1}\right) \leq .8$ are multiplied by a factor smaller in magnitude than .017 , while the $\underline{x}_{2}$ eigenvector mode is multiplied by a factor of only .2ll. Thus, generating a poiynomial with the dominance ratio under estimated results in assumption (ii) of (3.2) being more nearly satisfied. One nay impose upper bounds on the initial d estimates in an effort to make these estimates less than d. For example, one may insist that $a_{0} \leq .9, x_{1} \leq .925$, etc.

(c) As (3.2) becomes more nearly satisfied giving relatively good convergence towards the correst, $a$, high degree chebyshev polynomials may be applied, if needed, to reduce those eigenvector modes with the larger eigenvalues.

In summary, the Chebyshev strategy is to first eliminate the more rapidly decaying eigenvector modes from the guess $x(0)$ and then concentrate on the most slowly decaying modes. This generally enables the estimates for $a$ to converge to the correct value. 
The decision whether to terminate the present Chebyshev polynomial and start the generation of a new polynomial using an improved estimate for d can be made by comparing the convergence rate actually being obtained with the theoretical convergence rate one would obtain if the estimate for $d$ were correct.

The convergence rate for iteration $\mathrm{k}$ is defined to be

$$
\theta(k) \equiv-\ln \frac{\|\underline{E}(k)\|}{\|\underline{E}(k-1)\|}
$$

where $\underline{E}(k) \equiv \underline{x}(k)-\underline{x}_{1}$ is the error vector for iteration $k$. If a Chebyshev polynomial using $d_{0}$ as the estimate for $d$ is started on iteration $k+l$, then from Eq. (2.9) we may write $\underline{E}(k+r)=\sum_{i=2} c_{i} P_{r}, d_{0}\left(g_{i} / \sigma_{1}\right) \underline{x}_{i}$. Using assumption (3.2) and $\mathrm{Eq} \cdot(3.3), \theta(\mathrm{k}+\mathrm{r})$ may be approximated by

$$
\theta(k+r) \approx-\ln \left|\frac{P_{r, d_{0}}(d)}{P_{r-1, a_{0}}(d)}\right| \approx-\ln [Q(k+r+1)] .
$$

Now if $\mathrm{d}_{0}=\mathrm{d}$, the Chebyshev theory of Chapter II implies that the theoretical convergence rate for iteration $k+r$ should be $-\ln \left[\mathrm{P}_{\mathrm{r}}(d) / \mathrm{P}_{\mathrm{r}-1}(d)\right]$ or equivalently $-\ln \left[T_{r-1}(a) / T_{r}(a)\right]$, where $a=\left(2-a_{0}-b_{0}\right) /\left(a_{0}-b_{0}\right)$. Thus,

$$
(j .10) \quad R(k+r+1)=\frac{\ln [Q(k+r+1)]}{\ln \left[\mathrm{P}_{r-1}(a) / T_{r}(a)\right]}
$$

may be used to compare the actual convergence rate with the theoretical convergence rate for iteration $k+r$.

The decision whether to begin a new Chebyshev polynomial using a new estimate for $d$ can be based on $R(k)$. For example, one could start a new 
polynomial on iteration $k+r+I$ if $R(k+r+1)$ is less than .7 . It is often helpful to insist that all polynomials generated be at least of degree

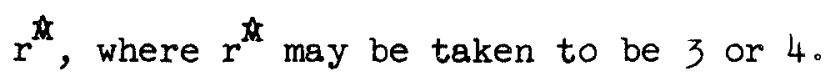

\section{Terminating the Iterative Procedure}

Let the relative sum error $\Lambda(k)$ for iteration $k$ be defined as

$$
\Lambda(\mathrm{k}) \equiv \frac{\|\underline{E}(\mathrm{k})\|_{2}}{\left\|\mathrm{x}_{1}\right\|_{2}}
$$

where by $\|\underline{r}\|_{2}$ is meant the Euclidean or $l_{2}$ norm of the vector $r$, i.e., $\|\underline{r}\|_{\underline{b}} \equiv[\underline{r}, \underline{r}]^{1 / 2}$.

In order to obtain a computable approximation for $\Lambda(k)$ let

$$
\Delta(k) \equiv \frac{\|\underline{v}(k)-\underline{x}(k-1)\|_{2}}{\|\underline{x}(k-1)\|_{2}} .
$$

We now assume $k$ is large enough so that $x(k-1)$ may be approximated by

$$
\underline{x}(\hat{k}-1) \approx \underline{x}_{1}+c_{2} \underline{x}_{2} \text {. }
$$

Thus $\Delta(k)$ may be expressed as

$$
\Delta(k) \approx\left\{\frac{(1-0)^{2} c_{2}^{2} \underline{x}_{2}, \underline{x}_{2}}{\underline{x}_{1}, \underline{x}_{1}+2 c_{2} \underline{x}_{1}, \underline{x}_{2}+c_{2}^{2} \underline{x}_{2}, \underline{x}_{2}}\right\}^{1 / 2} .
$$

Using $(3.13)$, we have $\underline{E}(k-1)=\underline{x}(k-1)-\underline{x}_{1} \approx c_{2} \underline{x}_{2}$ so that $\Lambda(k-1) \approx$ $\left|c_{2}\right| \cdot \| \underline{x}_{2}|| /\left|\underline{x}_{1}\right| \mid$ and 


$$
\Delta(\mathrm{k}) \approx \Lambda(\mathrm{k}-1) \frac{(1-\mathrm{d})}{\left\{1+\mathrm{y}+[\Lambda(\mathrm{k}-1)]^{2}\right\}^{1 / 2}}
$$

where $\mathrm{y}=2 c_{2}\left[\underline{x}_{1}, \underline{x}_{2}\right] /\left[\underline{x}_{1}, \underline{x}_{1}\right]$. Using Schwarz's inequality we have $|y| \leq 2 \Lambda(k-1)$. Thus, if $\Lambda(k-1)<1$, we have

$$
[1-\Lambda(k-1)] \lesssim \frac{(1-d) \Lambda(k-1)}{\Delta(k)} \leqslant[1+\Lambda(k-1)]
$$

Hence, for $\mathrm{k}$ sufficiently large we have

$$
\frac{\Delta(k)}{(1-d)+\Delta(k)} \leqslant \Lambda(k-1) \leqslant \frac{\Delta(k)}{(1-d)-\Delta(k)}
$$

Another possible measure as to how well $\underline{x}(k)$ approximates $\underline{x}_{1}$ is what we shall call the relative point $\operatorname{errcr} \lambda(k)$. If $\underline{e}_{j}$ is a vector of order $n$ whose $j$-th component is unity and all other components zero, then the relative point error for iteration $k$ is defined as

$$
\lambda(k) \equiv \max _{j}\left|\frac{\left[\underline{e}_{j}, \underline{E}(k)\right]}{\left[\underline{e}_{j} \underline{x}_{1}\right]}\right|,
$$

where the subscript $j$ varies only over the set of indices for which $\left(\underline{e}_{j}, \underline{x}_{1}\right) \neq 0$. To obtain a computable approximation for $\lambda(k)$, we let

$$
\delta(k) \equiv \max _{j}\left|1-\frac{\left[\underline{e}_{j}, \underline{v}(k)\right]}{\left[\underline{e}_{j}, \underline{x}(k-1)\right]}\right|,
$$

where the subscript $j$ again varies only over the set of indices for which 


$$
\left(\underline{e}_{j}, \underline{x}(k-1)\right) \neq 0.1
$$

Again assuming that $\mathrm{k}$ is sufficiently large and using Eq. (3.13), we

have

$$
\frac{\left[\underline{e}_{j}, \underline{v}(k)\right]}{\left[\underline{e}_{j}, \underline{x}(k-1)\right]} \approx \frac{\left(\underline{e}_{j}, \underline{x}_{1}\right)+(d) c_{2}\left(\underline{e}_{j}, \underline{x}_{2}\right)}{\left(\underline{e}_{j}, \underline{x}_{1}\right)+c_{2}\left(\underline{e}_{j}, \underline{x}_{2}\right)}
$$

and if $\left(\underline{e}_{j}, \underline{x}_{1}\right) \neq 0$, then

$$
\frac{\left[\underline{e}_{j}, \underline{v}(k)\right]}{\left[\underline{e}_{j}, \underline{x}(k-1)\right]} \approx 1-\frac{(1-d) a_{j}}{1+a_{j}},
$$

where

$$
a_{j}=c_{2} \frac{\left(\underline{e}_{j}, \underline{x}_{2}\right)}{\left(\underline{e}_{j}, \underline{x}_{1}\right)} .
$$

Thus, $\delta(k)$ way be approximated by

$$
\delta(k) \approx(1-d) \max _{j}\left|\frac{a_{j}}{I+a_{j}}\right| .
$$

Using (3.13), $\lambda(k-1)$ may be approximated by $\lambda(k-1) \approx \underset{j}{\max } l_{j} !$. Thus, if $\max _{j}\left|a_{j}\right|<1$, we have

$$
\frac{\lambda(k-1)}{1+\lambda(k-1)} \lesssim \frac{\delta(k)}{1-d} \lesssim \frac{\lambda(k-1)}{1-\lambda(k-1)}
$$

\footnotetext{
In practice, one usually may aroid those $j$ for which $\left(\underline{e}_{j}, \underline{x}_{1}\right)=0$ by allowing the indices $\mathrm{j}$ in $(3.18)$ to vary only orer those $j$ for which $\left[\underline{e}_{j}, \underline{x}(\mathrm{k}-1) \geq\right.$ $\gamma\left\{\max \left[\underline{e}_{j}, \underline{x}(k-1)\right]\right\}$, where $\gamma$ is some Iixed small number.
} 
and

$$
\frac{\delta(k)}{(1-d)+\delta(k)} \lesssim \lambda(k-1) \lesssim \frac{\delta(k)}{(1-d)-\delta(k)}
$$

Thus, one could terminate the 1terative procedure (2.12) by using $\Delta(k)$ and/or $\delta(k)$ modified In some way by a function of d to measure the relative sun and point errors. We note that the relative sum error is an aggregate measure of the error vector $E(k)$ while the relative point error is a polntwise neesure.

$\Delta(k)$ may also be used to estinate the relative eigenvalue error $\tau(\mathbf{k})$, where

$$
\tau(k) \equiv\left|\frac{\sigma(k)}{\sigma_{1}}-1\right|
$$

From Eq. (2.12), $\sigma(k+1) / \sigma_{1}$ is given by

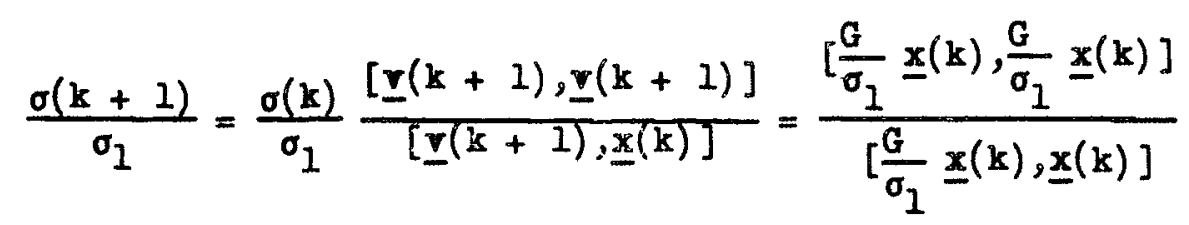

and hence

$$
\frac{\sigma(k+1)}{\sigma_{1}}-1=\frac{\left[\frac{G}{\sigma_{1}} \underline{x}(k),\left(\frac{G}{\sigma_{1}} \underline{x}(k)-\underline{x}(k)\right)\right]}{\left[\frac{G}{\sigma_{1}} \underline{x}(k), \underline{x}(k)\right]} .
$$

If $k$ is large enough so that the rector estimate $\underline{x}(k)$ can be written as $\underline{x}(k) \approx \underline{x}_{1}+c_{2} \underline{x}_{2}$ then $(3.24)$ can be approximeted by

$$
\frac{\sigma(k+1)}{\sigma_{1}}-1 \approx \frac{c_{2}(d-1)\left[\underline{x}_{1}, \underline{x}_{2}+c_{2} d x_{2}, x_{2}\right]}{\underline{x}_{1}, \underline{x}_{1}+c_{2}(1-d) \underline{x}_{1}, \underline{x}_{2}+c_{2}^{2} d x_{2}, \underline{x}_{2}} .
$$


But $[\Lambda(k)]^{2} \approx c_{2}^{2}\left[\underline{x}_{2}, \underline{x}_{2}\right] /\left[\underline{x}_{1}, \underline{x}_{1}\right]$ so that

$$
\left|\frac{\sigma(k+1)}{\sigma_{1}}-1\right| \leqslant(1-d) \Lambda(k)\left[\frac{1+d \Lambda(k)}{(1-d \Lambda(k)(1-\Lambda(k))}\right]
$$

and using (3.15) and the fact that $d<1$, we have

$$
\left|\frac{\sigma(k+1)}{\sigma_{1}}-1\right| \leq\left[\frac{1+\Lambda(k)}{1-\Lambda(k)}\right]^{2} \Delta(k+1) .
$$

The inequalities $(3.16),(3.22)$, and $(3.26)$ are based on the assumption that $k$ is large enough so that

1. the elgenvalue estimates $\sigma(k)$ are sufficiently close to $\sigma_{1}$ and that

2. the efgenvector expansion of the error vector $\underline{E}(\mathrm{k})$ consists of one predominatit eigenvector.

The conditions given above were needed in order to give some mathematical basis for these inequalities. It is felt, however, that the indicated bounds are realistic under much less stringent conditions. In using (3.16) and (3.22), it is important that one have a good estimate for $d$. This is especially true when $a$ is close to unity.

We note that the inequality $(3.26)$ may be sharpened somewhat if the matrix $G$ is symetric. For this case the set of basis vectors may be chosen to be orthogonal. Thus, since $\underline{x}_{1}, x_{-2}=0$, Eqs. $(3.14)$ and $(3.25)$ may be expressed as

$$
\Delta(k+1) \approx\left\{\frac{(1-d)^{2} e_{2}^{2} x_{2}, x_{2}}{x_{1}, x_{1}+c_{2-2}^{2}, x_{-2}}\right\}^{1 / 2}=\frac{(1-d) \Lambda(k)}{\left[1+(\Lambda(k))^{2}\right]^{1 / 2}}
$$


and

$$
\frac{\sigma(k-1)}{\sigma_{1}}-1 \approx \frac{c_{2}^{2} a(d-1) x_{2}, x_{2}}{x_{1}, x_{1}+c_{2}^{2} d x_{2}, x_{2}} \approx \frac{d(d-1)[\Lambda(k)]^{2}}{1+d[\Lambda(k)]^{2}} .
$$

Hence, since $d<1$, we get

$(3.28)$

$$
\left|\frac{\sigma(k+1)}{\sigma_{1}}-1\right| \lesssim \frac{[\Delta(k+1)]^{2}}{1-d}
$$

In the next chapter, we shall discuss some numerical results. 
IV. NUMERTCAL EXAMPLES

In this chapter we give numerical examples which illustrate certain points concerning the behaviox of the Chebyshey polynomial method.

We seek to solve the homogeneous problem

for the dominant eigenvalue $\sigma_{1}$ and its corresponding eigenvector $\underline{x}_{1}$. The Chebyshev iterations are carried ont using the procedure

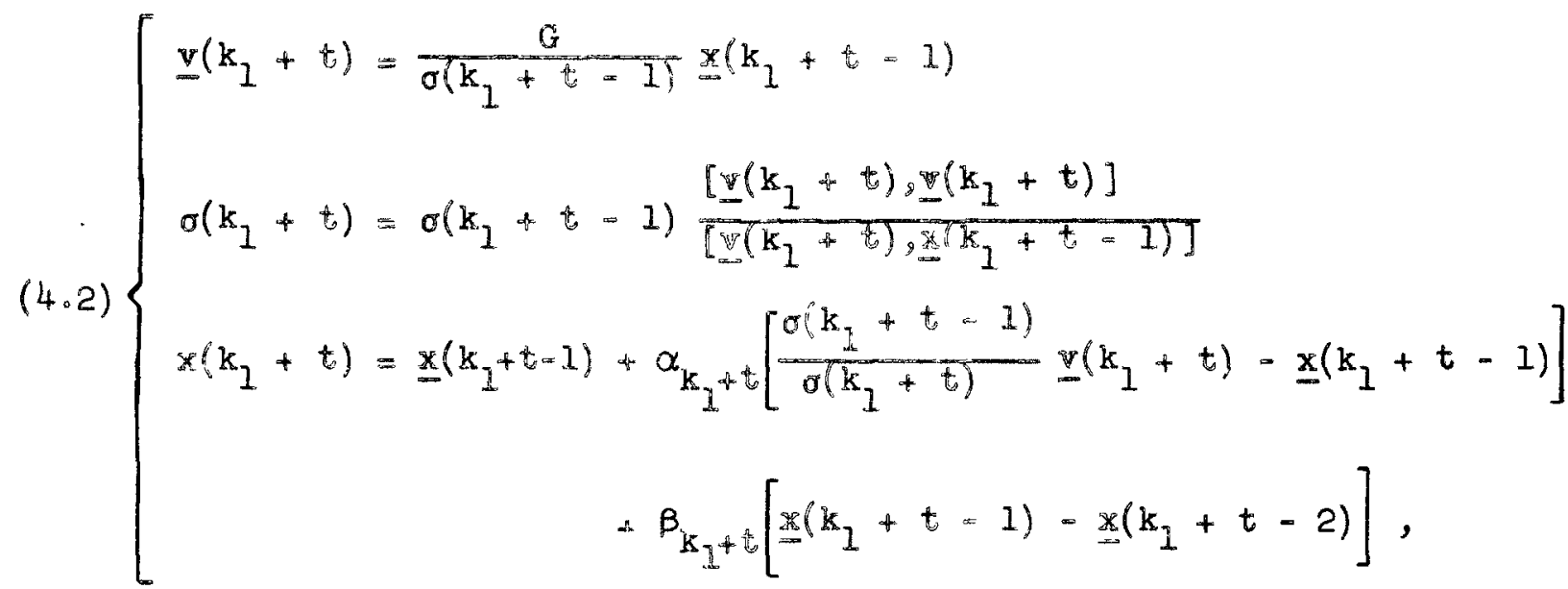

where $\alpha_{k_{1}+t}$ and $\beta_{k_{1}+t}$ are given by $(2.13)$. The above procedure aiffers from

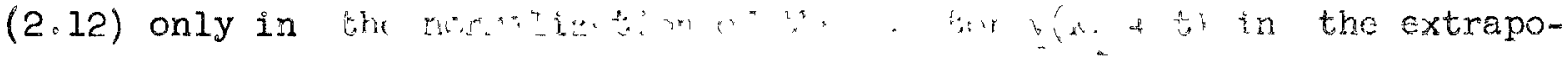
lation. For convergent problems, numerical experiments indicate that both procedures $(2.12)$ and $(4.2)$ give essentially the same results,

The Chebyshev strategy is basicaliy that as described in Chapter III. The generation of a Chebyshew polynomial is terminated and a new polynomial started on iteration $\mathrm{k}$ il 


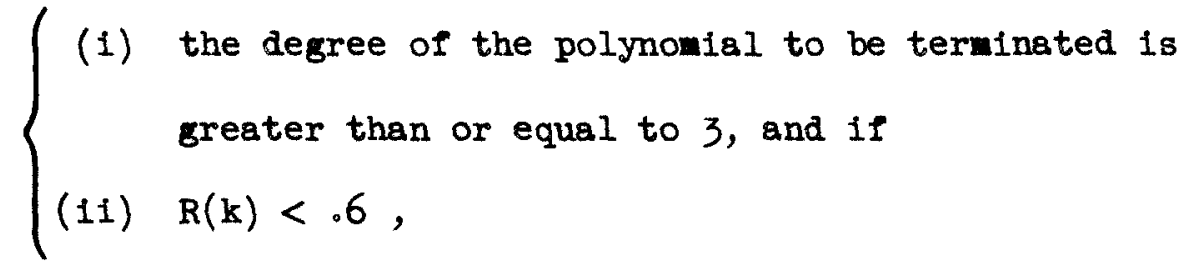

where $R(k)$ is given by $(3.10)$. If it is decided to terminate the $n$-th polynomial then $d_{n+1}$, the estimate for $d$ to be used in the generation of the $(n+1)$ Chebysher polynomial, is usually taken to be the d as determined from (3.7) or (3.8). However, the following restrictions are placed on $d_{n+1}$ : $\mathrm{d}_{0}<.95, \mathrm{~d}_{1} \leq .985, \mathrm{~d}_{2} \leq .995$, and $\mathrm{d}_{\mathrm{n}} \leq .99995$ for $\mathrm{n} \geq 3$.

In the numerical data given below, we let $k=$ the iteration index. $\sigma(k)=$ the estimate for $\sigma_{1}$ alter $k$ iterations。 $d(k)=$ the estimate for $d$ after $k$ iterations.

$r=$ the degree of the Chebysher polynomial which has been generated at the end of the $\mathrm{k}$-th iteration. $\mathrm{r}=0$ implies that the $\mathrm{k}$-th iteration is a power iterate.

$R(k)=$ the ratio of the actual convergence rate to the theoretical convergence rate for iteration $\mathrm{k}-\mathrm{l}, \mathrm{R}(\mathrm{k}) \equiv 1.0$ if iteration $\mathrm{k}-1$ was a power iteration; otherwise $R(k)$ is as defined by $(3.10)$.

$\Delta(\mathrm{k})=$ the error estimate as defined by (3.12)。

$\delta(k)=$ the error estimate as defined by $(3.18)$.

For problem 1 , the matrix $G$ of $(4.1)$ is a symmetric, positive semidefinite matrix of order 99 whose nonzero eigenvalues are $\sigma_{l}=[\cos \pi l / 100]^{2}$, $l=1,2, \ldots, 49$. The remaining 50 eigenvalues of $G$ are equal to zero. Thus, $\sigma_{1}=.999013$ and $d=\sigma_{2} / \sigma_{1}=.99704$. Since the matrix $G$ is symetric for this problem, the set of eigenvectors for $G$ includes an orthonormal basis for the associated vector space. Thus, the real Chebyshev polynomial method of Chapter II may be rigorously applied. 
Three different iteration strategies were used to obtain the dominant eigenvalue for problem 2. First the probiem was run using the Chebyshev strategy described above. When the dominart eigenialue was obtained using only power 1teretions and finslly the problem was solved using only one high degree Chebysher polynomial with the correct value for $d$ being input. The numerical results for probien 1 ere given in Tables $4.1=4.3$. Graphs of $\Delta(\mathrm{k})$ vs. $k$ for the three 1teration strktegies sre given in Figure 4.1 .

\section{Commerts on Problem i:}

From Table 4.1, we see that the chebysher strategy worked as expected for this problem.

The odd behavior of $\mathrm{k}(\mathrm{k}), \Delta(\mathrm{k})$, and $\delta(\mathrm{k})$ in Table 4.3 around the $25 \mathrm{th}$ and 5oth iterations is probably gue to the relationship between the eigenvalues o $l$, of $a$ and the zeros and for peaks of the chebyshev polynomials of degree 24 and 49. Generally, it a bigh degree Chebysher polynomial using an Inpuit vilue for which is too laxge is generated early, the behavior of the $R, \Delta, \delta$ and ousmtities is weh are exratio. This is illustrated in problew 3 below 
PROBLEM 1

CHEBYSAEV ACCELERATI ON

\begin{tabular}{|c|c|c|c|c|c|c|}
\hline $\mathbf{k}$ & $r$ & $R(k)$ & $a(k)$ & $\Delta(\mathbf{k})$ & $\delta(\mathrm{k})$ & $a(k)$ \\
\hline 2 & 0 & 1.00 & .989613 & .04652 & .24965 & .35350 \\
\hline 5 & 1 & 1.00 & .993801 & .01917 & .09994 & .82201 \\
\hline 7 & 3 & .41 & .995651 & .01066 & .03517 & .89142 \\
\hline 8 & 1 & .23 & .996157 & .00872 & .08708 & .90706 \\
\hline 9 & 2 & .55 & .996404 & .00787 & .02740 & .94661 \\
\hline 10 & 3 & .41 & .996810 & .00651 & .02602 & .95272 \\
\hline 11 & 1 & .28 & .997113 & .00553 & .6254 & .95726 \\
\hline 13 & 3 & .60 & .997455 & .00453 & .02367 & .97211 \\
\hline 14 & 1 & .47 & .997679 & .00388 & .01 .392 & .97434 \\
\hline 16 & 3 & .68 & .997869 & .00338 & .01086 & .98144 \\
\hline 17 & 1 & .59 & .998021 & .00297 & .00927 & .98258 \\
\hline 20 & 4 & .69 & .998247 & .00240 & .00625 & .98587 \\
\hline 21 & 1 & .60 & .998349 & .00214 & .00559 & .98758 \\
\hline 25 & 5 & .65 & .998543 & .00166 & .00418 & .99097 \\
\hline 26 & 1 & .58 & .998604 & .00151 & .00362 & .99132 \\
\hline 32 & 7 & .65 & .998784 & .00103 & .00226 & .99345 \\
\hline 33 & 1 & .60 & .998814 & .00093 & .00197 & .99360 \\
\hline 41 & 9 & .64 & .998921 & .00057 & .00126 & .99501 \\
\hline 42 & 1 & .59 & .998932 & .00052 & .00111 & .99511 \\
\hline 50 & 9 & .64 & .998972 & .00036 & .00073 & .99638 \\
\hline 51 & 1 & .60 & .998976 & .00034 & .00065 & .99643 \\
\hline 60 & 10 & .80 & .998997 & .00022 & .00048 & .99696 \\
\hline 61 & 11 & .78 & .998999 & .00021 & .00046 & .99697 \\
\hline 62 & 12 & .76 & .999001 & .00019 & .00045 & .99698 \\
\hline 90 & 40 & .60 & .999013 & .00002 & .00005 & .99704 \\
\hline
\end{tabular}


PROBLEM 1

POWER ITERATIONS

\begin{tabular}{|c|c|c|c|c|c|c|}
\hline $\mathbf{k}$ & $\mathbf{r}$ & $R(k)$ & $\sigma(k)$ & $\Delta(\mathbf{k})$ & $\delta(k)$ & $a(k)$ \\
\hline 2 & 0 & 1.00 & .989613 & .04652 & .24965 & $\cdot 35350$ \\
\hline 5 & 0 & 1.00 & .993801 & .01917 & .09994 & .82201 \\
\hline 7 & 0 & 1.00 & .994808 & .01442 & .07129 & .87426 \\
\hline 8 & 0 & 1.00 & .995155 & .01292 & .06236 & .89593 \\
\hline 9 & 0 & 1.00 & .995439 & .01273 & .05542 & .90854 \\
\hline 10 & 0 & 1.00 & .995678 & .01077 & .04987 & .91841 \\
\hline 11 & 0 & 1.00 & .995883 & .00998 & .04532 & .92634 \\
\hline 13 & 0 & 1.00 & .996215 & .00873 & 03833 & .93831 \\
\hline 14 & 0 & 1.00 & .996353 & .00823 & .03558 & .94294 \\
\hline 16 & 0 & 1.00 & .996588 & .00741 & .03112 & .95036 \\
\hline 17 & 0 & 1.00 & . 996689 & .00706 & .02928 & .95339 \\
\hline 20 & 0 & 1.00 & .996945 & .00621 & .02486 & .96057 \\
\hline 21 & 0 & 1.00 & .997017 & .00598 & .02367 & .96249 \\
\hline-.25 & 0 & 1.00 & .997260 & .00522 & .01986 & .96858 \\
\hline 26 & 0 & 1.00 & .997312 & .00506 & .01909 & .96980 \\
\hline 32 & 0 & 1.00 & .997567 & .00430 & .01547 & .97546 \\
\hline 33 & 0 & 1.00 & .997602 & .00420 & .01500 & .97620 \\
\hline 41 & 0 & 1.00 & .997836 & .00354 & .01203 & .98077 \\
\hline 42 & 0 & 1.00 & .997860 & .00347 & .01174 & .98121 \\
\hline 50 & 0 & 1.00 & .998026 & .00303 & .00983 & .98411 \\
\hline 51 & 0 & 1.00 & .998040 & .00298 & .00963 & .98441 \\
\hline 60 & 0 & 1.00 & .998182 & .00262 & .00815 & .98661 \\
\hline 61 & 0 & 1.00 & .998195 & .00258 & .00802 & .98681 \\
\hline 90 & 0 & 1.00 & .998473 & .00187 & .00535 & .99061 \\
\hline 300 & 0 & 1.00 & .998935 & .00053 & .00129 & .99566 \\
\hline
\end{tabular}


PROBEEM 1

CHEBYSHEV (HIGH DEGREE) ACCELERATION - TNFUT $d=.99704$

\begin{tabular}{|c|c|c|c|c|c|c|}
\hline $\mathbf{k}$ & $r$ & $R(k)$ & $\boldsymbol{\sigma}(\mathbf{k})$ & $\Delta(\mathrm{k})$ & $\delta(k)$ & $\mathbb{d}(\mathrm{k})$ \\
\hline 2 & 1 & 1.00 & .989613 & .04652 & .24965 & .99704 \\
\hline 5 & 4 & 1.08 & .995101 & .02976 & 1.19120 & .98015 \\
\hline $7-$ & 6 & .98 & .996224 & .02488 & 05943 & .99096 \\
\hline 8 & 7 & .99 & .996627 & .02231 & .16 .182 & .992 .83 \\
\hline 9 & 8 & .99 & .997082 & .02985 & .12047 & .99395 \\
\hline 10 & 9 & .99 & .997464 & .01759 & .08013 & . 99468 \\
\hline 11 & 10 & .99 & .997776 & .015551 & $.06: 05$ & $.9951 \%$ \\
\hline 23 & 22 & 1.02 & .998908 & .00371 & .ค1066 & .99570 \\
\hline 24 & 23 & 1.03 & .998921 & .00330 & .00959 & .99672 \\
\hline 25 & 24 & 1.04 & .998929 & .00294 & .00861 & .99675 \\
\hline 26 & 25 & $=.42$ & .998940 & .00305 & .00931 & .99688 \\
\hline 27 & 26 & 4.30 & .998962 & .00192 & .00653 & .99667 \\
\hline 28 & 27 & -1.57 & . 998968 & .00226 & .00674 & .99685 \\
\hline 29 & 28 & 1.50 & .998973 & .00192 & .00617 & .99684 \\
\hline 30 & 29 & .99 & .998979 & .00172 & .00567 & .99685 \\
\hline 50 & 49 & .58 & .999012 & .00022 & .01807 & .99700 \\
\hline 51 & 50 & 8,44 & .999013 & .00009 & 00746 & .99695 \\
\hline 52 & 51 & -7.86 & 999013 & .00020 & .02578 & .99703 \\
\hline 53 & 52 & 3.99 & .999013 & .00013 & .00577 & 99697 \\
\hline 54 & 53 & .19 & .999013 & .00013 & .00816 & .99700 \\
\hline 55 & 54 & .99 & .999013 & .00012 & .00239 & .99700 \\
\hline 56 & 55 & .98 & .999013 & .00010 & .00141 & .99700 \\
\hline 57 & 56 & .98 & .999013 & .00009 & .00105 & .99700 \\
\hline 58 & 57 & .99 & .999013 & .00008 & .00081 & .99700 \\
\hline 71 & 70 & .96 & .999013 & .00002 & .03008 & 99701 \\
\hline
\end{tabular}




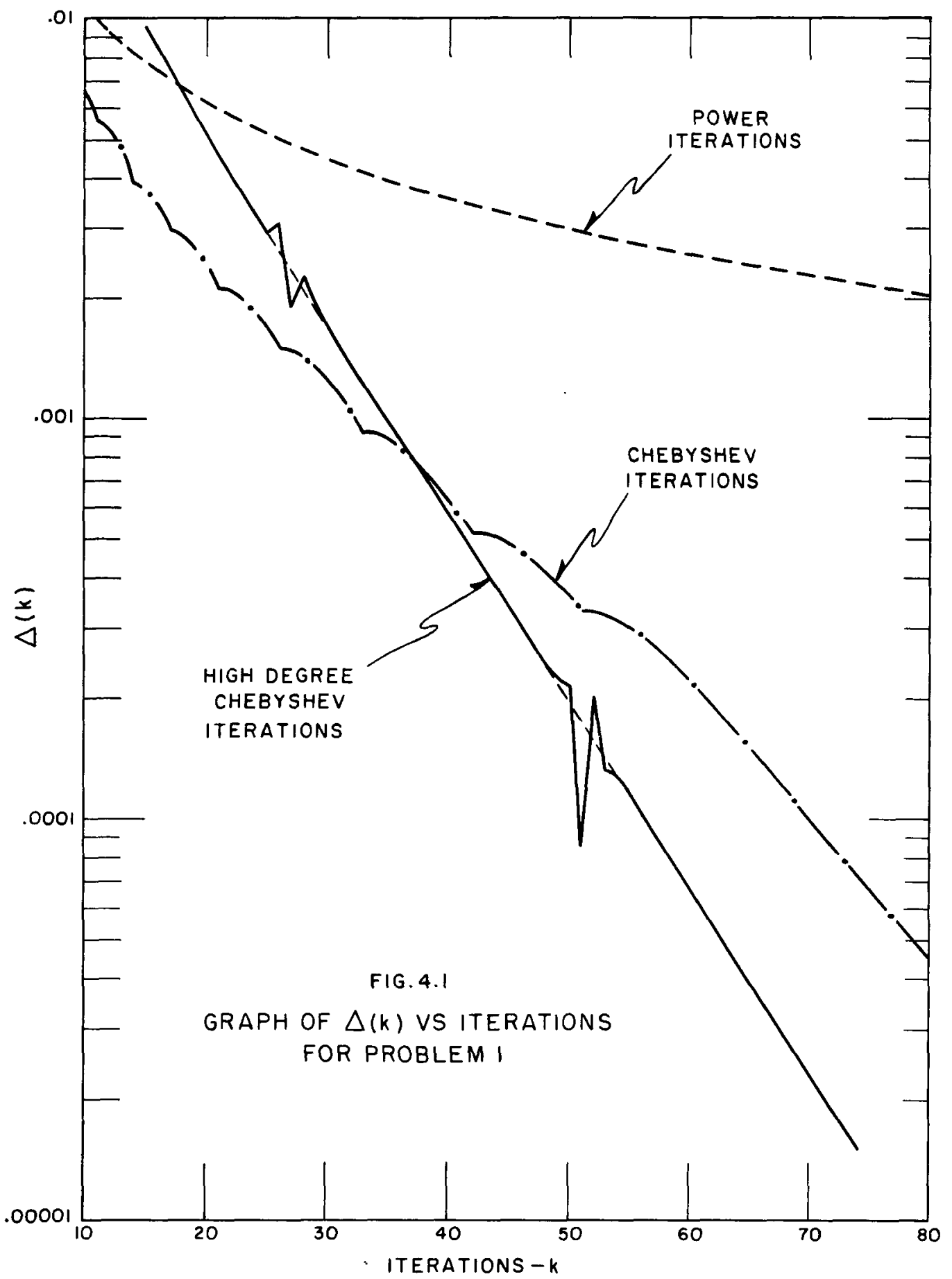


For problem 2, the matrix $G$ has the same eigenvalues as those for problem 1 but the set of eigenvectors for this problem does not include a basis for the associated rector space. A nonlinear elementary divisor of degree 50 is associated with the elgenvalue zero and thus, principal vectors of grade 50 are present in the set of basis vectors for $G$. The numerical results obtained from this problem are given in Tables 4.4-4.7. In Tables 4.6 and 4.7 , the quantity $Q(k)$ is defined by Eq. (3.1).

Comments on Problem 2:

The power iterations for problem 2 were almost identical to those of problem 1. This would not have been the case, however, had the nonlinear divisor been associated with some rather large nonzero eigenvalue.

Note the rapid divergence property of the Chebyshev iterations. The results of the Chebyshev high degree acceleration problems do not necessarily Imply that the conjecture of (2.84) is false. In (2.84) it is assumed that an infinite degree Chebyshev polynomial is generated and that the true dominant eigenvalue is used as the normalizing factor in the Chebyshev extrapolation. In an attempt to see the effect of the normalizing factor, the high degree Chebysher acceleration problem of Table 4.6 was rerun using the true dominant eigenvalue in the Chebyshev extrapolation, 1.e., the $\underline{x}\left(k_{1}+t\right)$ term in (4.2) was computed using $\left[\sigma\left(k_{1}+t-1\right) / \sigma_{1}\right] \underline{v}\left(k_{1}+t\right)$ instead of $\left[\sigma\left(k_{1}+t-1\right) / \sigma\left(k_{1}+t\right)\right] \underline{v}\left(k_{1}+t\right)$. As seen from Tables 4.6 and 4.7, this change seemed to affect only the eigenvalue estimates. The reason for the relative stability of the $R(k), d(k)$, and $Q(k)$ quantities in Tables 4.6 and 4.7 is not known.

The numerical results of problem 2 show that the presence of nonlinear elementary divisors can drastically affect the behavior of the Chebyshev 
polynomial method. We remark, however, that the Chebyshev polynomial method often may be used to good advantage when the nonlinear divisors are associated with very small eigenvalues and when the strategy used makes provisions for the presence of principal vectors in the set of basis vectors. For a dis. cussion of this, see Hageman and Kellogg (1966). 
PROBLEM 2

CHEBYSHEV ACCELERATION

\begin{tabular}{|c|c|c|c|c|c|c|}
\hline $\mathbf{k}$ & $r$ & $\mathrm{R}(\mathrm{k})$ & $o(k)$ & $\Delta(k)$ & $\delta(\mathrm{k})$ & $d(k)$ \\
\hline 2 & 0 & 1.00 & .988735 & .04155 & .24904 & .38662 \\
\hline 5 & 0 & 1.00 & .993636 & - Oamar & .09973 & .82724 \\
\hline 7 & 3 & .43 & .995671 & .00952 & .03573 & .89279 \\
\hline 8 & 1 & .22 & .996184 & .00785 & .02622 & .90927 \\
\hline 9 & 2 & .49 & .996411 & .00718 & .6854 & .95319 \\
\hline 10 & 3 & .34 & .996807 & .00615 & 0957 & .96015 \\
\hline II & 1 & .20 & .997118 & .00548 & .03706 & .96575 \\
\hline 13 & 3 & -1.42 & .997475 & .00715 & .08527 & \\
\hline 14 & 1 & -2.22 & .997743 & .01312 & .19625 & .96575 \\
\hline 16 & 3 & -4.23 & .998267 & .04166 & .57306 & \\
\hline 17 & 1 & -2.94 & .999980 & .09300 & 4.15835 & .96575 \\
\hline 18 & 2 & -5.94 & 1.002620 & .14019 & 6.34790 & \\
\hline 19 & 3 & $-4 \cdot 32$ & 1.022880 & .32197 & 7.57360 & \\
\hline 20 & 1 & -2.92 & 1.161700 & .77061 & 8.96510 & .96575 \\
\hline 22 & 3 & $-2 \cdot 38$ & -7.201190 & 1.97715 & .89157 & \\
\hline 23 & 1 & -.19 & 4.877060 & 1.68904 & 1.38180 & .96575 \\
\hline 26 & 4 & 1.23 & 1.192005 & .83736 & 4.28377 & \\
\hline 27 & 1 & -2.03 & 15.030100 & 1.94680 & 1.14667 & .96575 \\
\hline 51 & 3 & -1.25 & 7.245400 & 2.28150 & 1.09660 & \\
\hline
\end{tabular}

TABLE 4.4 
PROBLEM 2

POWER ITERATIONS

\begin{tabular}{|c|c|c|c|c|c|c|}
\hline$k$ & $r$ & $R(k)$ & $\sigma(k)$ & $\Delta(k)$ & $\delta(k)$ & $d(k)$ \\
\hline 2 & 0 & 1.00 & .988735 & .04155 & .24904 & .38662 \\
\hline 5 & 0 & 1.00 & .993636 & .01712 & .09973 & .82724 \\
\hline 7 & 0 & 1.00 & .994710 & .01301 & .07123 & .88353 \\
\hline 8 & 0 & 1.00 & .995074 & .01170 & .06232 & .89970 \\
\hline 9 & 0 & 1.00 & .995371 & .01067 & .05539 & .91187 \\
\hline 10 & 0 & 1.00 & .995620 & .00983 & .04984 & .92137 \\
\hline 11 & 0 & 1.00 & .995831 & .00913 & .04530 & .92900 \\
\hline 13 & 0 & 1.00 & .996174 & .00803 & .03832 & .94048 \\
\hline 14 & 0 & 1.00 & .996315 & .007584 & .03557 & .94492 \\
\hline 50 & 0 & 1.00 & .998015 & .00287 & .00983 & .98443 \\
\hline 51 & 0 & 1.00 & .998033 & .00282 & .00963 & .98471 \\
\hline 90 & 0 & 1.00 & .998465 & .00179 & .00535 & .99068 \\
\hline 300 & 0 & 1.00 & .998929 & .00049 & .00128 & .99543 \\
\hline
\end{tabular}

TABLE 4.5 
PROBLEM 2

CHEBYSHEV (HIGH DEGREE) ACCELERATION INPIJ $d=.99704$

\begin{tabular}{|c|c|c|c|c|c|c|}
\hline $\mathbf{k}$ & $r$ & $R(k)$ & $\sigma(k)$ & $Q(k)$ & $\delta(k)$ & $a(k)$ \\
\hline 3 & 1 & 1.00 & .991 & .6488 & .1662 & .99704 \\
\hline 5 & 3 &. .08 & .996 & 1.0015 & .4545 & .94707 \\
\hline 7 & 5 & 19.10 & .998 & 2.1296 & 1.5511 & \\
\hline 8 & 6 & 18.45 & 1.0001 & 2.4899 & 1.1932 & \\
\hline 9 & 7 & 16.33 & 1.018 & 2.5046 & 1.9539 & \\
\hline 10 & 8 & 14.43 & 1.180 & 2.6049 & 1.3407 & \\
\hline 11 & 9 & 11.93 & -1.098 & 2.3974 & 1.991 .3 & \\
\hline 25 & 23 &.- .12 & 8.149 & 1.0125 & 1.0044 & \\
\hline 26 & 24 & .006 & 37.401 & .9994 & 1.0155 & \\
\hline 27 & 25 & .09 & 87.462 & .9896 & 1.2289 & \\
\hline 51 & 49 & .73 & 54.195 & .9240 & 1.0416 & \\
\hline 52 & 50 & .73 & 43.764 & .9232 & 1.0005 & \\
\hline 53 & 51 & .74 & 55.134 & .9225 & 1.0007 & \\
\hline 100 & 98 & .89 & 32.889 & 9079 & 1,0091 & \\
\hline 101 & 99 & .89 & 92.769 & .9077 & 1.0014 & \\
\hline 102 & 100 & .89 & 47.791 & .9076 & 1.0033 & \\
\hline 200 & 198 & .95 & 3.920 & .9018 & 1.0001 & .9982 \\
\hline 201 & 199 & .95 & 21.413 & .9018 & 1.0030 & .9982 \\
\hline 202 & 200 & .95 & 79.812 & .9017 & 1.0007 & .9982 \\
\hline 298 & 296 & .97 & 20.268 & .9001 & 1.0022 & .9976 \\
\hline 299 & 297 & .97 & 7.289 & .9000 & 1.0006 & .9976 \\
\hline 300 & 298 & .97 & 67.839 & .9000 & 1.0002 & .9976 \\
\hline
\end{tabular}

TABIE 4.6 
PROBLEM 2

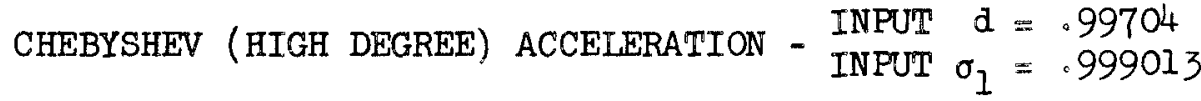

\begin{tabular}{|c|c|c|c|c|c|c|}
\hline $\mathrm{k}$ & $r$ & $R(k)$ & $\sigma(k)$ & $Q(k)$ & $\delta(k)$ & $a(k)$ \\
\hline 3 & 1 & 1.00 & .991 & .6421 & .1662 & .99704 \\
\hline 5 & 3 & -.93 & .996 & 1.0165 & .5151 & .94677 \\
\hline 7 & 5 & 19.06 & .998 & 2.1268 & 1.4248 & \\
\hline 8 & 6 & 18.44 & 1.000 & 2.4895 & 1.1609 & \\
\hline 9 & 7 & 16.33 & 1.021 & 2.5941 & 1.7265 & \\
\hline 10 & 8 & 14.41 & 1.219 & 2.6002 & 1.3235 & \\
\hline 11 & 9 & 11.68 & -.789 & 2.3534 & 3.8515 & \\
\hline 25 & 23 & .10 & 57.687 & .9898 & 1.0360 & \\
\hline 26 & 24 & .18 & 43.993 & .9810 & 1.0015 & \\
\hline 27 & 25 & .24 & 4.244 & .9743 & 1.0265 & \\
\hline 51 & 49 & .74 & 42.202 & .9226 & 1.0015 & \\
\hline 52 & 50 & .75 & 53.643 & .9219 & 1.0177 & \\
\hline 53 & 51 & .75 & 23.402 & .9212 & 1.0418 & \\
\hline 100 & 98 & .89 & 52.409 & .9077 & 1.0049 & \\
\hline 101 & 99 & .89 & 11.303 & .9076 & 1.0027 & \\
\hline 102 & 100 & .89 & 60.198 & .9074 & 1.0027 & \\
\hline 200 & 198 & .95 & $97 \cdot 918$ & .9018 & 1.0017 & .99805 \\
\hline 201 & 199 & .95 & 85.801 & .9018 & 1.0123 & .99804 \\
\hline 202 & 200 & .95 & 76.197 & .9017 & 1.0023 & .99803 \\
\hline 298 & 296 & .97 & 33.731 & .9001 & 1.0027 & .99752 \\
\hline 299 & 297 & .97 & 50.082 & .9000 & 1.0002 & .99752 \\
\hline 300 & 298 & .97 & 28.078 & .9000 & 1.0008 & .99752 \\
\hline
\end{tabular}

TABLE 4.7 
For problem 3, the matrix $G$ is not symmetric but the set of elgenvectors for $G$ is known to include a basis for the associated vector space. It is also known that the eigenvalues of $G$ are nonnegative. The dominant eigenvalue, $\sigma_{1}$, for this problem appears to be .999886 . The numerical results are given in Tables $4.8-4.10$ and graphs of $\Delta(k)$ vs. $k$ for the three iteration strategies are given in Figure 4.2 .

Comments on Problem 3:

Using Table 4.9 , the inequality $(3.22)$ for the relative point error after 300 power iterations gives

$$
.365 \lesssim \lambda_{(299)} \lesssim 1.26
$$

One component of the eigenvector estimate after 300 power iterations had a relative point error of .55 . Thus, the inequality $(3.22)$ can give realistic bounds for $\lambda(k)$. In using $(3.22)$, it is well to keep in mind that a sufficiently good estimate for $d$ is needed. For example, using $d(k)$ as the estimate for $d$, inequality $(3.22)$ gives for iteration 73

$$
.055<\frac{.00118}{.0201+.00118} \leq \lambda(72)<\frac{.00118}{.0201-.00118}<.063
$$

which obviously is not correct.

In Table 4.8, note that a Chebyshev polynomial of degree 12 with $d_{0}=.97689$ was used early and after that low degree polynomials were again used. This implies that the initial guess vector had a rather large error component associated with an eigenvalue $\sigma_{i}$, where $\sigma_{i} / \sigma_{1} \approx .98$. This fact is also implied from the power iterations since from Table 4.9 we see that the 
estimate for a from iteration 30 to iteration 73 varied between only .978 and .980 . One of the nice properties of the Chebyshev polynomial method is that the method, if properly used, can pick out large components in the error vector and reduce them efficiently.

The high degree Chebyshev problem with input $d=.99976$ is converging at a slower rate than the Chebyshev problem with the strategy. This is due to the fact that the error components associated with smaller eigenvalues are being reduced in the high degree problem at a rate dictated by the second largest efgenvalue $\sigma_{2}$. The erratic behavior of $\Delta(k)$ for the high degree Chebyshev problem can be easily seen in Figure 4.2 . 
FROBLEM 3

CHEBYSHEV ACCELERATION

\begin{tabular}{|c|c|c|c|c|c|c|}
\hline $\mathbf{k}$ & $r$ & $\mathrm{R}(\mathrm{k})$ & $\sigma(k)$ & $\Delta(\mathrm{k})$ & $\delta(\mathrm{k})$ & $\mathrm{d}(\mathrm{k})$ \\
\hline 5 & 1 & 1.00 & .997093 & .002691 & .00493 & .90000 \\
\hline 7 & 3 & .32 & .997686 & .002080 & .00366 & .95686 \\
\hline 8 & 1 & .22 & .997973 & .001820 & .00345 & .95000 \\
\hline 10 & 3 & .40 & .998333 & .001539 & .00313 & .97550 \\
\hline 11 & 1 & .35 & .998594 & .001351 & .00283 & .97689 \\
\hline 22 & 12 & .65 & .999693 & .000168 & .00032 & .97979 \\
\hline 23 & 1 & .53 & .999708 & .000142 & .00025 & .98006 \\
\hline 25 & 3 & .38 & .999718 & .000133 & .00025 & .99110 \\
\hline 26 & 1 & .33 & •999727 & .000126 & .00024 & .99194 \\
\hline 29 & 4 & .65 & .999740 & .000114 & .00022 & .99425 \\
\hline 30 & 1 & .55 & .999748 & .000108 & .00021 & .99470 \\
\hline 32 & 3 & .48 & .999751 & .000105 & .00021 & .99707 \\
\hline 35 & 3 & .59 & .999755 & .000102 & .00021 & .99836 \\
\hline 38 & 3 & .61 & .999759 & .000100 & .00020 & .99904 \\
\hline 39 & 1 & .58 & .999760 & .000099 & .00020 & .99908 \\
\hline 42 & 4 & .61 & .999762 & .000098 & .00020 & .99941 \\
\hline 43 & 1 & .55 & .999763 & .000097 & .00020 & .99945 \\
\hline 45 & 3 & .49 & .999764 & .000097 & .00020 & .99971 \\
\hline 46 & 1 & .46 & .999764 & .000097 & .00019 & .99973 \\
\hline 52 & 7 & .60 & .999768 & .000095 & .00019 & .99982 \\
\hline 53 & 1 & .56 & .999769 & .000095 & .00019 & .99983 \\
\hline 63 & 8 & 1.61 & .999772 & .000094 & .00018 & .99991 \\
\hline 73 & 18 & 2.54 & .999788 & .000088 & .00015 & .99988 \\
\hline 83 & 28 & 3.37 & .999813 & .000076 & .00011 & .99985 \\
\hline 93 & 38 & 5.43 & .999842 & .000055 & .00007 & .99982 \\
\hline 103 & 48 & 9.76 & .999867 & .000028 & .00003 & .99979 \\
\hline 110 & 55 & 26.46 & .999881 & .000009 & .00001 & .99976 \\
\hline
\end{tabular}


PROBLEM 3

POWER ITERATIONS

\begin{tabular}{|c|c|c|c|c|c|c|}
\hline $\mathrm{k}$ & $r$ & $R(k)$ & $\sigma(k)$ & $\Delta(\mathbf{k})$ & $\delta(k)$ & $a(k)$ \\
\hline 2 & 0 & 1.00 & .996539 & .00406 & .00899 & .44934 \\
\hline 5 & 0 & 1.00 & .997093 & .00269 & .00493 & .92902 \\
\hline 7 & 0 & 1.00 & .997323 & .00242 & .00377 & .95161 \\
\hline 8 & 0 & 1.00 & .997421 & .00231 & .00374 & .95665 \\
\hline 23 & 0 & 1.00 & .998383 & .00146 & .00303 & .97595 \\
\hline 30 & 0 & 1.00 & .998653 & .00125 & .00269 & .97820 \\
\hline 43 & 0 & 1.00 & .999001 & .00095 & .00213 & .97946 \\
\hline 44 & 0 & 1.00 & .999022 & .00093 & .00209 & .97949 \\
\hline 45 & 0 & 1.00 & .999042 & .00091 & .00205 & .97951 \\
\hline 52 & 0 & 1.00 & .999166 & .00079 & .00179 & .97959 \\
\hline 53 & 0 & 1.00 & .999182 & .00077 & .00176 & .97959 \\
\hline 63 & 0 & 1.00 & .999316 & .00063 & .00144 & .97967 \\
\hline 73 & 0 & 1.00 & .999416 & .00051 & .00118 & .97990 \\
\hline 83 & 0 & 1.00 & .999492 & .00042 & .00096 & .98036 \\
\hline 93 & 0 & 1.00 & .999551 & .00034 & .00078 & .98108 \\
\hline 103 & 0 & 1.00 & .999597 & .00029 & .00064 & .98209 \\
\hline 110 & 0 & 1.00 & .999623 & .00025 & .00056 & .98298 \\
\hline 150 & 0 & 1.00 & .999709 & .00015 & .00026 & .99003 \\
\hline 200 & 0 & 1.00 & .999748 & .00011 & .00021 & .99691 \\
\hline 250 & 0 & 1.00 & .999760 & .00010 & .00020 & .99910 \\
\hline 300 & 0 & 1.00 & .999766 & .00010 & .00019 & .99967 \\
\hline
\end{tabular}

TABLE 4.9 
PROBLEM 3

CHEBYSHEV (ETGH DEUREE) ACCELERANDTON = INFUT \& $=.99976$

\begin{tabular}{|c|c|c|c|c|c|c|}
\hline $\mathrm{k}$ & $r$ & $\mathrm{R}(\mathrm{k})$ & $\sigma(\mathrm{k})$ & $\Delta(k)$ & $\delta(k)$ & $d(k)$ \\
\hline 2 & 1 & 1.00 & .996539 & .004064 & .00899 & .99976 \\
\hline 5 & 4 & $17 \cdot 82$ & .998659 & .002883 & .00736 & $.983: 8$ \\
\hline 7 & 6 & -1.13 & 1.000413 & .002736 & .00620 & .99315 \\
\hline 8 & 7 & 64.65 & 1.001129 & .003823 & .01215 & .99932 \\
\hline 10 & 9 & 7.06 & 1.000933 & .002878 & .00718 & .99767 \\
\hline 11 & 10 & 25.62 & 1.001089 & .003512 & .01233 & .99926 \\
\hline 12 & 11 & 11.67 & 1.000705 & .003150 & .00963 & .99893 \\
\hline 13 & 12 & -4.55 & . 999991 & .003277 & .00942 & .99927 \\
\hline 14 & 13 & 20.41 & .999961 & .004045 & .01264 & \\
\hline 15 & 14 & 36.02 & . 999939 & .002604 & .00532 & . 99891 \\
\hline 42 & 41 & -6.49 & .999502 & .002475 & 00353 & 99971 \\
\hline 43 & 42 & -5.55 & .999638 & .001698 & .00461 & $.9997^{7}$ \\
\hline 44 & 43 & -6.84 & . 999799 & - OOEOE? & .00611 & .99983 \\
\hline 45 & 44 & 9.16 & .999772 & .001578 & .00484 & .99976 \\
\hline 46 & 45 & 11.45 & .999651 & .001153 & .00287 & .99970 \\
\hline 53 & 52 & .02 & 1.000180 & .001130 & .00443 & . 99975 \\
\hline 63 & 62 & 3.49 & .999403 & .000697 & $.0016 ?$ & .99973 \\
\hline 73 & 72 & 10.13 & . 999860 & .000564 & .00147 & .99975 \\
\hline 83 & 82 & -2.53 & .999802 & .000485 & .00135 & $.999^{\prime 76}$ \\
\hline 93 & 92 & -2.41 & 1.000034 & .000299 & .00072 & .99975 \\
\hline 103 & 102 & 16.17 & .999890 & .000273 & .00091 & .99976 \\
\hline 107 & 106 & .92 & .999862 & .000198 & 20044 & .99976 \\
\hline 108 & 107 & 22.47 & .999830 & .000099 & .00031 & .99973 \\
\hline 109 & 108 & -8.46 & .999808 & $.0,00128$ & .00032 & $9997^{1}$ \\
\hline 110 & 109 & $=7.93$ & .999819 & .000164 & $.0 n 038$ & .99975 \\
\hline 111 & 110 & 1.98 & .999842 & .000154 & .20034 & .90073 \\
\hline
\end{tabular}




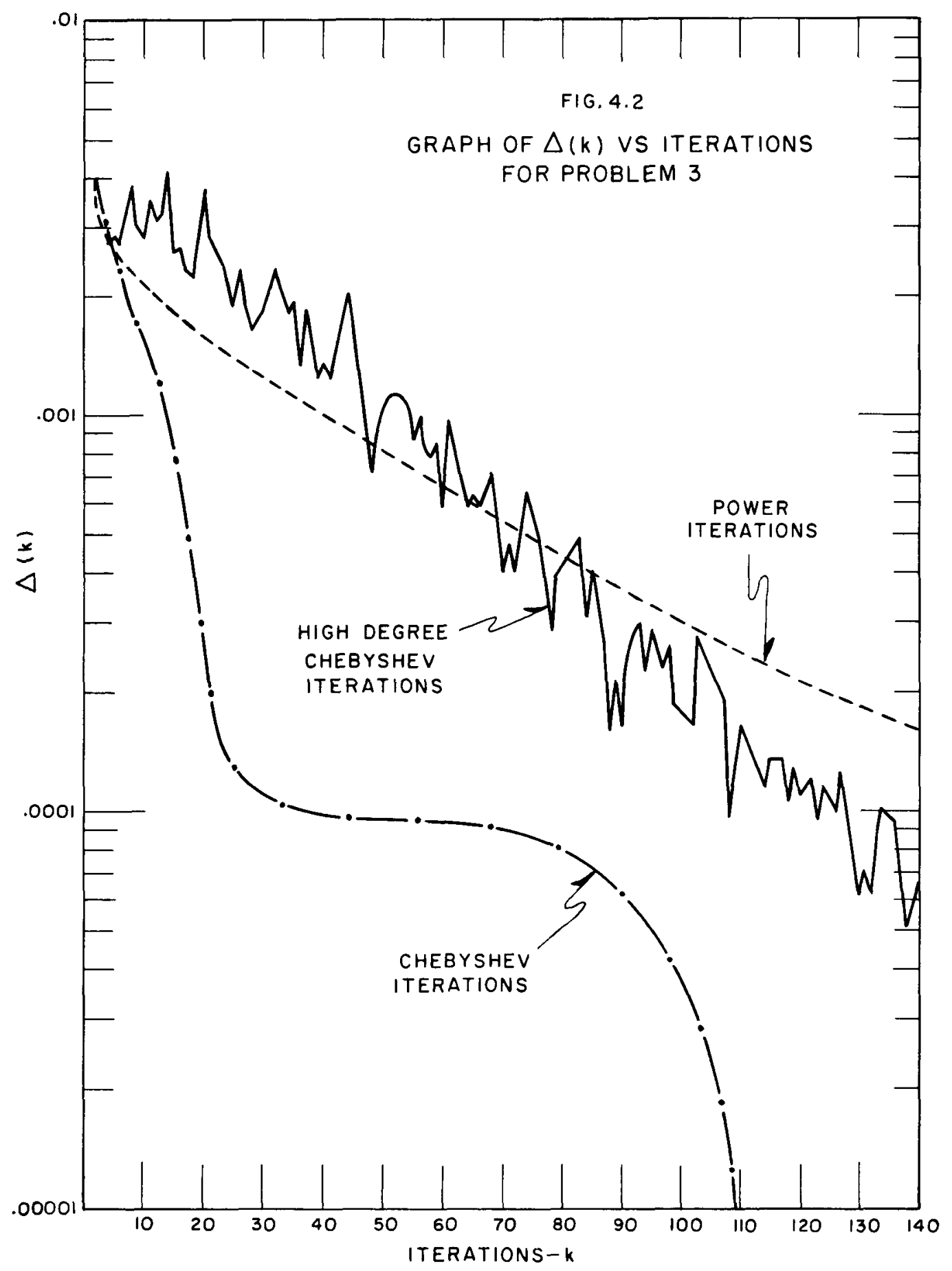


For problem 4, the only fact known concerning the matrix $G$ is that it has a positive dominant eigenvalue. The purpose of this problem is to illustrate the behavior of the complex domain Chebyshev polynomial method described in Chapter II. The numerical results for this problem were obtained from a program designed for a different purpose and, hence, the strategy employed is slightly different and fewer numbers are avaliable as output.

The complex domain Chebysher method requires the use of the three parameters $b, d$, and $\epsilon$ of Figure 2.4. For this problem, $b$ was assumed equal to $-\mathrm{d}$. The problem was run three times using complex chebyshev acceleration: first with fixed $\epsilon=.1$, then with fixed $\epsilon=.5$, and finally with fixed $\epsilon=.75$. In Tables 4.11-4.14, $r$ and $d(k)$ are defined as before. However, $\delta(k)$ is now defined as

$$
\delta(k) \equiv \max _{j}\left|1-\frac{\left[e_{j}, x(k)\right]}{\left[\underline{e}_{j}, \underline{x}(k-2)\right]}\right|
$$

and $Q(k)$ as

$$
Q(k) \equiv \frac{\|\underline{y}(k)\|}{\| \underline{y}(k-2) \underline{H}}
$$

where $\underline{y}(k)=\underline{v}(k)-\underline{x}(k-2)$. The $\delta(k)$ and $Q(k)$ defined above have the same basic meanings as given previously, $i_{0}, ., \delta(k)$ is still a measure of the relative point error and $Q(k)$ can be used to estimate $d$. For power iterations we have $\lim Q(k)=d^{2}$. In the Chebyshev accelerated problems, the estimate $k \rightarrow$ for the dominance ratio is not updated at each iteration. Graphs of $\delta(k)$ vs. $k$ are given in Figures 4.3 and 4.4 . 
Comments on Problem 4:

The $Q(k)$ quantity in Table 4.11 indicates that the matrix G for this problem has rather large complex eigenvalues. To see why this is so, we shall assume that $G$ has some large complex eigenvalues and show that the behavior of $Q(k)$ is the same as that of Table 4.11 .

Let the eigenvalues of $G$ be ordered such thet $\sigma_{1}>\sigma_{2}>\left|\sigma_{3}\right|=$ $\left|\sigma_{4}\right|>\left|\sigma_{5}\right| \geq \ldots$, where $\sigma_{4}=\bar{\sigma}_{3}, i_{0} e_{0}$, we are assuming $\sigma_{3}$ and $\sigma_{4}$ to be complex eigenvalues. Also, let $\underline{x}_{i}$ be the corresponding eigenvectors. Now if $k$ is sufficiently large so that $\underline{x}(k-2)$ can be approximated by $\underline{x}(k-2) \approx \underline{x}_{1}+\underline{x}_{2}+\underline{x}_{3}+\underline{x}_{3}$, then $\underline{y}(k+m)$ can be approximated by

$$
\underline{y}(k+m) \approx d^{m}\left(d^{2}-1\right) \underline{x}_{2}+z^{m}\left(z^{2}-1\right) \underline{x}_{3}+\overline{z^{m}\left(z^{2}-1\right) \underline{x}_{3}}
$$

where $z=\sigma_{3} / \sigma_{1}$. If we denote the $j$-th component of the vector $\left(z^{2}-1\right) \underline{x}_{3}$ by $a_{j} e^{i \beta}$ and the $j-t h$ component of the rector $\left(a^{2}-1\right) x_{2}$ by $x_{j}$, then the $j-t h$ component of $\underline{y}(k+m)$ can be written as

$$
y_{j}(m) \approx d^{m}\left[x_{j}+2 r^{m} a_{j} \cos \left(m \theta+\beta_{j}\right)\right]
$$

where $z \equiv|z| e^{i \theta}$ and $r \equiv|z| / d$. Thus, we have

$$
\|\underline{y}(k+m)\|^{2} \equiv \sum_{j}\left[y_{j}(m)\right]^{2} \approx a^{2 m} \sum_{j}\left[x_{j}^{2}+r^{m_{b}} \cos \left(m \Theta+\beta_{j}\right)\right],
$$

where $b_{j}=4 a_{j} x_{j}$ and where we have assumed the $r^{2 m}$ term in $\left[y_{j}(m)\right]^{2}$ to be negligible. Thus, we have 


$$
[Q(k+m)]^{2} \approx d^{4}\left[\frac{1+r^{m} \sum_{j} e_{j} \cos \left(m \theta+\beta_{j}\right)}{1+r^{m-2} \sum_{j} e_{j} \cos \left((m-2) \theta+\beta_{j}\right)}\right]
$$

where $c_{j}=b_{j} / \sum_{j} x_{j}^{2}$. Again neglecting terms of order grestis than $r$, we have

$$
[Q(k+m)]^{2} \approx d^{4}\left[I+r^{m-2} \sum_{j} c_{j}\left[r^{2}\left(\cos \left(m \theta+\beta_{j}\right)-\cos ((m-2)) \theta+\beta_{j}\right)\right]\right] 。
$$

which may be written as

$$
\left.\left.[Q(k+m)]^{2} \approx a^{4}\left\{1+x^{m-2}[\operatorname{sicos} m \varepsilon)+\sin m \varepsilon\right)\right]\right\}
$$

where $s=\sum_{j} c_{j}\left[r^{2} \cos \beta_{j}-\cos \left(\beta_{j}-2 \theta\right)\right]$ snd where $t=\sum_{j} c_{j}\left[\sin \left(\beta_{j}-2 \theta\right)-r^{2} \sin \beta_{j}\right]$. If $s^{2}+t^{2} \neq 0$, then $s(\cos m \theta)+t(\sin \theta)=v \sin (m \theta+\phi)$, where $v=\left(s^{2}+t^{2}\right)^{1 / 2}$ and $\theta=\sin ^{-1} s / c$. Thus, we have

$$
[Q(k+m)]^{2} \approx a^{4}\left[1+x r^{m-2} \sin (m \theta+\phi)\right] .
$$

Thus, $Q(k+m)$ will oscillate about $d^{2}$ with a period of $2 \pi / \theta$. Since we have assumed $r<1$, the amplitude of this oscillation becomes smaller with m. This is precisely the behavior of $Q(k)$ in Table 4.11 , The pesks in $Q(k)$ have been underlined in Table 4.11

From Table 4.11, we see that the period is between 38 and 42 so that the argument $\theta$ of $z\left(=\sigma_{3} / \sigma_{1}\right)$ is roughly between $8.5^{\circ}$ sind $9.5^{\circ}$. The numerical data of Table 4.11 also implies that the modulus of 2 ia about .93 . Whe Chebysher 
data given in Tables 4.13 and 4.14 imply that the normalized complex eigenvalues of $G$ lie interior to the ellipse

$$
\frac{x^{2}}{(.946)^{2}}+\frac{y^{2}}{(.75)^{2}}=1
$$

but exterior to

$$
\frac{x^{2}}{(.968)^{2}}+\frac{y^{2}}{(.5)^{2}}=1
$$

The complex number $z=.93 e^{i(2 \pi / 40)}$ is interior to both ellipses but $z=.935 \mathrm{e}^{1(2 \pi / 38)}$ is interior to the ellipse of $(4.7)$ and exterior to the ellipse of $(4.8)$. 
PROBLEM 4

POWER ITERATIONS

\begin{tabular}{|c|c|c|c|}
\hline $\mathrm{k}$ & $\mathrm{r}$ & $\delta(\mathrm{k})$ & $Q(\mathrm{k})$ \\
\hline 12 & 0 & .8312430 & .470567 \\
\hline 32 & 0 & .0334180 & 1.287410 \\
\hline 52 & 0 & .0097594 & .783629 \\
\hline 72 & 0 & .0035820 & .997618 \\
\hline 112 & 0 & .0004183 & .937051 \\
\hline 152 & 0 & .0000494 & .914422 \\
\hline 154 & 0 & .0000435 & .914765 \\
\hline 156 & 0 & .0000384 & .913569 \\
\hline 158 & 0 & .0000339 & .911347 \\
\hline 160 & 0 & .0000301 & .908208 \\
\hline 162 & 0 & .0000269 & .904545 \\
\hline 164 & 0 & .0000242 & .900857 \\
\hline 166 & 0 & .0000218 & .897353 \\
\hline 168 & 0 & .0000198 & .894374 \\
\hline 170 & 0 & .0000180 & .892083 \\
\hline 172 & 0 & .0000164 & .890686 \\
\hline 174 & 0 & .0000150 & .890124 \\
\hline 176 & 0 & .0000136 & .890562 \\
\hline 178 & 0 & .0000124 & .891785 \\
\hline 180 & 0 & .0000112 & .893693 \\
\hline 182 & 0 & .0000102 & .895988 \\
\hline 184 & 0 & .0000092 & .898412 \\
\hline 186 & 0 & .0000082 & .900863 \\
\hline 188 & 0 & .0000074 & .902887 \\
\hline 190 & 0 & .0000066 & .904489 \\
\hline 192 & 0 & .0000059 & .905593 \\
\hline 194 & 0 & .0000052 & .905985 \\
\hline 196 & 0 & .0000047 & .9054448 \\
\hline
\end{tabular}


PROBLEM 4

COMPLFX DOMAIN CHFBYSHEV ACCELHRATION - FIXED $\epsilon=: 1$

\begin{tabular}{|c|c|c|c|c|}
\hline$k$ & $r$ & $\delta(k)$ & $Q(k)$ & $d(k)$ \\
\hline 10 & 0 & .954786 & .590 & .768 \\
\hline 18 & 8 & .442435 & .787 & \\
\hline 20 & 0 & .258927 & .593 & .913 \\
\hline 22 & 2 & .146929 & .887 & \\
\hline 38 & 18 & .175314 & .674 & \\
\hline 40 & 0 & .112929 & 1.394 & .900 \\
\hline 42 & 2 & .149251 & .913 & \\
\hline 44 & 4 & .148386 & 1.005 & \\
\hline 46 & 6 & .286167 & 2.155 & \\
\hline 48 & 8 & .485156 & 1.300 & \\
\hline 50 & 10 & $=971887$ & .843 & \\
\hline 52 & 12 & 1.617660 & 1.986 & \\
\hline 54 & 14 & $4 \cdot 354200$ & .713 & \\
\hline 56 & 16 & 3.90364 & 1.587 & \\
\hline 58 & 18 & 84.09410 & 1.317 & \\
\hline 60 & 20 & 54.00010 & .893 & \\
\hline 62 & 22 & $22 \cdot 94770$ & 1.539 & \\
\hline 64 & 24 & $27 \cdot 97570$ & 1.139 & \\
\hline 66 & 26 & $18 \cdot 38360$ & .602 & \\
\hline 68 & 28 & 85.22920 & 1.727 & \\
\hline 70 & 0 & 16.95400 & .903 & .900 \\
\hline 72 & 2 & $75 \cdot 16020$ & .833 & \\
\hline 100 & 30 & 53.60490 & 2.095 & \\
\hline
\end{tabular}

TABLE 4.12 
FROBLEM 4

COMPLEX DOMAIN CHEBYSHEV ACCELERATION - FIXED $\approx=.5$

\begin{tabular}{|c|c|c|c|c|}
\hline $\mathbf{k}$ & $r$ & $\delta(k)$ & $Q(k)$ & $d(k)$ \\
\hline 10 & 0 & .954786 & .590 & .768 \\
\hline 18 & 8 & .427459 & .809 & \\
\hline 20 & 0 & .290486 & .738 & .906 \\
\hline 22 & 2 & .207921 & $.86 ?$ & \\
\hline 38 & 18 & .022285 & .541 & \\
\hline 40 & 0 & .018444 & .347 & .967 \\
\hline 42 & 2 & .019464 & .549 & \\
\hline 44 & 4 & .025491 & 1.405 & \\
\hline 46 & 6 & .029178 & 1.116 & \\
\hline 48 & 8 & .028033 & .890 & \\
\hline 50 & 10 & .017541 & .479 & \\
\hline 52 & 12 & .012640 & 1.328 & \\
\hline 54 & 14 & .011752 & 1.601 & \\
\hline 56 & 16 & .006478 & 1.204 & \\
\hline 58 & 18 & .012880 & 1.221 & \\
\hline 60 & 20 & .009910 & .972 & \\
\hline 62 & 22 & .008962 & .404 & \\
\hline 64 & 24 & .007952 & 1.084 & \\
\hline 66 & 26 & .008748 & 1.592 & \\
\hline 68 & 28 & .006889 & .309 & \\
\hline 70 & 0 & .007033 & 2.764 & .900 \\
\hline 72 & 2 & .004470 & .886 & \\
\hline 108 & 38 & .001312 & 2.097 & \\
\hline 110 & 0 & .000825 & .594 & .968 \\
\hline 112 & 2 & .000701 & .768 & \\
\hline 158 & 48 & .006667 & .854 & \\
\hline 198 & 38 & .001256 & .451 & \\
\hline
\end{tabular}


PROBLEM 4

COMPLEX DOMAIN CHEBYSHEV ACCELERATION - FIXED $\epsilon=.75$

\begin{tabular}{|c|c|c|c|c|}
\hline $\mathrm{k}$ & $r$ & $\delta(k)$ & $Q(k)$ & $d(k)$ \\
\hline 10 & 0 & .9547860 & .590 & .768 \\
\hline 18 & 8 & .4150640 & .827 & \\
\hline 20 & 0 & $\cdot 3155640$ & .844 & .899 \\
\hline 22 & 2 & .2434230 & .864 & \\
\hline 38 & 18 & .0231445 & .973 & \\
\hline 40 & 0 & .0193948 & .823 & .900 \\
\hline 42 & 2 & .0156790 & .840 & \\
\hline 44 & 4 & .0154278 & .845 & \\
\hline 46 & 6 & .0128705 & .731 & \\
\hline 48 & 8 & .0118865 & .730 & \\
\hline 50 & 10 & .0120340 & .741 & \\
\hline 52 & 12 & .0128080 & .768 & \\
\hline 54 & 14 & .0127994 & .803 & \\
\hline 56 & 16 & .0119710 & .841 & \\
\hline 58 & 18 & .0103050 & .885 & \\
\hline 60 & 20 & .0082678 & .934 & \\
\hline 62 & 22 & .0063066 & .984 & \\
\hline 64 & 24 & .0046881 & 1.019 & \\
\hline 66 & 26 & .0034826 & 1.030 & \\
\hline 68 & 28 & .0026561 & 1.014 & \\
\hline 70 & 0 & .0020136 & .911 & .941 \\
\hline 72 & 2 & .0019455 & .947 & \\
\hline 108 & 38 & .0002086 & .870 & \\
\hline 110 & 0 & .0001729 & .766 & .946 \\
\hline 112 & 2 & .0001714 & .865 & \\
\hline 158 & 48 & .0000110 & .886 & \\
\hline 160 & 0 & .0000085 & .803 & .946 \\
\hline
\end{tabular}

TABLE 4.14 


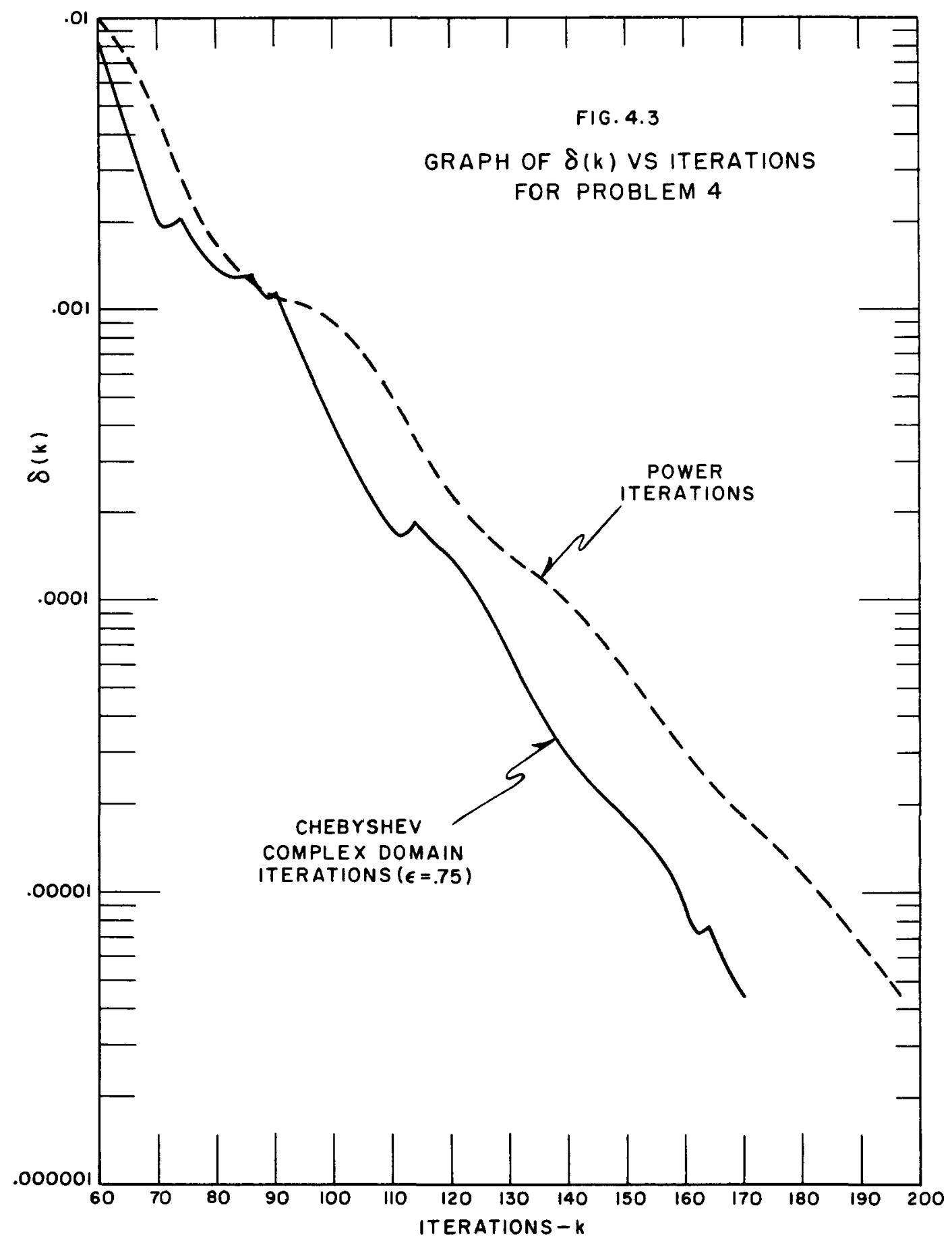




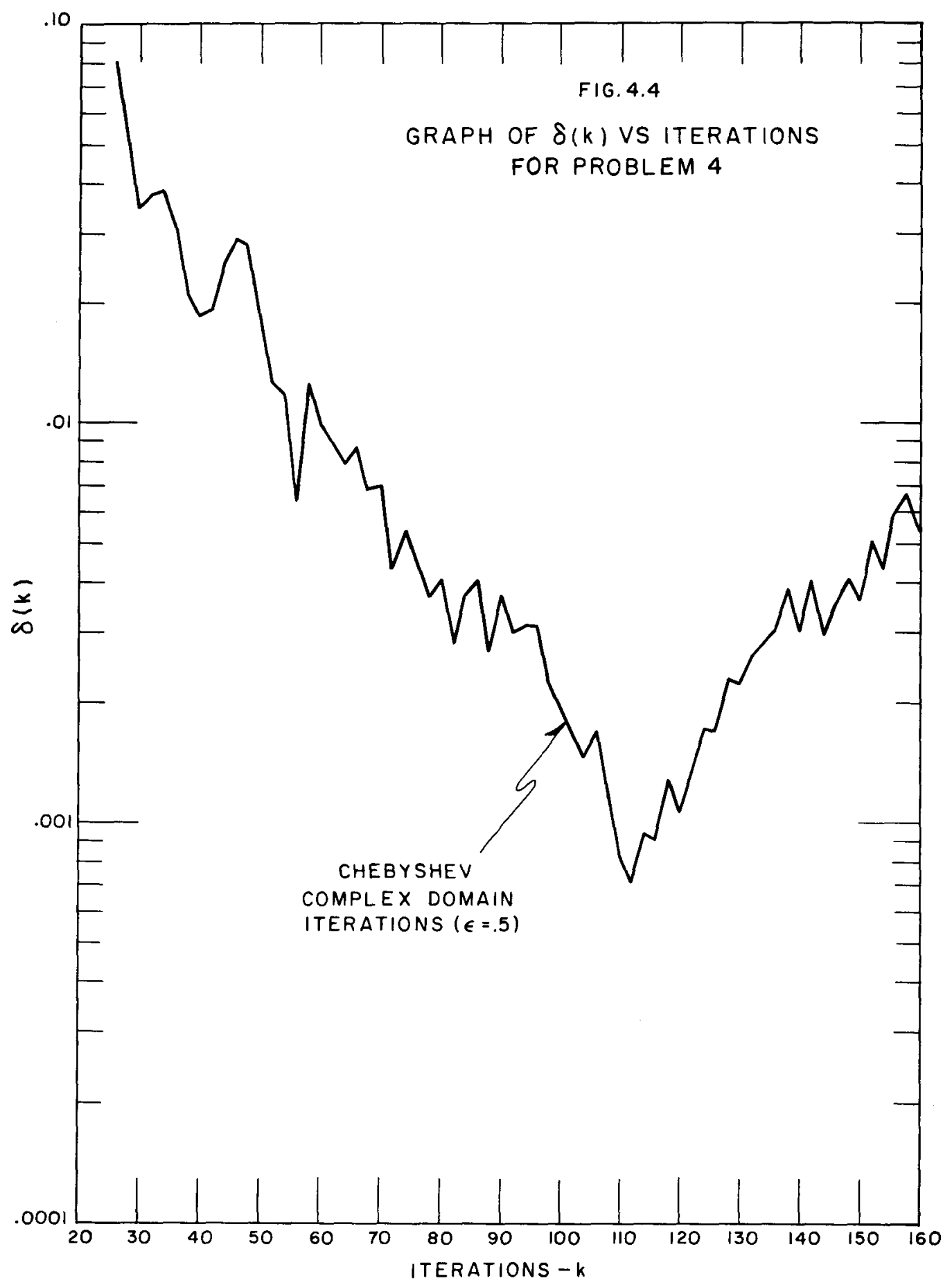


THE REAL DOMAIN CHEBYSHEV POLYNOMIAL

AND COMPLEX EIGENVALUES

In this appendix, we wish to find the set of points, $D_{r}(c)$, in the complex $z$ plane which satisfy the inequality

$$
\left|P_{r, d_{O}}(z)\right| \leq\left\{c F_{r}\right\}^{r}
$$

where $1 \leq c \leq 1 / F_{r}$,

$$
P_{r, d_{0}}(z)=\frac{T_{r}\left(\frac{2 z-d_{0}-b_{0}}{d_{0}-b_{0}}\right)}{T_{r}\left(\frac{2-d_{0}-b_{0}}{d_{0}-b_{0}}\right)},
$$

and

$$
F_{r}=\left\{\frac{1}{T_{r}\left(\frac{2-d_{0}-b_{0}}{d_{0}-b_{0}}\right)}\right\}^{1 / r}
$$

From $(A .2)$ and $(A .3)$, the set of points $D_{r}(C)$ may also be expressed as

$$
D_{r}(c) \equiv\left\{z:\left|T_{r}\right| \frac{2 z-d_{0}-b_{0}}{d_{0}-b_{0}} \mid \leq c^{r}\right\}
$$

We shall consider the special cases of $r=1,2$ and the limit as $r$ approaches $\infty$.

CASE 1: $r=1$

Since $T_{1}(s)=s$, we seek those $z$ which satisfy the inequality

$\left|\frac{2 z-a_{0}-b_{0}}{d_{0}-b_{0}}\right| \leq c$. Since $b_{0}<d_{0}, d_{0}-b_{0}>0$ and hence we may write 
(A.5)

$$
\left|z-\left(\frac{a_{0}+b_{0}}{2}\right)\right| \leq c\left(\frac{a_{0}-b_{0}}{2}\right)
$$

Thus, the set $D_{1}(c)$ consists of all $z$ on or interior to the circle with center at $\left(0, \frac{a_{0}+b_{0}}{2}\right)$ and radius $c\left(\frac{d_{0}-b_{0}}{2}\right)$. Using the maximum modulus theorem, one may easily show that no $z$ exterior to this circle satisfies (A.5).

CASE 2: $r=$ ?

For this case $T_{2}(s)=2 s^{2}-I$ and thus we seek those $z$ which satisfy

$$
\left.|2| \frac{2 z-a_{0}-b_{0}}{a_{0}-b_{0}}\right|^{2}-1 \mid \leq c^{2}
$$

If we let $a=\frac{d_{0}-b_{0}}{2 \sqrt{2}}$, then we may write $(A .6)$ as

$$
\mid \frac{1}{a^{2}}\left(z-\left.\left(\frac{a_{0}+b_{0}}{2}\right)\right|^{2}-a^{2} \mid \leq c^{2}\right.
$$

or equivalently

$$
\left|\left\{z-\left|\frac{a_{0}+b_{0}}{2}\right|+a\right\}\left\{z-\left|\frac{a_{0}+b_{0}}{2}\right|-a\right\}\right| \leq(a c)^{2} .
$$

If $z=x+i y$, then (A.7) becomes

(A.8)

$$
\sqrt{\left\{\left[x-\left(\frac{a_{0}+b_{0}}{2}\right)\right]+a\right\}^{2}+y^{2}} \sqrt{\left\{\left[x-\left(\frac{a_{0}+b_{0}}{2}\right)\right]-a\right\}^{2}+y^{2}} \leq(a c)^{2}
$$

The points $(x, y)$ satisfying the equality in (A.8) lie on the ovals of Cassini. See Figure $A .1$. 


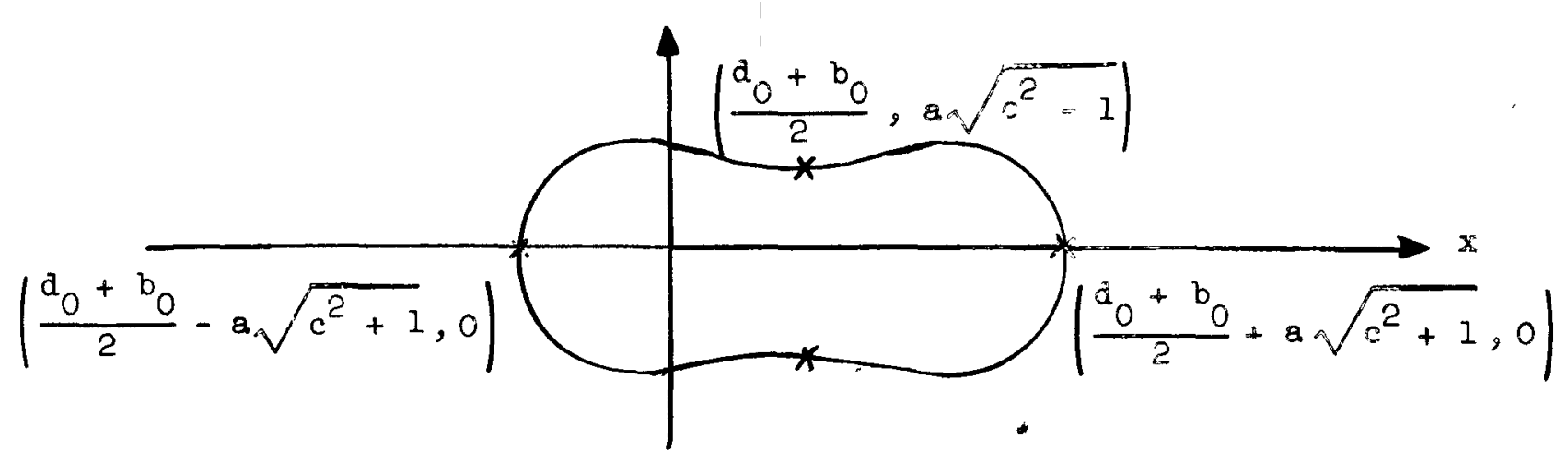

Figure A.1

Thus, again using the maximum modulus theorem, the set. $7_{2}(0)$ consists of all $z$ on or interior to the ovals of Cassini.

CASE 3: $r \rightarrow \infty$

If we let

(A.9)

$$
s=\frac{z-\left(\frac{a_{0}+b_{0}}{2}\right)}{\frac{a_{0}-b_{0}}{2}},
$$

then the Chebyshev polynomial $\operatorname{T}_{r}\left(\frac{2 z-d_{0}-b_{0}}{d_{0}-b_{0}}\right)$ may be expressed [Forsythe and Wasow (1960), p. 228] as

$$
T_{r}(s)=\frac{1}{2}\left(\left(s+\sqrt{s^{2}-1}\right)^{r}+\left(s-\sqrt{s^{2}-1}\right)^{r}\right\}
$$

or equivalently as

$$
T_{r}(s)=\frac{1}{2}\left(\left(s+\sqrt{s^{2}-1}\right)^{r}+\left(s+\sqrt{s}-1^{-r}\right) .\right.
$$


Now let

$$
w=s+\sqrt{s^{2}-1}
$$

Thus, $\left|T_{r}(s)\right| \leq c^{r}$ when $\left|1 / 2\left[w^{r}+w^{-r}\right]\right| \leq c^{r}$ or equivalently when

$$
\left|\left(\frac{w}{c}\right)^{r}+\frac{1}{(w c)^{r}}\right| \leq 2 .
$$

As $r$ approaches $\infty$, the inequality (A.12) is satisfied if and only if ${ }^{l}$

$$
\left|\frac{w}{c}\right| \leq 1 \text { and }\left|\frac{1}{w c}\right| \leq 1
$$

or equivalently if and only if

$$
|w| \leq c \text { and }|w| \geq=1 / c \text {. }
$$

Hence, $w$ must lie in the closed annulus between the circles $|w|=1 / c$ and $|w|=c$. We now shall proceed to get the corresponding $z$ region. Solving Eq. (A.Il) for $s$ gives

$$
s=1 / 2[w+1 / w) .
$$

If $c>1$, then the annulus

The if part is obvious. To show the only if part, one need only show that if $\frac{w}{c}$ or $1 /|w c|$ is greater than unity, then

$$
\lim _{r \rightarrow \infty}\left|\left(\frac{w}{c}\right)^{r}+\left(\frac{l}{w c}\right)^{r}\right|=\infty \text {. }
$$




$$
1 / c \leq|w| \leq c
$$

in the w plane is mapped [Kober (1957), pg。62] onto the closed region

$$
|s-1|+|s+1| \leq \frac{c+1 / c}{2}
$$

in the s plane and onto the closed region

$$
\left|z-a_{0}\right|+\left|z-b_{0}\right| \leq \frac{\left(a_{0}-b_{0}\right)\left(c+\frac{1}{c}\right)}{4}
$$

in the $z$ plane. See Figure A.2. If $z=x+1 y$, then the closed region described by the inequality $(A .15)$ is simply the ellipse

$$
\frac{\left[x-\frac{a_{0}+b_{0}}{2}\right]^{2}}{\left[\left(\frac{d_{0}-b_{0}}{4}\right)\left(c+\frac{1}{c}\right)\right]^{2}}+\frac{y^{2}}{\left[\left(\frac{a_{0}-b_{0}}{4}\right)\left(c-\frac{1}{c}\right)\right]^{2}}=1
$$

and its interior.

If $c=1$, the circle $|w|=1$ is mapped onto the line segment

$-I \leq s \leq 1$ in the $s$ plane and onto the line segment $b_{0} \leq z \leq d_{0}$ in the $z$ plane. Thus, in the limit as $r$ approaches infinity, the region $D_{\infty}(c)$ consists $^{1}$ of all $z$ on or interior to the ellipse (A.16). The regions $D_{1}(c), D_{2}(c)$, and $D_{\infty}(c)$ for $c=1$ and $c=1 / F_{r}$ are given in Figures $2.1,2.2$ and 2.3.

$\overline{\mathrm{I}_{A} \text { similar type }}$ result is given by Wrigley (1963). 

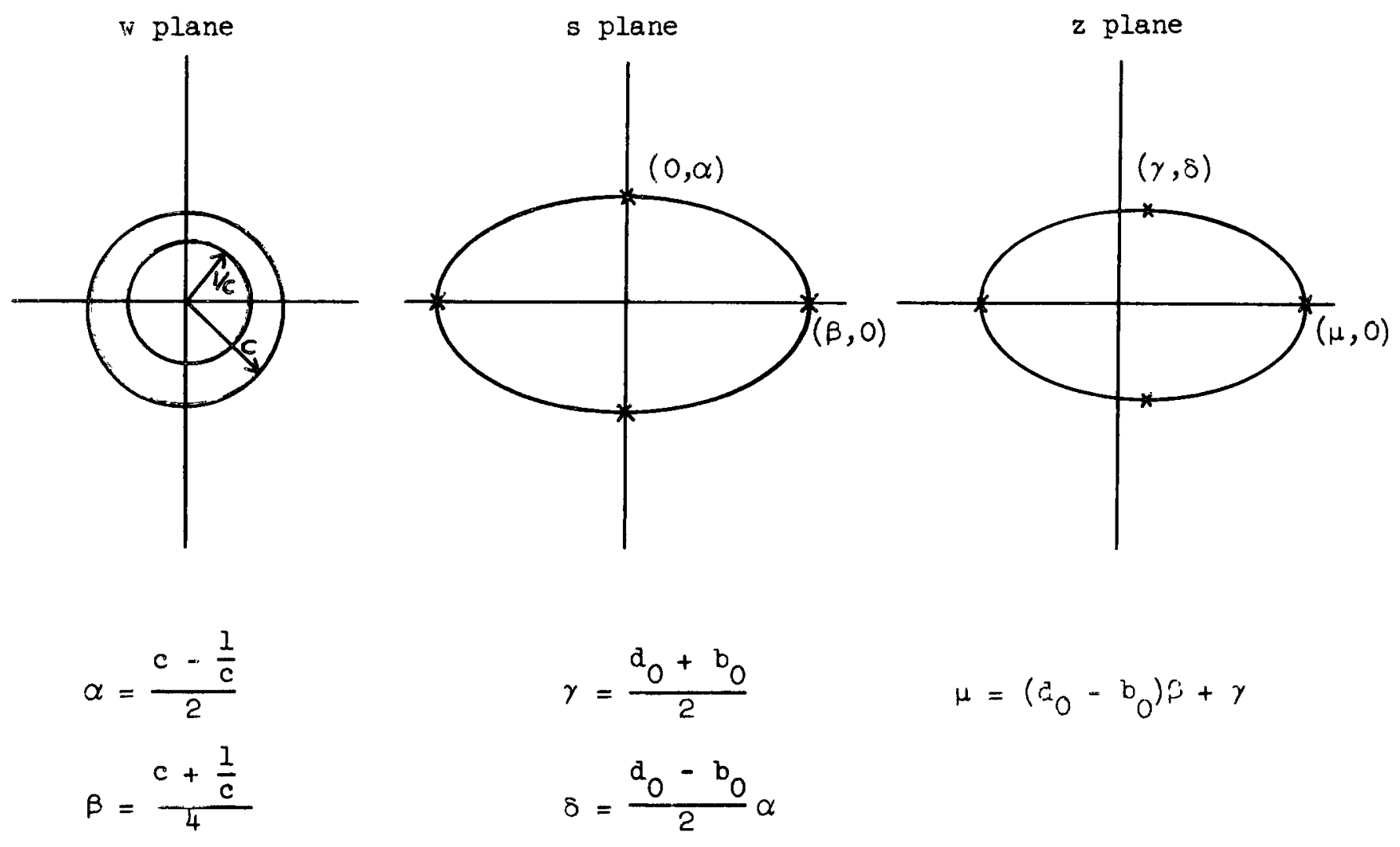

Figure A.2 
In this appendix we shall describe briefly the use of the Chebyshev polynomial method of iteration in the solution of the inhomogeneous matrix problem

$$
A \underline{x}=\underline{s},
$$

where, for convenience, we assume that the nxn nonsingular matrix $A$ is girs by $A=I-B$. We shall also assume that the eigenvalues $\left\{\sigma_{i}\right\}_{i=1}^{i=n}$ of $I$ are real and are ordered such that

$$
\left|\sigma_{n}\right| \leq\left|\sigma_{n=1}\right| \leq \cdots \leq\left|\sigma_{2}\right|<\sigma_{1}<1
$$

and that the set of eigenvectors for $B$ span the associated vector space.

The matrix problem (B.I) may be solved iteratively using the well known Jacobi method

$$
\underline{x}(k+1)=\operatorname{Bx}(k)+\underline{s},
$$

where $k$ is the iteration index number. If $\underline{E}(k) \equiv \underline{x}(k)-\underline{x}$ is the error vector after $k$ iterations, then it follows from (B.I) and (B.3) that

$$
\underline{E}(k)=\underline{B E}(k-1)=B^{k} \underline{E}(0)
$$

Expanding $E(0)$ in terms of the eigenvectors of $B$ gives

$$
E(k)=B^{k}\left[\sum_{i} c_{i} \underline{x}_{i}\right]=\sum_{i}\left(\sigma_{i}\right)^{k} c_{i=i} .
$$


Hence, for the Jacobl method of iteration, we see that the most slowly decaying contribution to the intial error vector goes to zero as $\left(\sigma_{1}\right)$.

To solve (B.I) using the Chebyshev polynomial method of iteration, one can use [Varga (1962), pg. 138] the iterative procedure

$$
\underline{x}(k+1)=w_{k+1}[\underline{v}(k+1)-\underline{x}(k-1)]+\underline{x}(k-1),
$$

where $\underline{v}(k+1)=\underline{B} \underline{x}(k)+\underline{s}$. The sequence $w_{k+1}$ is given by $\omega_{1}=1$ and

$$
\omega_{k+1}=\frac{2 T_{k}\left(\frac{1}{\sigma_{1}}\right)}{\sigma_{1} T_{k+1}\left(\frac{1}{\sigma_{1}}\right)}, k \geq 1
$$

where $T_{k}(w)$ is the Chebyshev polynomial of degree $k$ in $w$. The error vector $\mathrm{E}^{(\mathrm{k})}$ after $\mathrm{k}$ Chebyshev Iterations can be [Varga (1962)] given by

$$
\underline{E}(k)=P_{k}(B) E(0),
$$

where

$$
P_{k}(w) \equiv \frac{T_{k}\left(\frac{w}{\sigma_{1}}\right)}{T_{k}\left(\frac{1}{\sigma_{1}}\right)}
$$

Thus, the expansion of $\underline{E}(0)$ gives

$$
E(k)=P_{k}(B)\left[\sum_{1} c_{i-1}\right]=\sum_{i} P_{k}\left(\sigma_{i}\right) c_{i-1} .
$$

Hence, we see that the most slowly decaying contribution to the initial error vector for the Chebyshev iterations goes to zero as $P_{k}\left(\sigma_{1}\right)$. 
As for the eigenvalue problem, the rate of convergence of the Cheby shev iterations (B.4) is greatly affected by the estimate for $\sigma_{1}$. In practice, two basic approaches are often used to estimate $\sigma_{1}$. One approach [see, for example, Varga (1962), Wachspress (1966), Forsythe and Wasow (1960), and Hageman and Kellogg (1966)] is to obtain an estimate for $\sigma_{1}$ prior to carrying out the Chebysher 1terations. For example, an a priori estimate for $\sigma_{1}$ may be obtalned by using the power or Chebyshev iteration method on the matrix $B$. The second approach is to obtain estimates for $\sigma_{1}$ while carrying out the Chebyshey iter ations. In what follows we shall describe a Chebysher strategy, similar to thet given for the eigenvalue problem, which may be used to obtain estimates for $\sigma_{1}$ while carrying out the Chebysher iterations.

As before, numerical estimates for $\sigma_{1}$ will be obtained by observing the decay rate of the residual vector $\underline{y}(k) \equiv \underline{v}(k)-\underline{x}(k-1)$, where $\underline{v}(k)=B \underline{x}(k-1)+\underline{s}$. We w11l again use the residual vector quotient

$$
Q(k) \equiv \frac{\|\underline{y}(k)\|}{\|\underline{y}(k-1)\|}
$$

to measure the decay rate and the same Chebyshev strategy as described in Chapter II in obtaining estimates for $\sigma_{1}$.

An initial estimate for $\sigma_{1}$ can be obtained by doing a few Jacobi iterations before starting the Chebyshev iterations. For the Jacobi method. we have $\lim Q(k)=\sigma_{1}$. This follows from the fact that the resianal vectors for the Jacobl method satisfy

$$
\underline{y}(k)=B \underline{y}(k-I)=B^{k-1} \underline{y}(1) .
$$


Suppose now that a Chebyshev polynomial using $d_{0}$ as the estimate for $\sigma_{1}$ is started on iteration $k_{1}+1$. Then for iteration $k_{1}+r+1$, $\underline{\mathrm{y}}\left(\mathrm{k}_{1}+\mathrm{r}+\mathrm{l}\right)=(\mathrm{B}-\mathrm{I}) \underline{\mathrm{x}}\left(\mathrm{k}_{1}+\mathrm{r}\right)+\underline{\mathrm{s}}$ and since $\underline{\mathrm{s}}=(I-B) \underline{\mathrm{x}}$ we have

$$
\underline{y}\left(k_{1}+r+1\right)=(B-I) \underline{E}\left(\underline{k}_{1}+r\right) .
$$

Using (B.5) we then get

$$
\underline{y}\left(k_{1}+r+1\right)=(B-I) P_{r, d_{0}}(B) \underline{E}\left(k_{1}\right) \text {. }
$$

But from $(B \cdot 7), \underline{E}\left(k_{1}\right)=(B-I)^{-1} \underline{y}\left(k_{1}+1\right)$ and since $B$ - I commutes with $\mathrm{P}_{\mathrm{r}, \mathrm{d}_{0}}(\mathrm{~B})$ we have

$$
\underline{\mathrm{y}}\left(\mathrm{k}_{1}+r+1\right)=P_{r, \mathrm{~d}_{0}}(B) \underline{\mathrm{y}}\left(\mathrm{k}_{1}+1\right) \text {. }
$$

Thus, for $\mathrm{k}_{1}$ large enough we have

$$
Q\left(k_{1}+r+1\right) \approx\left|\frac{P_{r, d_{0}}\left(\sigma_{1}\right)}{P_{r-1, d_{0}}\left(\sigma_{1}\right)}\right|
$$

and

$$
Q_{r+1} \approx\left|P_{r, d_{0}}\left(\sigma_{1}\right)\right|
$$

where $Q_{r+1}=\prod_{l=2}^{r+1} Q_{\left(k_{1}+l\right)}$. Note that Eqs. $(B \cdot 9)$ and $(B \cdot 10)$ are the same as those obtained for the elgenvalue problem. Thus, as before, one may obtain a new estimate for $\sigma_{1}$ by solving Eq. (B.10) for $\sigma_{1}$. 
If the eigenvalues of $B$ are real but now satisfy

$$
\left|\sigma_{n}\right| \leq\left|\sigma_{n-1}\right| \leq \cdots \leq\left|\sigma_{3}\right|<\left|\sigma_{2}\right|=\sigma_{1}<1
$$

where $\sigma_{2}=-\sigma_{1}$, then the above procedure can still be used provided the following changes are noted.

First redefine $Q(k)$ as

$$
Q(2 k) \equiv \frac{\|\underline{y}(2 k)\|}{\|\underline{\underline{y}}(2(k-1))\|}
$$

Then for the Jacobi iterations we have $\lim _{k \rightarrow \infty} Q(2 k)=\left(\sigma_{1}\right)^{2}$ and in place of (B.9) we have

$$
Q\left(k_{1}+2 r+1\right) \approx\left|\frac{P_{2 r, d_{0}}\left(\sigma_{1}\right)}{P_{2(r-1), d_{0}\left(\sigma_{1}\right)}}\right| .
$$

Equation (B.10) should be replaced by

$$
\frac{\| \underline{y}\left(k_{1}+2 r+1\|\|\right.}{\left\|\underline{y}\left(k_{1}+1\right)\right\|} \approx\left|P_{2 r, d_{0}}\left(\sigma_{1}\right)\right| .
$$

In the next section we shall consider the special cyclic Chebysher polynomial method.

\section{The Cyclic Chebysher Method}

In this section we assume that the matrix $B$ is written in the special cyclic form $B=\left(\begin{array}{ll}0 & U \\ L & 0\end{array}\right)$ and that Eq. (B.I) is written in form 
(B.Il)

$$
\left(\begin{array}{cc}
I & -U \\
-I & I
\end{array}\right)\left(\begin{array}{l}
\underline{x}_{1} \\
\underline{x}_{2}
\end{array}\right)=\left(\begin{array}{l}
\underline{s}_{1} \\
\underline{s}_{2}
\end{array}\right) \text {. }
$$

The cyclic Chebyshev method of iteration is then defined [Varga (1962), pg. 150] to be

$$
\left\{\begin{array}{l}
\underline{x}_{1}(k+1)=\alpha_{k+1}\left[\underline{v}_{1}(k+1)-\underline{x}_{-1}(k)\right]+\underline{x}_{1}(k), \text { where } \underline{v}_{1}(k+1)=U \underline{x}_{-2}(k)+\underline{s}_{1} \\
\underline{x}_{-2}(k+1)=\beta_{k+1}\left[\underline{v}_{2}(k+1)-\underline{x}_{2}(k)\right]+\underline{x}_{2}(k), \text { where } \underline{v}_{-2}(k+1)=\underline{L} \underline{x}_{1}(k+1)+\underline{s}_{2}
\end{array}\right.
$$

The sequences $\alpha_{k+1}$ and $\beta_{k+1}$ are given by $\alpha_{1}=1, \beta_{1}=2 /\left(2-\sigma_{1}^{2}\right)$ and for $k \geq 1$

$$
\alpha_{k+1}=\frac{1}{1-\frac{\sigma_{1}^{2}}{4} \beta_{k}}, \quad \beta_{k+1}=\frac{1}{1-\frac{\sigma_{1}^{2}}{4} \alpha_{k+1}} \text {. }
$$

It can be shown [Varga (1962)] that the error vectors $\underline{E}_{1}(k) \equiv \underline{x}_{1}(k)-\underline{x}_{1}$ and $\underline{E}_{2}(k) \equiv \underline{x}_{2}(k)-\underline{x}_{2}$ satisfy the equations

$$
E_{-1}(k)=S_{k-1}(U L) U E_{2}(0), \underline{E}_{2}(k)=R_{k}(I U) \underline{E}_{2}(0),
$$

where $S_{k}(U L)$ and $R_{k}$ (LU) are polynomials of degree $k$ in $U L$ and LU, respectively, and where $S_{0}(U L)=R_{0}(I U)=I$. Also

$$
R_{k}\left(w^{2}\right)=P_{2 k}(w) \text { and } w S_{k}\left(w^{2}\right)=P_{2 k+1}(w)
$$

where $P_{k}(w)$ is given by $(B .5)$.

$$
\text { Since } \underline{s}_{2}=-I \underline{x}_{1}+\underline{x}_{2} \text {, the residual vector } \underline{y}_{2}(k+1) \equiv \underline{v}_{2}(k+1)-\underline{x}_{2}(k)
$$

can be expressed as 


$$
\underline{y}_{2}(k+1)=\underline{L x}_{-1}(k+1)-I x_{1}+x_{2}-\underline{x}_{2}(k)=L \underline{I E}_{1}(k+1)-\underline{E}_{2}(k)
$$

and using (B.13) we get

$$
\underline{y}_{2}(k+I)=\left[L S_{k}(U L) U-R_{k}(L U)\right] \underline{E}_{2}(0)
$$

or equivalently since $\underline{E}_{2}(0)=(L U-I)^{-1} \underline{y}_{2}(1)$

$$
\underline{y}_{2}(k+1)=\left[L S_{k}(U L) U-R_{k}(L U)\right][L U-I]^{-1} \underline{y}_{2}(1)
$$

This expression is not quite as nice as the corresponding expression (B.8) for the normal Chebyshev method. However, if $\alpha_{0}$ is the estimate for $\sigma_{1}$ used in the generation of the $\alpha$ and $\beta$ sequences and if $\alpha_{0}<\sigma_{1}$, then for $k$ sufficiently large we still have

$$
Q(k+1) \approx\left|\frac{P_{2 k, d_{0}}\left(\sigma_{1}\right)}{P_{2 k-2, d_{0}}\left(\sigma_{1}\right)}\right|,
$$

where $Q(k+1) \equiv\left\|\underline{y}_{2}(k+1)\right\| /\left\|\underline{y}_{2}(k)\right\|$. But now

$$
Q_{k+1} \equiv \frac{\left\|\underline{y}_{2}(k+1)\right\|}{\left\|\underline{y}_{2}(1)\right\|} \approx f\left(\sigma_{1}, d_{0}\right) P_{2 k, d_{0}}\left(\sigma_{1}\right)
$$

where $f\left(\sigma_{1}, d_{0}\right)$ is some function in $\sigma_{1}$ and $d_{0}$.

An alternate approach is the following: Let the error vector $\mathrm{E}_{2}\left(\mathrm{k}_{1}\right)$ at the end of $k_{1}$ iterations be given by (B.13), i.e., $\underline{E}_{2}\left(k_{1}\right)=R_{k_{1}}(L U) E_{2}(0)$. Now suppose on Iteration $k_{1}+1$, a new Chebysher polynomial is started, i.e., the $\alpha$ and $\beta$ sequences are started over in (B.12). Then from (B.15) we have 


$$
\underline{\mathrm{y}}_{2}\left(\mathrm{k}_{1}+\mathrm{I}\right)=(\mathrm{LU}-\mathrm{I}) \underline{\mathrm{E}}_{2}\left(\mathrm{k}_{1}\right)=(L \mathrm{U}-\mathrm{I}) \mathrm{R}_{\mathrm{k}_{1}}(\mathrm{LU}) \underline{\mathrm{E}}_{2}(0)
$$

But $\underline{E}_{2}(0)=(I U-I)^{-1} \underline{y}_{2}(I)$ so that

$$
\underline{y}_{2}\left(k_{1}+1\right)=R_{k_{1}}(L U) \underline{y}_{2}(I)
$$

Hence, under suitable conditions we have

$$
\frac{\left\|\underline{y}_{2}\left(k_{1}+1\right)\right\|}{\left\|\underline{y}_{2}(1)\right\|} \approx P_{2 k_{1}, d_{0}}\left(\sigma_{1}\right)
$$

The alternate approach has the disadvantage that the generation of the Chebyshev polynomial must be terminated in order to obtain a new estimate for $\sigma_{1}$. However, the effect of this should be small if proper care is taken. 


\section{REFERENCES}

1. Bilodeau, G. G. and Hageman, L. A., (1957), "A Survey of Numerical Methods in the Solution of Diffusion Problems, WAPD-TM-64.

2. Clayton, A. J., (1963), "Further Results on Polynomials Having Least Maximum Modulus Over an Ellipse in the Complex Plane, U.K.A.E.A. Memorandum -AEEW- M. 348.

3. Faddeev, D. K. and Faddeeva, V. No, (1963), Computat1onsl Methods of Linear Algebra, W. H. Freeman and Company, San Francisco and London.

4. Flanders, D. A. and Shortley, G., (1950), "Numerical Determination of Fundamental Modes," J.Appl. Physics, Vol. 2l, pages 1326-1332.

5. Forsythe, G. E. and Wasow, W. R., (1960), Finite Difference Methods for Partial Differential Equations, John Wiley and Sons, Inc., New York and London.

6. Hageman, L. A., (1963), "Numerical Methods and Techniques Used in tbe Two-Dimensional Neutron-Diffusion Program PDQ $-58^{81}$ WAPD-TM=364。

7. Hageman, L. A. and Kellogg, R. B. (1966), "Estimating Optimum Overo relaxation Parameters," WAPD-MM-592.

8. Halmos, P. R., (1957), Introduction to Hilbert Space, Chelsea Publishing Company, New York。

9. Householder, A. S. (1953), Principles of Numerical Analysis, McGraw-Hill Book Co., Inc., New York。

10. Kober, H. (1957), Dictionary of Conformal Representations, Dover Publications, Inc.

11. National Bureau of Standards Applied Mathematics Series .9 (1952), Tables of Chebysher Polynomials $S_{n}(x)$ and $c_{n}(x)$.

12. Perlis, So, (1952), Theory of Matrices, Addiscn-Wesley Press, Inc。 Cambridge.

13. Varga, R。 So, (1957), "On Estimating Rates of Convergence in Multigroup Diffusion Problems," WAPD-TM-4I.

14. Varga, Ro S. (1962), Matrix Iterative Analysis, PrenticemHall, Inc。 Englewood Cliffs, New Jersey.

15. Wachspress, E. L。, (1966), Iterative Solution of Elliptic Systems, Prentice-Hall, Inc., Englewood Cliffs, New Jersey.

16. Wrigley, H. E., (1963), "Accelerating the Jacobi Method for Solving Simul= taneous Equations by Chebyshev Extrapolation when the Eigenvalues of the Iteration Matrix are Complex," Computer Journal, Vol。6, pages 169-176. 


\section{ACKNOWLEDGEMENT}

The author would like to thank $\mathrm{Dr} . \mathrm{R}$. B. Kellogg for his many comments and suggestions concerning the contents of this paper. 